\title{
Differentiating Catalysis in the Dearomative [4+2]-Cycloaddition Involving Enals and Heteroaromatic Aldehydes
}

\author{
Aleksandra Topolska, ${ }^{a}$ Sebastian Frankowski, ${ }^{a}$ and Lukasz Albrecht ${ }^{\mathrm{a}^{*}}$ \\ ${ }^{a}$ Institute of Organic Chemistry \\ Faculty of Chemistry \\ Lodz University of Technology \\ Żeromskiego 116, 90-924 Łódź, Poland \\ E-mail: lukasz.albrecht@p.lodz.pl
}

\section{Contents}

$\begin{array}{ll}\text { 1. General methods } & \text { S2 }\end{array}$

2. Differentiating catalysis in the [4+2]-cycloaddition - general procedure S3

3. Selective transformations of the product 3a $\quad$ S10

4. Crystal and X-ray data for 3a $\quad$ S13

5. Non-linear effects study $\quad$ S16

$\begin{array}{ll}\text { 6. NMR data } & \text { S17 }\end{array}$

$\begin{array}{lll}\text { 7. } \mathrm{UPC}^{2} \text { data } & \text { S35 }\end{array}$ 


\section{General methods}

NMR spectra were acquired on a Bruker Ultra Shield 700 instrument, running at $700 \mathrm{MHz}$ for ${ }^{1} \mathrm{H}$ and $176 \mathrm{MHz}$ for ${ }^{13} \mathrm{C}$, respectively. Chemical shifts $(\delta)$ are reported in ppm relative to residual solvent signals $\left(\mathrm{CDCl}_{3}\right.$ : $7.26 \mathrm{ppm}$ for ${ }^{1} \mathrm{H} \mathrm{NMR}, 77.16 \mathrm{ppm}$ for $\left.{ }^{13} \mathrm{C} \mathrm{NMR}\right)$. Mass spectra were recorded on a Bruker Maxis Impact quadrupole-time-of-flight spectrometer using electrospray (ES+) ionization (referenced to the mass of the charged species). Analytical thin layer chromatography (TLC) was performed using pre-coated aluminum-backed plates (Merck Kieselgel 60 F254) and visualized by ultraviolet irradiation or Hanessian's stain. Unless otherwise noted, analytical grade solvents and commercially available reagents were used without further purification. For flash chromatography (FC) silica gel (Silica gel 60, 230-400 mesh, Fluka). The enantiomeric ratio (er) of the products were determined by Ultra Performance Convergence Chromatography ( $\mathrm{UPC}^{2}$ ) using Daicel Chiralpak IA column as chiral stationary phases. Aldehydes 2 were synthesized according to the literature procedure. ${ }^{1}$ Heteroaromatic aldehydes 1 were prepared from the corresponding starting materials following the literature procedure. $^{2}$

(1) Daubresse, N.; Francesch, C.; Rolando, C. Phase transfer Wittig reaction with 1,3-dioxolan2-yl-methyltriphenyl phosphonium salts: An efficient method for vinylogation of aromatic aldehydes. Tetrahedron 1998, 54, 10761-10770.

(2) Bojanowski, J.; Skrzyńska, A.; Albrecht, A. Dearomatizative and Decarboxylative Reaction Cascade in the Aminocatalytic Synthesis of 3,4-Dihydrocoumarins. Asian J. Org. Chem. 2019, 8, 844-848. 


\section{Differentiating catalysis in the $[4+2]$-cycloaddition - general procedure}

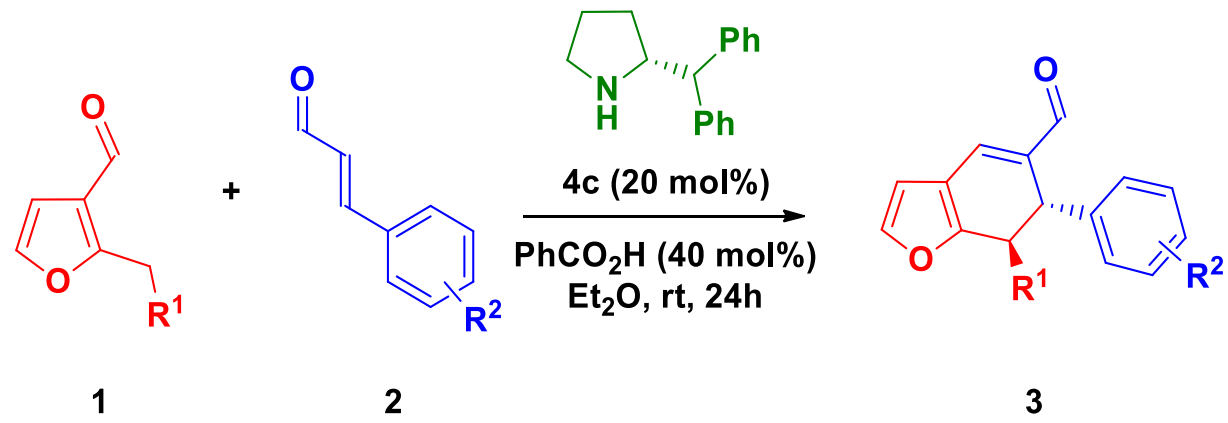

In an ordinary $4 \mathrm{~mL}$ glass vial equipped with a magnetic stirring bar $\alpha, \beta$-unsaturated aldehyde 2 ( $0.12 \mathrm{mmol}, 1.2$ equiv.) and heteroaromatic aldehyde 1 (0.1 mmol, 1 equiv.) were dissolved in $\mathrm{Et}_{2} \mathrm{O}(0.4 \mathrm{~mL})$ and catalyst $4 \mathrm{c}$ (4.7 $\mathrm{mg}, 0.02 \mathrm{mmol}, 0.2$ equiv.) and benzoic acid (4.9 $\mathrm{mg}, 0.04 \mathrm{mmol}, 0.4$ equiv.) were added and the reaction mixture was stirred in room temperature for indicated time. The progress of the reaction was controlled by ${ }^{1} \mathrm{H}$ NMR spectroscopy. After full conversion of the starting material $\mathbf{1}$, the reaction mixture was directly subjected to column chromatography on silica gel (hexanes : diethyl ether 85:15) to afford pure products 3a-o.

\section{(6S,7S)-6,7-Diphenyl-6,7-dihydrobenzofuran-5-carbaldehyde 3a}

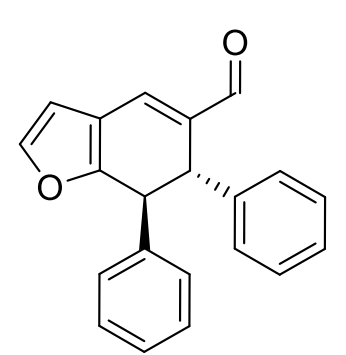

Following the general procedure, using 1a $(18.6 \mathrm{mg})$, product 3a $(>20: 1 \mathrm{dr}$ in a crude reaction mixture) was isolated in $95 \%$ yield $(28.5 \mathrm{mg})$ as lightyellow solid (m.p. $=148-150{ }^{\circ} \mathrm{C}$ after recrystallization from hexane/diethyl ether mixture). ${ }^{1} \mathrm{H}$ NMR $\left(700 \mathrm{MHz}, \mathrm{CDCl}_{3}\right) \delta 9.54(\mathrm{~s}, 1 \mathrm{H})$, $7.46(\mathrm{~s}, 1 \mathrm{H}), 7.42(\mathrm{~d}, J=2.0 \mathrm{~Hz}, 1 \mathrm{H}), 7.31-7.28(\mathrm{~m}, 3 \mathrm{H}), 7.28-7.26(\mathrm{~m}$, 3H), $7.25-7.21(\mathrm{~m}, 2 \mathrm{H}), 7.05-7.04(\mathrm{~m}, 2 \mathrm{H}), 6.60(\mathrm{~d}, J=2.0 \mathrm{~Hz}, 1 \mathrm{H}), 4.44(\mathrm{~s}, 1 \mathrm{H}), 4.34$ (s, 1H). ${ }^{13} \mathrm{C}$ NMR (176 MHz, $\left.\mathrm{CDCl}_{3}\right) \delta 191.3,157.6,144.3,142.6,141.6,139.7,136.5,129.2$ (2C), 129.0 (2C), 127.6, 127.4, 127.0 (2C), 126.8 (2C), 117.6, 108.6, 47.4, 47.1. The er was determined by $\mathrm{UPC}^{2}$ using a chiral Chiralpack IA column gradient from $100 \% \mathrm{CO}_{2}$ up to $40 \%$; $i$ PrOH, flow rate $=2.2 \mathrm{~mL} / \mathrm{min}, 1=295 \mathrm{~nm}$ ) tR = $3.1 \mathrm{~min}$ (major), $2.9 \mathrm{~min}$ (minor), $(>99: 1 \mathrm{er}$ ). $[\alpha]_{\mathrm{D}}^{23}=+625.9\left(\mathrm{c}=1.0, \mathrm{CHCl}_{3}\right)$. HRMS $(\mathrm{ESI}) \mathrm{m} / \mathrm{z}[\mathrm{M}+\mathrm{H}]^{+}$Calcd. for $\mathrm{C}_{21} \mathrm{H}_{17} \mathrm{O}_{2}{ }^{+}: 301.1224$ found: 301.1230 . 


\section{(6S,7S)-6-(4-Nitrophenyl)-7-phenyl-6,7-dihydrobenzofuran-5-carbaldehyde 3b}

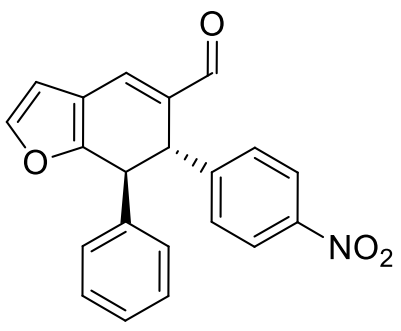

Following the general procedure, using 1a $(18.6 \mathrm{mg})$, product $\mathbf{3 b}$ (10.5:1 $\mathrm{dr}$ in a crude reaction mixture) was isolated in $69 \%$ yield (23.8 mg) as light-yellow oil. ${ }^{1} \mathrm{H}$ NMR $\left(700 \mathrm{MHz}, \mathrm{CDCl}_{3}\right) \delta 9.56(\mathrm{~s}, 1 \mathrm{H})$, $8.15-8.13(\mathrm{~m}, 2 \mathrm{H}), 7.55(\mathrm{~s}, 1 \mathrm{H}), 7.47(\mathrm{~d}, J=2.0 \mathrm{~Hz}, 1 \mathrm{H}), 7.43(\mathrm{~d}, J=$ $8.6 \mathrm{~Hz}, 2 \mathrm{H}), 7.34-7.31(\mathrm{~m}, 2 \mathrm{H}), 7.30-7.27(\mathrm{~m}, 1 \mathrm{H}), 7.06-7.04(\mathrm{~m}$, 2H), $6.65(\mathrm{~d}, J=2.0 \mathrm{~Hz}, 1 \mathrm{H}), 4.53(\mathrm{~s}, 1 \mathrm{H}), 4.31(\mathrm{~s}, 1 \mathrm{H}) .{ }^{13} \mathrm{C} \mathrm{NMR}\left(176 \mathrm{MHz}, \mathrm{CDCl}_{3}\right) \delta 190.9$, 157.0, 149.7, 147.4, 144.9, 140.7, 140.4, 135.3, 129.3 (2C), 128.0 (2C), 127.9, 126.7 (2C), 124.3 (2C), 117.7, 108.7, 47.0, 46.9. The er was determined by $\mathrm{UPC}^{2}$ using a chiral Chiralpack IA column gradient from $100 \% \mathrm{CO}_{2}$ up to $40 \%$; $i-\mathrm{PrOH}$, flow rate $\left.=2.2 \mathrm{~mL} / \mathrm{min}, 1=358 \mathrm{~nm}\right) \mathrm{tR}=$ $4.0 \mathrm{~min}$ (major), $3.7 \mathrm{~min}$ (minor), $(97: 3 \mathrm{er}) .[\alpha]_{\mathrm{D}}^{22}=+776.8\left(\mathrm{c}=1.0, \mathrm{CHCl}_{3}\right) . \mathrm{HRMS}(\mathrm{ESI}) \mathrm{m} / \mathrm{z}$ $[\mathrm{M}+\mathrm{H}]^{+}$Calcd. for $\mathrm{C}_{21} \mathrm{H}_{16} \mathrm{NO}_{4}{ }^{+}: 346.1074$; found: 346.1085 .

\section{(6S,7S)-6-(4-Chlorophenyl)-7-phenyl-6,7-dihydrobenzofuran-5-carbaldehyde 3c}

Following the general procedure, using 1a $(18.6 \mathrm{mg})$, product 3c<smiles>O=CC1=Cc2ccoc2C(c2ccccc2)[C@H]1c1ccc(Cl)cc1</smiles>
(>20:1 dr in a crude reaction mixture) was isolated in $71 \%$ yield (23.7 $\mathrm{mg})$ as light-yellow oil. ${ }^{1} \mathrm{H} \mathrm{NMR}\left(700 \mathrm{MHz}, \mathrm{CDCl}_{3}\right) \delta 9.52(\mathrm{~s}, 1 \mathrm{H})$, $7.46(\mathrm{~s}, 1 \mathrm{H}), 7.42$ (d, $J=2.0 \mathrm{~Hz}, 1 \mathrm{H}), 7.30-7.27(\mathrm{~m}, 2 \mathrm{H}), 7.25-7.21$ $(\mathrm{m}, 3 \mathrm{H}), 7.19-7.17(\mathrm{~m}, 2 \mathrm{H}), 7.04-7.01(\mathrm{~m}, 2 \mathrm{H}), 6.60(\mathrm{~d}, J=2.0 \mathrm{~Hz}$, $1 \mathrm{H}), 4.39$ (s, 1H), 4.27 (s, 1H). ${ }^{13} \mathrm{C} \mathrm{NMR}\left(176 \mathrm{MHz}, \mathrm{CDCl}_{3}\right) \delta$ 191.1, 157.3, 144.5, 141.2, 141.0, 139.8, 136.1, 133.2, 129.2 (2C), 129.1 (2C), 128.4 (2C), 127.7, 126.7 (2C), 117.6, 108.6, 47.3, 46.4. The er was determined by $\mathrm{UPC}^{2}$ using a chiral Chiralpack IA column gradient from $100 \% \mathrm{CO}_{2}$ up to $40 \%$; $i-\mathrm{PrOH}$, flow rate $\left.=2.2 \mathrm{~mL} / \mathrm{min}, 1=330 \mathrm{~nm}\right) \mathrm{tR}=$ $3.6 \mathrm{~min}$ (major), $3.2 \mathrm{~min}$ (minor), (98:2 er). $[\alpha]_{\mathrm{D}}^{23}=+753.3\left(\mathrm{c}=1.0, \mathrm{CHCl}_{3}\right) . \mathrm{HRMS}(\mathrm{ESI}) \mathrm{m} / \mathrm{z}$ $[\mathrm{M}+\mathrm{H}]^{+}$Calcd. for $\mathrm{C}_{21} \mathrm{H}_{16} \mathrm{ClO}_{2}{ }^{+}$: 335.0761 ; found: 335.0769 .

\section{(6S,7S)-7-Phenyl-6-(p-tolyl)-6,7-dihydrobenzofuran-5-carbaldehyde 3d}

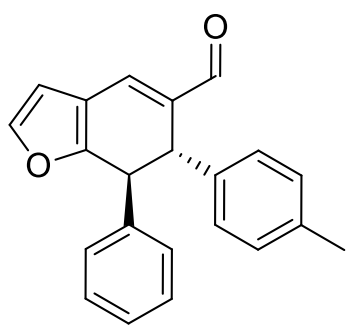

Following the general procedure, using 1a (18.6 mg), product 3d (>20:1 $\mathrm{dr}$ in a crude reaction mixture) was isolated in $69 \%$ yield $(21.7 \mathrm{mg})$ as light-yellow oil. ${ }^{1} \mathrm{H}$ NMR $\left(700 \mathrm{MHz}, \mathrm{CDCl}_{3}\right) \delta 9.53$ (s, 1H), $7.43(\mathrm{~s}, 1 \mathrm{H})$, $7.41(\mathrm{~d}, J=2.0 \mathrm{~Hz}, 1 \mathrm{H}), 7.28-7.27(\mathrm{~m}, 2 \mathrm{H}), 7.25-7.21(\mathrm{~m}, 1 \mathrm{H}), 7.16-$ 
$7.13(\mathrm{~m}, 2 \mathrm{H}), 7.07-7.06(\mathrm{~m}, 2 \mathrm{H}), 7.05-7.04(\mathrm{~m}, 2 \mathrm{H}), 6.59(\mathrm{~d}, J=2.0 \mathrm{~Hz}, 1 \mathrm{H}), 4.40(\mathrm{~d}, J=1.4$ $\mathrm{Hz}, 1 \mathrm{H}), 4.32$ (s, 1H), 2.30 (s, 3H). ${ }^{13} \mathrm{C}$ NMR $\left(176 \mathrm{MHz}, \mathrm{CDCl}_{3}\right) \delta 191.3,157.6,144.3$, 141.6, 139.7, 139.5, 137.0, 136.6, 129.6 (2C), 129.1 (2C), 127.5, 126.9 (2C), 126.8 (2C), 117.6, 108.6, $47.5,46.7,21.2$. The er was determined by UPC $^{2}$ using a chiral Chiralpack IA column gradient from $100 \% \mathrm{CO}_{2}$ up to $40 \%$; $i-\mathrm{PrOH}$, flow rate $=2.2 \mathrm{~mL} / \mathrm{min}, 1=230 \mathrm{~nm}$ ) tR $=3.3 \mathrm{~min}$ (major), $3.0 \mathrm{~min}$ (minor), (98:2 er). $[\alpha]_{\mathrm{D}}^{23}=+329.3\left(\mathrm{c}=1.0, \mathrm{CHCl}_{3}\right)$. HRMS (ESI) $\mathrm{m} / \mathrm{z}[\mathrm{M}+\mathrm{H}]^{+} \mathrm{Calcd}$. for $\mathrm{C}_{22} \mathrm{H}_{19} \mathrm{O}_{2}^{+}$: 315.1380; found: 315.1388 .

\section{(6S,7S)-6-(4-Methoxyphenyl)-7-phenyl-6,7-dihydrobenzofuran-5-carbaldehyde 3e}

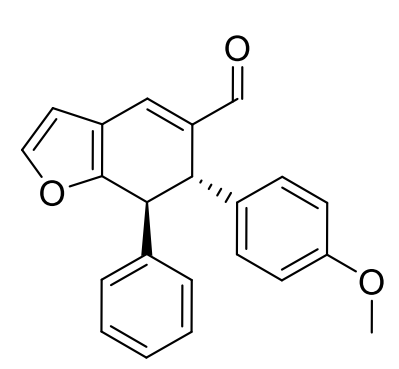

Following the general procedure, using 1a (18.6 mg), product 3e (>20:1

$\mathrm{dr}$ in a crude reaction mixture) was isolated in $76 \%$ yield $(25.1 \mathrm{mg})$ as light-yellow oil. ${ }^{1} \mathrm{H}$ NMR $\left(700 \mathrm{MHz}, \mathrm{CDCl}_{3}\right) \delta 9.52(\mathrm{~s}, 1 \mathrm{H}), 7.41-7.40$ $(\mathrm{m}, 2 \mathrm{H}), 7.28-7.27(\mathrm{~m}, 2 \mathrm{H}), 7.24-7.21(\mathrm{~m}, 1 \mathrm{H}), 7.18-7.16(\mathrm{~m}, 2 \mathrm{H})$, $7.05-7.01(\mathrm{~m}, 2 \mathrm{H}), 6.79-6.77(\mathrm{~m}, 2 \mathrm{H}), 6.59(\mathrm{~d}, J=2.1 \mathrm{~Hz}, 1 \mathrm{H}), 4.37$ (s, $1 \mathrm{H}), 4.30(\mathrm{~s}, 1 \mathrm{H}), 3.76(\mathrm{~s}, 3 \mathrm{H}) .{ }^{13} \mathrm{C} \mathrm{NMR}\left(176 \mathrm{MHz}, \mathrm{CDCl}_{3}\right) \delta 191.4$, 158.9, 157.6, 144.3 141.6, 139.3, 136.8, 134.8, 129.1 (2C), 128.1 (2C), 127.5, 126.8 (2C), 117.6, 114.3 (2C), 108.6, 55.4, 47.5, 46.3. The er was determined by UPC ${ }^{2}$ using a chiral Chiralpack IA column gradient from $100 \% \mathrm{CO}_{2}$ up to $40 \%$; $i-\mathrm{PrOH}$, flow rate $=2.2 \mathrm{~mL} / \mathrm{min}, 1=215 \mathrm{~nm}$ ) $\mathrm{tR}=$ $3.6 \mathrm{~min}$ (major), $3.3 \mathrm{~min}$ (minor), (98:2 er). $[\alpha]_{\mathrm{D}}{ }^{23}=+593.4\left(\mathrm{c}=1.0, \mathrm{CHCl}_{3}\right)$. HRMS (ESI) $\mathrm{m} / \mathrm{z}$ $[\mathrm{M}+\mathrm{H}]^{+}$Calcd. for $\mathrm{C}_{22} \mathrm{H}_{19} \mathrm{O}_{3}{ }^{+}$: 331.1329 ; found: 331.1337 .

\section{(6S,7S)-6-(3-Methoxyphenyl)-7-phenyl-6,7-dihydrobenzofuran-5-carbaldehyde $3 f$}

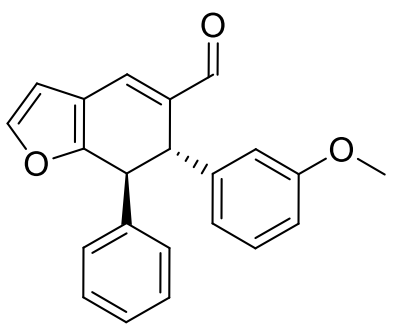

Following the general procedure, using 1a $(18.6 \mathrm{mg})$, product $\mathbf{3 f}(>20: 1$ $\mathrm{dr}$ in a crude reaction mixture) was isolated in $68 \%$ yield $(22.4 \mathrm{mg})$ as light-yellow oil. ${ }^{1} \mathrm{H}$ NMR (700 MHz, $\left.\mathrm{CDCl}_{3}\right) \delta 9.53$ (s, 1H), 7.45 (s, $1 \mathrm{H}), 7.40(\mathrm{~d}, J=2.0 \mathrm{~Hz}, 1 \mathrm{H}), 7.30-7.26(\mathrm{~m}, 2 \mathrm{H}), 7.25-7.22(\mathrm{~m}, 1 \mathrm{H})$, $7.19-7.17(\mathrm{~m}, 1 \mathrm{H}), 7.06-7.03(\mathrm{~m}, 2 \mathrm{H}), 6.88-6.86(\mathrm{~m}, 1 \mathrm{H}), 6.81(\mathrm{~d}$, $J=2.0 \mathrm{~Hz}, 1 \mathrm{H}), 6.78-6.76(\mathrm{~m}, 1 \mathrm{H}), 6.58(\mathrm{~d}, J=2.2 \mathrm{~Hz}, 1 \mathrm{H}), 4.41(\mathrm{~s}, 1 \mathrm{H}), 4.35(\mathrm{~s}, 1 \mathrm{H}), 3.76(\mathrm{~s}$, 3H). ${ }^{13} \mathrm{C}$ NMR $\left(176 \mathrm{MHz}, \mathrm{CDCl}_{3}\right) \delta 191.3,160.0,157.6,144.3,144.1,141.6,139.8,136.3$, 130.0, 129.2 (2C), 127.5, 126.8 (2C), 119.4, 117.5, 113.2, 112.3, 108.6, 55.3, 47.3, 47.0. The er was determined by $\mathrm{UPC}^{2}$ using a chiral Chiralpack IA column gradient from $100 \% \mathrm{CO}_{2}$ up to 
$40 \% ; i-\mathrm{PrOH}$, flow rate $=2.2 \mathrm{~mL} / \mathrm{min}, 1=251 \mathrm{~nm}$ ) tR $=3.2 \mathrm{~min}$ (major), $3.1 \mathrm{~min}$ (minor), $(98: 2$ er). $[\alpha]_{D}^{23}=+468.0\left(\mathrm{c}=1.0, \mathrm{CHCl}_{3}\right)$. HRMS (ESI) $\mathrm{m} / \mathrm{z}[\mathrm{M}+\mathrm{H}]^{+}$Calcd. for $\mathrm{C}_{22} \mathrm{H}_{19} \mathrm{O}_{3}{ }^{+}$: 331.1329; found: 331.1338 .

\section{(6S,7S)-6-(2-methoxyphenyl)-7-phenyl-6,7-dihydrobenzofuran-5-carbaldehyde 3g}

Following the general procedure, using $1 \mathbf{a}(18.6 \mathrm{mg})$, product $\mathbf{3 g}(>20: 1 \mathrm{dr}$<smiles>COc1ccccc1C1C(C=O)=Cc2ccoc2C1c1ccccc1</smiles>
in a crude reaction mixture) was isolated in $57 \%$ yield $(18.8 \mathrm{mg})$ as lightyellow oil. ${ }^{1} \mathrm{H}$ NMR $\left(700 \mathrm{MHz}, \mathrm{CDCl}_{3}\right) \delta 9.54(\mathrm{~s}, 1 \mathrm{H}), 7.60$ (s, $\left.1 \mathrm{H}\right), 7.36-$ $7.35(\mathrm{~d}, J=1.8 \mathrm{~Hz}, 1 \mathrm{H}), 7.29-7.26(\mathrm{~m}, 2 \mathrm{H}), 7.23-7.19(\mathrm{~m}, 1 \mathrm{H}), 7.20-$ $7.19(\mathrm{~m}, 1 \mathrm{H}), 7.10-7.06(\mathrm{~m}, 2 \mathrm{H}), 6.95-6.94(\mathrm{~m}, 1 \mathrm{H}), 6.88(\mathrm{~d}, J=1.8 \mathrm{~Hz}$, 1H), $6.78-6.74(\mathrm{~m}, 1 \mathrm{H}), 6.59(\mathrm{~s}, 1 \mathrm{H}), 4.86(\mathrm{~s}, 1 \mathrm{H}), 4.28(\mathrm{~s}, 1 \mathrm{H}), 4.00(\mathrm{~s}$, 3H). ${ }^{13} \mathrm{C}$ NMR (176 MHz, $\left.\mathrm{CDCl}_{3}\right) \delta 191.3,158.2,157.0,144.1,141.6,141.2,135.4,128.8$ (2C), $128.4,128.3,127.2,127.0$ (2C), 126.9, 120.4, 117.7, 111.2, 108.5, 55.6, 45.9, 40.7. The er was determined by UPC ${ }^{2}$ using a chiral Chiralpack IA column gradient from $100 \% \mathrm{CO}_{2}$ up to $40 \%$; $i$ PrOH, flow rate $=2.2 \mathrm{~mL} / \mathrm{min}, 1=339 \mathrm{~nm}$ ) $\mathrm{tR}=3.2 \mathrm{~min}$ (major), $3.0 \mathrm{~min}$ (minor), $(>99: 1 \mathrm{er}$ ). $[\alpha]_{\mathrm{D}}^{23}=+402.2\left(\mathrm{c}=1.0, \mathrm{CHCl}_{3}\right)$. HRMS (ESI) $\mathrm{m} / \mathrm{z}[\mathrm{M}+\mathrm{H}]^{+}$Calcd. for $\mathrm{C}_{22} \mathrm{H}_{19} \mathrm{O}_{3}{ }^{+}: 331.1329$ found: 331.1340 .

(6R,7S)-6-(2,4-Dichlorophenyl)-7-phenyl-6,7-dihydrobenzofuran-5-carbaldehyde 3h

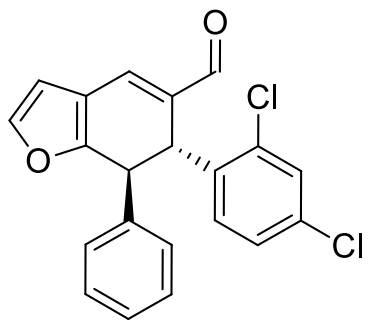

Following the general procedure, using 1a $(18.6 \mathrm{mg})$, product $\mathbf{3 h}(>20: 1$ $\mathrm{dr}$ in a crude reaction mixture) was isolated in $70 \%$ yield $(25.8 \mathrm{mg})$ as light-yellow oil. ${ }^{1} \mathrm{H}$ NMR $\left(700 \mathrm{MHz}, \mathrm{CDCl}_{3}\right) \delta 9.57$ (s, 1H), 7.43 (s, $1 \mathrm{H}), 7.40(\mathrm{~d}, J=2.0 \mathrm{~Hz}, 1 \mathrm{H}), 7.34-7.31(\mathrm{~m}, 1 \mathrm{H}), 7.28-7.26(\mathrm{~m}, 2 \mathrm{H})$, $7.25-7.20(\mathrm{~m}, 1 \mathrm{H}), 7.05-7.01(\mathrm{~m}, 2 \mathrm{H}), 6.56(\mathrm{~d}, J=1.9 \mathrm{~Hz}, 1 \mathrm{H}), 6.22$ $(\mathrm{dd}, J=3.2,1.9 \mathrm{~Hz}, 1 \mathrm{H}), 5.96(\mathrm{~d}, J=3.2 \mathrm{~Hz}, 1 \mathrm{H}), 4.61(\mathrm{~s}, 1 \mathrm{H}), 4.60(\mathrm{~s}, 1 \mathrm{H}) .{ }^{13} \mathrm{C}$ NMR $(176$ $\left.\mathrm{MHz}, \mathrm{CDCl}_{3}\right) \delta 190.8,157.8,154.1,144.2$ (2C), 142.0 (2C), 140.5, 140.0, 133.5, 129.1, 127.6 (2C), 126.9 (2C), 117.2, 110.3, 108.7, 105.8, 43.9, 40.5. The er was determined by $\mathrm{UPC}^{2}$ using a chiral Chiralpack IA column gradient from $100 \% \mathrm{CO}_{2}$ up to $40 \%$; $\mathrm{ACN}$, flow rate $=2.2 \mathrm{~mL} / \mathrm{min}$, $1=235 \mathrm{~nm}) \mathrm{tR}=3.0 \mathrm{~min}$ (major), $3.4 \mathrm{~min}$ (minor), (90:10 er). $[\alpha]_{\mathrm{D}}{ }^{23}=+591.1\left(\mathrm{c}=1.0, \mathrm{CHCl}_{3}\right)$. HRMS (ESI) $\mathrm{m} / \mathrm{z}[\mathrm{M}+\mathrm{H}]^{+}$Calcd. for $\mathrm{C}_{21} \mathrm{H}_{15} \mathrm{Cl}_{2} \mathrm{O}_{2}{ }^{+}$: + 369.0444; found: 369.0455 . 


\section{(6R,7S)-6-(Furan-2-yl)-7-phenyl-6,7-dihydrobenzofuran-5-carbaldehyde 3i}

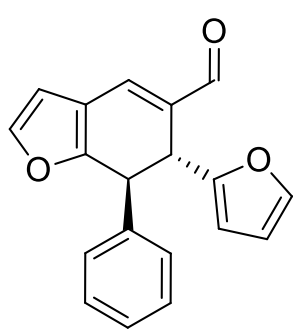

Following the general procedure, using 1a $(18.6 \mathrm{mg})$, product 3i (>20:1 dr in a crude reaction mixture) was isolated in $64 \%$ yield $(18.6 \mathrm{mg})$ as light-yellow oil. ${ }^{1} \mathrm{H}$ NMR $\left(700 \mathrm{MHz}, \mathrm{CDCl}_{3}\right) \delta 9.55$ (s, 1H), 7.64 (s, 1H), 7.48 (d, J=2.0 $\mathrm{Hz}, 1 \mathrm{H}), 7.39$ (d, $J=2.1 \mathrm{~Hz}, 1 \mathrm{H}), 7.29-7.27$ (m, 2H), $7.25-7.22(\mathrm{~m}, 1 \mathrm{H})$, $7.14-7.12(\mathrm{~m}, 2 \mathrm{H}), 7.05(\mathrm{dd}, J=8.4,2.0 \mathrm{~Hz}, 1 \mathrm{H}), 6.91(\mathrm{~d}, J=8.4 \mathrm{~Hz}, 1 \mathrm{H})$, $6.60(\mathrm{~d}, J=2.1 \mathrm{~Hz}, 1 \mathrm{H}), 4.88(\mathrm{~s}, 1 \mathrm{H}), 4.20(\mathrm{~s}, 1 \mathrm{H}) .{ }^{13} \mathrm{C} \mathrm{NMR}\left(176 \mathrm{MHz}, \mathrm{CDCl}_{3}\right) \delta 190.7,157.4$, 144.6, 141.2, 140.4, 136.5, 135.3, 134.5, 133.7, 130.3, 129.2, 128.8, 127.7, 127.3, 127.2, 117.6, $108.5,46.0,42.8$. The er was determined by $\mathrm{UPC}^{2}$ using a chiral Chiralpack IA column gradient from $100 \% \mathrm{CO}_{2}$ up to $40 \%$; $\mathrm{ACN}$, flow rate $=2.2 \mathrm{~mL} / \mathrm{min}, 1=237 \mathrm{~nm}$ ) $\mathrm{tR}=2.5 \mathrm{~min}$ (major), 2.7 $\min$ (minor), $(95: 5 \mathrm{er}) .[\alpha]_{\mathrm{D}}^{23}=+614.1\left(\mathrm{c}=1.0, \mathrm{CHCl}_{3}\right)$. HRMS (ESI) m/z $[\mathrm{M}+\mathrm{H}]^{+}$Calcd. for $\mathrm{C}_{19} \mathrm{H}_{15} \mathrm{O}_{3}{ }^{+}$: 291.1016; found: 291.1022 .

\section{(6S,7S)-7-(4-Fluorophenyl)-6-phenyl-6,7-dihydrobenzofuran-5-carbaldehyde 3j}

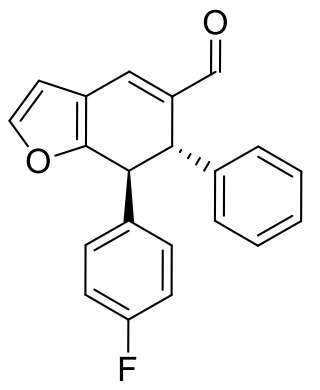

Following the general procedure, using $\mathbf{1 b}(20.4 \mathrm{mg})$, product $\mathbf{3 j}$ (>20:1 dr in a crude reaction mixture) was isolated in $90 \%$ yield $(28.6 \mathrm{mg})$ as lightyellow oil. ${ }^{1} \mathrm{H}$ NMR $\left(700 \mathrm{MHz}, \mathrm{CDCl}_{3}\right) \delta 9.56(\mathrm{~s}, 1 \mathrm{H}), 7.48(\mathrm{~s}, 1 \mathrm{H}), 7.45(\mathrm{~d}$, $J=2.1 \mathrm{~Hz}, 1 \mathrm{H}), 7.31-7.22(\mathrm{~m}, 5 \mathrm{H}), 7.06-6.97(\mathrm{~m}, 4 \mathrm{H}), 6.63(\mathrm{~d}, J=2.1$ $\mathrm{Hz}, 1 \mathrm{H}), 4.41(\mathrm{~s}, 1 \mathrm{H}), 4.35(\mathrm{~s}, 1 \mathrm{H}) .{ }^{13} \mathrm{C} \mathrm{NMR}\left(176 \mathrm{MHz}, \mathrm{CDCl}_{3}\right) \delta 191.3$, $162.8(\mathrm{~d}, J=246.1 \mathrm{~Hz}, 2 \mathrm{C}), 161.5,157.3,144.5,142.3,139.6,137.3,136.3$, 129.0, 128.4 (d, $J=8.2 \mathrm{~Hz}, 2 \mathrm{C}), 127.5,127.0,117.6,116.0$ (d, $J=21.2 \mathrm{~Hz}, 2 \mathrm{C}), 108.6,47.2$, 46.5. The er was determined by $\mathrm{UPC}^{2}$ using a chiral Chiralpack IA column gradient from $100 \%$ $\mathrm{CO}_{2}$ up to $40 \%$; $i$-PrOH, flow rate $=2.2 \mathrm{~mL} / \mathrm{min}, 1=244 \mathrm{~nm}$ ) $\mathrm{tR}=3.0 \mathrm{~min}$ (major), $2.7 \mathrm{~min}$ (minor), (97:3 er). $[\alpha]_{\mathrm{D}}^{23}=+734.4\left(\mathrm{c}=1.0, \mathrm{CHCl}_{3}\right)$. HRMS (ESI) $\mathrm{m} / \mathrm{z}[\mathrm{M}+\mathrm{H}]^{+}$Calcd. for $\mathrm{C}_{21} \mathrm{H}_{16} \mathrm{FO}_{2}^{+}$: 319.1129; found: 319.1141 .

\section{(6S,7S)-6-Phenyl-7-(p-tolyl)-6,7-dihydrobenzofuran-5-carbaldehyde 3k}

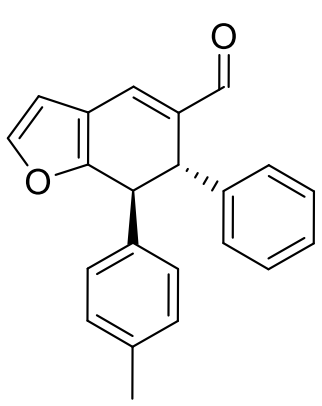

Following the general procedure, using 1c $(20.0 \mathrm{mg})$, product $3 \mathbf{k}(15: 1 \mathrm{dr}$ in a crude reaction mixture) was isolated in $56 \%$ yield $(17.6 \mathrm{mg})$ as lightyellow oil. ${ }^{1} \mathrm{H}$ NMR $\left(700 \mathrm{MHz}, \mathrm{CDCl}_{3}\right) \delta 9.54(\mathrm{~s}, 1 \mathrm{H}), 7.45(\mathrm{~s}, 1 \mathrm{H}), 7.41$ $(\mathrm{dd}, J=2.0,0.6 \mathrm{~Hz}, 1 \mathrm{H}), 7.27(\mathrm{~s}, 4 \mathrm{H}), 7.25-7.21(\mathrm{~m}, 1 \mathrm{H}), 7.11-7.08(\mathrm{~m}$, 
2H), $6.96-6.93(\mathrm{~m}, 2 \mathrm{H}), 6.59(\mathrm{dd}, J=2.0,0.6 \mathrm{~Hz}, 1 \mathrm{H}), 4.43(\mathrm{~d}, J=1.4 \mathrm{~Hz}, 1 \mathrm{H}), 4.33-4.30(\mathrm{~s}$, 1H), 2.32 (s, 3H). ${ }^{13} \mathrm{C}$ NMR $\left(176 \mathrm{MHz}, \mathrm{CDCl}_{3}\right) \delta 191.3,157.9,144.3,142.6,139.8,138.6$, 137.2, 136.4, 129.8 (2C), 129.0 (2C), 127.3, 127.0 (2C), 126.6 (2C), 117.5, 108.5, 47.1, 47.0, 21.2. The er was determined by $\mathrm{UPC}^{2}$ using a chiral Chiralpack IA column gradient from $100 \%$ $\mathrm{CO}_{2}$ up to $40 \% ; i-\mathrm{PrOH}$, flow rate $=2.2 \mathrm{~mL} / \mathrm{min}, 1=230 \mathrm{~nm}$ ) $\mathrm{tR}=3.1 \mathrm{~min}$ (major), $2.9 \mathrm{~min}$ (minor), (96:4 er). $[\alpha]_{\mathrm{D}}{ }^{23}=+849.4\left(\mathrm{c}=1.0, \mathrm{CHCl}_{3}\right)$. HRMS $(\mathrm{ESI}) \mathrm{m} / \mathrm{z}[\mathrm{M}+\mathrm{H}]^{+}$Calcd. for $\mathrm{C}_{22} \mathrm{H}_{19} \mathrm{O}_{2}{ }^{+}$: 315.1380; found: 315.1387 .

\section{(6S,7S)-7-(3-Methoxyphenyl)-6-phenyl-6,7-dihydrobenzofuran-5-carbaldehyde 31}

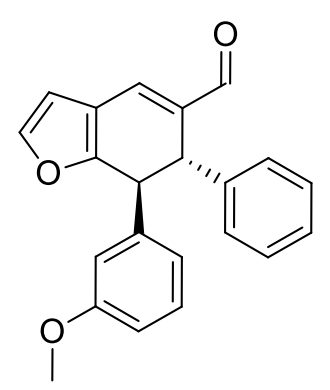

Following the general procedure, using $\mathbf{1 d}(21.6 \mathrm{mg})$, product $\mathbf{3 l}(>20: 1 \mathrm{dr}$ in a crude reaction mixture) was isolated in $93 \%$ yield $(30.7 \mathrm{mg})$ as lightyellow oil. ${ }^{1} \mathrm{H}$ NMR $\left(700 \mathrm{MHz}, \mathrm{CDCl}_{3}\right) \delta 9.54(\mathrm{~s}, 1 \mathrm{H}), 7.45(\mathrm{~s}, 1 \mathrm{H}), 7.42(\mathrm{~d}$, $J=2.1 \mathrm{~Hz}, 1 \mathrm{H}), 7.29-7.26(\mathrm{~m}, 4 \mathrm{H}), 7.24-7.18(\mathrm{~m}, 2 \mathrm{H}), 6.79-6.77(\mathrm{~m}$, $1 \mathrm{H}), 6.68-6.67(\mathrm{~m}, 1 \mathrm{H}), 6.59(\mathrm{~d}, J=2.1 \mathrm{~Hz}, 1 \mathrm{H}), 6.58-6.57(\mathrm{~m}, 1 \mathrm{H}), 4.44$ (s, 1H), $4.32(\mathrm{~s}, 1 \mathrm{H}), 3.77$ (s, 3H). ${ }^{13} \mathrm{C}$ NMR $\left(176 \mathrm{MHz}, \mathrm{CDCl}_{3}\right) \delta 191.3$,

160.1, 157.4, 144.4, 143.0, 142.5, 139.7, 136.5, 130.2, 129.0 (2C), 127.4, 127.0 (2C), 119.1, 117.6, 113.0, 112.3, 108.6, 55.3, 47.3, 47.0. The er was determined by $\mathrm{UPC}^{2}$ using a chiral Chiralpack IA column gradient from $100 \% \mathrm{CO}_{2}$ up to $40 \% ; i-\mathrm{PrOH}$, flow rate $=2.2 \mathrm{~mL} / \mathrm{min}, 1=$ $227 \mathrm{~nm}) \mathrm{tR}=3.2 \mathrm{~min}$ (major), $3.1 \mathrm{~min}$ (minor), (81:19 er). $[\alpha]_{\mathrm{D}}^{23}=+692.2\left(\mathrm{c}=1.0, \mathrm{CHCl}_{3}\right)$. HRMS (ESI) $\mathrm{m} / \mathrm{z}[\mathrm{M}+\mathrm{H}]^{+}$Calcd. for $\mathrm{C}_{22} \mathrm{H}_{19} \mathrm{O}_{3}{ }^{+}$: 331.1329; found: 331.1343 .

\section{(6S,7S)-6-Phenyl-7-(0-tolyl)-6,7-dihydrobenzofuran-5-carbaldehyde 3m}

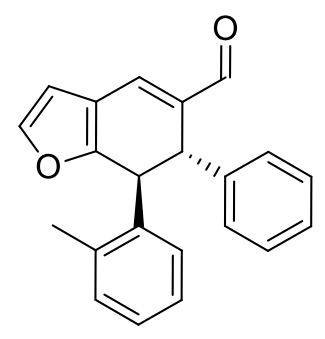

Following the general procedure, using 1 e $(20.0 \mathrm{mg})$, product $3 \mathbf{m}(6: 1 \mathrm{dr}$ in a crude reaction mixture) was isolated in $67 \%$ yield $(21.0 \mathrm{mg})$ as lightyellow oil. ${ }^{1} \mathrm{H}$ NMR $\left(700 \mathrm{MHz}, \mathrm{CDCl}_{3}\right) \delta 9.51(\mathrm{~s}, 1 \mathrm{H}), 7.44(\mathrm{~d}, J=3.6 \mathrm{~Hz}$, $2 \mathrm{H}), 7.32-7.30(\mathrm{~m}, 2 \mathrm{H}), 7.29-7.26(\mathrm{~m}, 2 \mathrm{H}), 7.26-7.23(\mathrm{~m}, 2 \mathrm{H}), 7.14(\mathrm{td}$, $J=7.6,1.3 \mathrm{~Hz}, 1 \mathrm{H}), 7.05(\mathrm{td}, J=7.6,1.3 \mathrm{~Hz}, 1 \mathrm{H}), 6.63(\mathrm{~d}, J=2.0 \mathrm{~Hz}, 1 \mathrm{H})$, $6.60(\mathrm{dd}, J=7.6,1.3 \mathrm{~Hz}, 1 \mathrm{H}), 4.59$ (d, $J=1.3 \mathrm{~Hz}, 1 \mathrm{H}), 4.28$ (d, $J=1.3 \mathrm{~Hz}, 1 \mathrm{H}), 2.51$ (s, 3H). ${ }^{13} \mathrm{C}$ NMR $\left(176 \mathrm{MHz}, \mathrm{CDCl}_{3}\right) \delta 191.3,157.9,146.9,144.3,142.9,139.5,139.3,136.7,134.9$, 131.3, 129.0, 127.4, 127.3, 127.0, 126.6, 126.5, 118.4, 108.5, 85.3, 46.4, 43.1, 27.6. The er was determined by $\mathrm{UPC}^{2}$ using a chiral Chiralpack IA column gradient from $100 \% \mathrm{CO}_{2}$ up to $40 \%$; $i$ - 
$\mathrm{PrOH}$, flow rate $=2.2 \mathrm{~mL} / \mathrm{min}, 1=248 \mathrm{~nm}$ ) $\mathrm{tR}=2.7 \mathrm{~min}$ (major), $2.9 \mathrm{~min}$ (minor), $(>99: 1 \mathrm{er}$ ). $[\alpha]_{\mathrm{D}}^{23}=+496.5\left(\mathrm{c}=1.0, \mathrm{CHCl}_{3}\right)$. HRMS (ESI) $\mathrm{m} / \mathrm{z}[\mathrm{M}+\mathrm{H}]^{+}$Calcd. for $\mathrm{C}_{22} \mathrm{H}_{19} \mathrm{O}_{2}{ }^{+}: 315.1380$; found: 315.1388 .

(6S,7R)-6-Phenyl-7-vinyl-6,7-dihydrobenzofuran-5-carbaldehyde 3n<smiles>C=C[C@H]1c2occc2C=C(C=O)[C@H]1c1ccccc1</smiles>

Following the general procedure, using 1 f $(13.6 \mathrm{mg})$, product 3n $(>20: 1 \mathrm{dr}$ in a crude reaction mixture) was isolated in $91 \%$ yield $(22.8 \mathrm{mg})$ as lightyellow oil. ${ }^{1} \mathrm{H} \mathrm{NMR}\left(700 \mathrm{MHz}, \mathrm{CDCl}_{3}\right) \delta 9.55$ (s, 1H), $7.42(\mathrm{~d}, J=2.0 \mathrm{~Hz}$, $1 \mathrm{H}), 7.36(\mathrm{~s}, 1 \mathrm{H}), 7.23-7.19(\mathrm{~m}, 2 \mathrm{H}), 7.19-7.16(\mathrm{~m}, 3 \mathrm{H}), 6.53(\mathrm{dd}, J=$ 2.0, $0.6 \mathrm{~Hz}, 1 \mathrm{H}), 5.85$ (ddd, $J=17.0,10.1,6.7 \mathrm{~Hz}, 1 \mathrm{H}), 5.05(\mathrm{dt}, J=10.1$, $1.1 \mathrm{~Hz}, 1 \mathrm{H}), 4.88(\mathrm{ddd}, J=17.0,1.4,1.1 \mathrm{~Hz}, 1 \mathrm{H}), 4.28(\mathrm{~d}, J=1.1 \mathrm{~Hz}, 1 \mathrm{H}), 3.76(\mathrm{dd}, J=6.8,1.4$ $\mathrm{Hz}, 1 \mathrm{H}) .{ }^{13} \mathrm{C} \mathrm{NMR}\left(176 \mathrm{MHz}, \mathrm{CDCl}_{3}\right) \delta 191.5,157.2,144.1,141.5,139.9,136.9,136.2,128.8$ (2C), 127.3, 127.1 (2C), 117.0, 115.7, 108.6, 45.4, 43.7. The er was determined by $\mathrm{UPC}^{2}$ using a chiral Chiralpack IA column gradient from $100 \% \mathrm{CO}_{2}$ up to $40 \%$; $\mathrm{ACN}$, flow rate $=2.2 \mathrm{~mL} / \mathrm{min}$, $1=228 \mathrm{~nm}$ ) $\mathrm{tR}=2.5 \mathrm{~min}$ (major), $2.1 \mathrm{~min}$ (minor), $(94: 6 \mathrm{er}) .[\alpha]_{\mathrm{D}}{ }^{23}=+85.6\left(\mathrm{c}=1.0, \mathrm{CHCl}_{3}\right)$. HRMS (ESI) m/z [M+H] $]^{+}$Calcd. for $\mathrm{C}_{17} \mathrm{H}_{15} \mathrm{O}_{2}{ }^{+}:$251.1067; found: 251.1074.

\section{(3S,4S)-3,4-Diphenyl-3,4-dihydrodibenzo[b,d]furan-2-carbaldehyde 30}

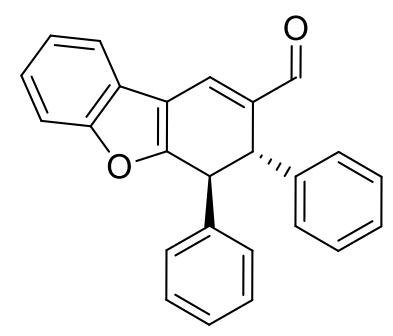

Following the general procedure, using 19 (23.6 mg), product 3o (>20:1 $\mathrm{dr}$ in a crude reaction mixture) was isolated in $86 \%$ yield $(30.1 \mathrm{mg})$ as light-yellow oil. ${ }^{1} \mathrm{H}$ NMR $\left(700 \mathrm{MHz}, \mathrm{CDCl}_{3}\right){ }^{1} \mathrm{H} \mathrm{NMR}(700 \mathrm{MHz}$, $\left.\mathrm{CDCl}_{3}\right) \delta 9.66(\mathrm{~s}, 1 \mathrm{H}), 7.79-7.76(\mathrm{~m}, 2 \mathrm{H}), 7.47(\mathrm{dt}, J=8.1,0.9 \mathrm{~Hz}$, $1 \mathrm{H}), 7.39(\mathrm{td}, J=7.5,1.1 \mathrm{~Hz}, 1 \mathrm{H}), 7.36-7.34(\mathrm{~m}, 1 \mathrm{H}), 7.32-7.28(\mathrm{~m}$, $4 \mathrm{H}), 7.28-7.26(\mathrm{~m}, 1 \mathrm{H}), 7.26-7.21(\mathrm{~m}, 3 \mathrm{H}), 7.17-7.14(\mathrm{~m}, 2 \mathrm{H}), 4.56(\mathrm{~d}, J=1.3 \mathrm{~Hz}, 1 \mathrm{H})$, $4.49(\mathrm{~d}, J=1.3 \mathrm{~Hz}, 1 \mathrm{H}) .{ }^{13} \mathrm{C}$ NMR $\left(176 \mathrm{MHz}, \mathrm{CDCl}_{3}\right) \delta 191.1,161.0,156.3,142.4,141.0$, 137.4, 136.3, 129.3 (2C), 129.1 (2C), 127.8, 127.5, 127.0 (2C), 126.9 (2C), 125.0, 124.7, 124.1, 119.1, 113.8, 112.2, 47.8, 46.9. The er was determined by $\mathrm{UPC}^{2}$ using a chiral Chiralpack IA column gradient from $100 \% \mathrm{CO}_{2}$ up to $40 \%$; $i-\mathrm{PrOH}$, flow rate $\left.=2.2 \mathrm{~mL} / \mathrm{min}, 1=216 \mathrm{~nm}\right) \mathrm{tR}=$ $3.5 \mathrm{~min}$ (major), $3.3 \mathrm{~min}$ (minor), $(92: 8 \mathrm{er}) .[\alpha]_{\mathrm{D}}^{21}=+395.5(\mathrm{c}=1.0, \mathrm{MeOH}) . \mathrm{HRMS}(\mathrm{ESI}) \mathrm{m} / \mathrm{z}$ $[\mathrm{M}+\mathrm{H}]^{+}$Calcd. for $\mathrm{C}_{25} \mathrm{H}_{19} \mathrm{O}_{2}^{+}$: 351.1380; found: 351.1389. 


\section{Selective transformations of the product 3a}

\subsection{Oxidation of the product 3a to benzofuran-5-carbaldehyde 9}

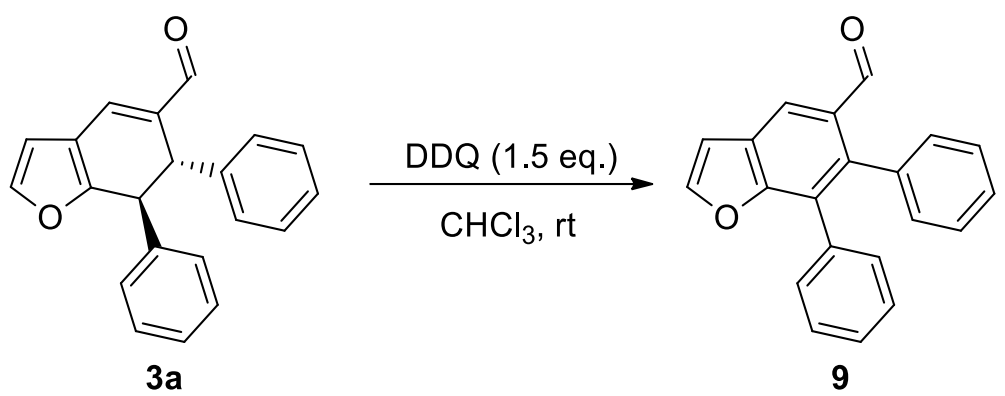

In an ordinary $4 \mathrm{~mL}$ glass vial, equipped with a Teflon-coated magnetic stirring bar the aldehyde 3a (30.1 mg, $0.1 \mathrm{mmol}, 1$ equiv.) was dissolved in $\mathrm{CHCl}_{3}(0.2 \mathrm{~mL})$. Then DDQ (34 mg, 0.15 mmol, 1.5 equiv.) was added and the reaction mixture was stirred at room temperature overnight. After full conversion of the starting material 3a (as confirmed by ${ }^{1} \mathrm{H}$ NMR spectroscopy), the reaction mixture was directly subjected to column chromatography on silica gel (eluent: hexanes/diethyl ether 4:1) to afford pure product 9 in $80 \%$ yield (23.9 mg) as light-yellow oil.

6,7-Diphenylbenzofuran-5-carbaldehyde 9. ${ }^{1} \mathrm{H} \mathrm{NMR}\left(700 \mathrm{MHz}, \mathrm{CDCl}_{3}\right) \delta 9.83(\mathrm{~s}, 1 \mathrm{H}), 8.35$<smiles>O=Cc1cc2ccoc2c(-c2ccccc2)c1-c1ccccc1</smiles>
(s, 1H), $7.70(\mathrm{~d}, J=2.2 \mathrm{~Hz}, 1 \mathrm{H}), 7.29-7.26(\mathrm{~m}, 2 \mathrm{H}), 7.26-7.22(\mathrm{~m}, 4 \mathrm{H})$, $7.21-7.19(\mathrm{~m}, 2 \mathrm{H}), 7.16-7.13(\mathrm{~m}, 2 \mathrm{H}), 6.96(\mathrm{~d}, J=2.2 \mathrm{~Hz}, 1 \mathrm{H}) .{ }^{13} \mathrm{C}$ NMR $\left(176 \mathrm{MHz}, \mathrm{CDCl}_{3}\right) \delta 192.7,156.0,147.7,141.2,136.0,134.1,131.8$ (2C), 131.1, 130.8 (2C), 128.0 (2C), 127.8 (2C), 127.6, 127.5, 127.3, 126.1, 120.6, 107.7. HRMS (ESI) $\mathrm{m} / \mathrm{z}[\mathrm{M}+\mathrm{H}]^{+}$Calcd. for $\mathrm{C}_{21} \mathrm{H}_{15} \mathrm{O}_{2}{ }^{+}$: 299.1067; found: 299.1074 . 


\subsection{Synthesis of diazepine derivative 8}

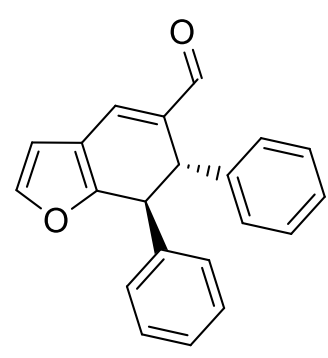

$3 a$<smiles>Nc1ccccc1N</smiles><smiles>CC(C)(C)C(C)(C)C</smiles>

$\mathrm{CH}_{2} \mathrm{Cl}_{2}$

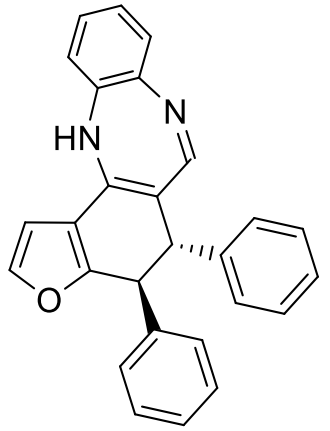

8

In an ordinary $8 \mathrm{~mL}$ glass vial, equipped with a Teflon-coated magnetic stirring bar the aldehyde 3a (30.1 mg, $0.1 \mathrm{mmol}, 1$ equiv.) was dissolved in $\mathrm{MeOH} / \mathrm{CH}_{2} \mathrm{Cl}_{2} 3: 1 \mathrm{v} / \mathrm{v}(1 \mathrm{~mL})$. Then $o$-phenylenediamine $\left(10.8 \mathrm{mg}, 0.1 \mathrm{mmol}, 1\right.$ equiv.) and $\mathrm{CeCl}_{3} \cdot 7 \mathrm{H}_{2} \mathrm{O}(37.2 \mathrm{mg}, 0.1 \mathrm{mmol}, 1$ equiv.) were added and the reaction mixture was stirred at $50{ }^{\circ} \mathrm{C}$ for 20 hours. After full conversion of the starting material 3a (as confirmed by TLC analysis), the reaction mixture was diluted with $\mathrm{CH}_{2} \mathrm{Cl}_{2}(10 \mathrm{~mL})$ and washed with water $(2 \times 5 \mathrm{~mL})$, dried over anhydrous sodium sulfate, filtered and concentrated under reduced pressure. The resulting solid was subjected to column chromatography on silica gel (eluent: $\mathrm{CH}_{2} \mathrm{Cl}_{2}$ ) to afford pure product 8 (>20:1 dr) in $62 \%$ yield $(24.0 \mathrm{mg})$ as light-yellow oil.

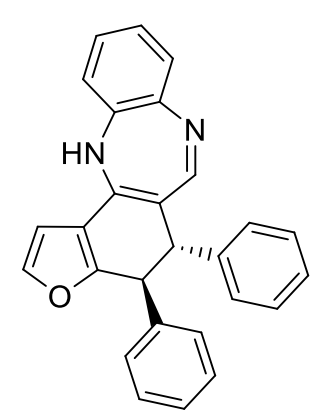

(4S,5S)-4,5-Diphenyl-5,12-dihydro-4H-benzo[b]benzofuro[4,5-

$\boldsymbol{e}][\mathbf{1 , 4 ]}]$ diazepine 8. ${ }^{1} \mathrm{H}$ NMR $\left(700 \mathrm{MHz}, \mathrm{DMSO}-d_{6}\right) \delta 12.57(\mathrm{~s}, 1 \mathrm{H}), 7.72(\mathrm{~s}$, $1 \mathrm{H}), 7.65(\mathrm{~d}, J=1.9 \mathrm{~Hz}, 1 \mathrm{H}), 7.45(\mathrm{~d}, J=8.0 \mathrm{~Hz}, 1 \mathrm{H}), 7.43-7.39(\mathrm{~m}, 2 \mathrm{H})$, $7.37(\mathrm{~d}, J=8.0 \mathrm{~Hz}, 1 \mathrm{H}), 7.30(\mathrm{t}, J=7.7 \mathrm{~Hz}, 2 \mathrm{H}), 7.25(\mathrm{t}, J=7.7 \mathrm{~Hz}, 2 \mathrm{H})$, $7.24-7.20(\mathrm{~m}, 1 \mathrm{H}), 7.20-7.15(\mathrm{~m}, 3 \mathrm{H}), 7.14-7.03(\mathrm{~m}, 2 \mathrm{H}), 6.79-6.75$ $(\mathrm{m}, 1 \mathrm{H}), 4.93(\mathrm{~s}, 1 \mathrm{H}), 4.33(\mathrm{~s}, 1 \mathrm{H}) .{ }^{13} \mathrm{C}$ NMR (176 MHz, DMSO-d $) \delta 152.6$, 151.5, 143.9, 143.4, 142.1, 141.6, 134.7, 128.8 (2C), 128.5 (2C), 127.1 (2C), 127.0, 126.8, 126.7 (2C), 124.7, 122.5, 121.6, 121.3, 118.6, 118.0, 110.7, 108.6, 49.8, 47.1. $[\alpha]_{\mathrm{D}}^{23}=+249.9(\mathrm{c}=1.0$, $\mathrm{MeOH}$ ). HRMS (ESI) m/z [M+H] $]^{+}$Calcd. for $\mathrm{C}_{27} \mathrm{H}_{21} \mathrm{~N}_{2} \mathrm{O}^{+}$: 389.1649; found: 389.1644. 


\subsection{Selective reduction of aldehyde $3 \mathbf{a}$}

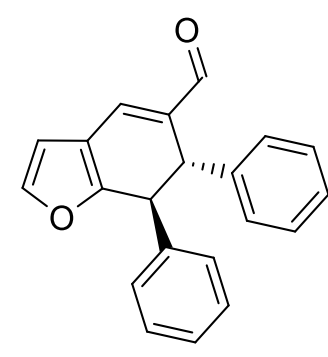

$3 \mathbf{a}$

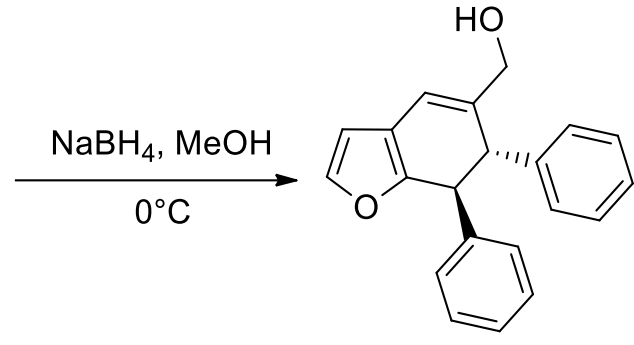

7

In an ordinary $4 \mathrm{~mL}$ glass vial equipped with a magnetic stirring bar the aldehyde 3a $(30.1 \mathrm{mg}$, $0.1 \mathrm{mmol}, 1$ equiv.) was dissolved in $\mathrm{CH}_{2} \mathrm{Cl}_{2}(0.2 \mathrm{~mL})$. Then $\mathrm{MeOH}(0.1 \mathrm{~mL})$ and $\mathrm{NaBH}_{4}(15.2$ $\mathrm{mg}, 0.4 \mathrm{mmol}, 4$ equiv.) were added and the reaction mixture was stirred in room temperature for $30 \mathrm{~min}$. Then the reaction mixture was directly subjected to column chromatography on silica gel (eluent: hexanes/ethyl acetate $4: 1)$ to afford pure product 7 in $78 \%$ yield $(23.6 \mathrm{mg}$ ) as lightyellow oil.

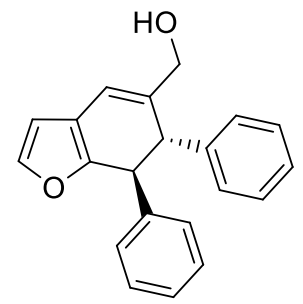

((6S,7S)-6,7-Diphenyl-6,7-dihydrobenzofuran-5-yl)methanol 7. ${ }^{1} \mathrm{H}$ NMR $\left(700 \mathrm{MHz}, \mathrm{CDCl}_{3}\right) \delta 7.30-7.26(\mathrm{~m}, 5 \mathrm{H}), 7.26-7.21(\mathrm{~m}, 4 \mathrm{H}), 7.14-7.10$ $(\mathrm{m}, 2 \mathrm{H}), 6.60(\mathrm{dt}, J=1.5,0.8 \mathrm{~Hz}, 1 \mathrm{H}), 6.43(\mathrm{dd}, J=1.9,0.6 \mathrm{~Hz}, 1 \mathrm{H}), 4.18(\mathrm{~d}$, $J=2.3 \mathrm{~Hz}, 1 \mathrm{H}), 4.05-4.01(\mathrm{~m}, 1 \mathrm{H}), 3.97(\mathrm{~d}, J=13.9 \mathrm{~Hz}, 1 \mathrm{H}), 3.80(\mathrm{~d}, J=$ $2.4 \mathrm{~Hz}, 1 \mathrm{H}) .{ }^{13} \mathrm{C} \mathrm{NMR}\left(176 \mathrm{MHz}, \mathrm{CDCl}_{3}\right) \delta 151.5,142.8,142.7,142.6$, 135.7, 129.1 (2C), 129.0 (2C), 127.5 (2C), 127.3, 127.2, 127.1 (2C), 117.7, 117.2, 108.2, 65.3, 52.8, 48.2. HRMS (ESI) m/z [M+H] ${ }^{+}$Calcd. for $\mathrm{C}_{21} \mathrm{H}_{19} \mathrm{O}_{2}{ }^{+}$: 303.1380; found: 303.1384. 


\subsection{Enantioselective synthesis of (6S,7S)-6,7-diphenyl-6,7-dihydrobenzofuran-5- carbaldehyde 3a on a $1 \mathrm{mmol}$ scale}

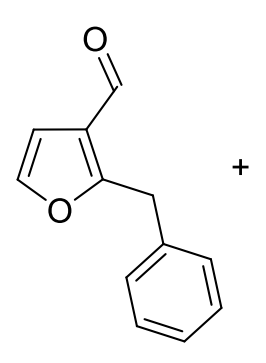

$1 \mathrm{a}$

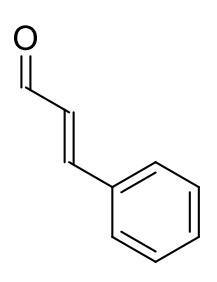

$2 a$
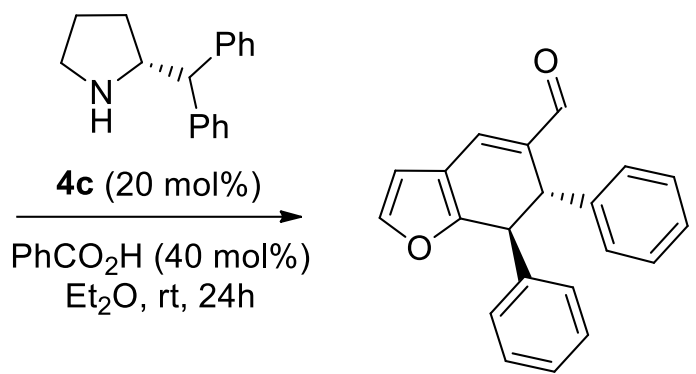

$3 a$

In an ordinary $8 \mathrm{~mL}$ glass vial equipped with a magnetic stirring bar $\alpha, \beta$-unsaturated aldehyde $2 \mathbf{a}$ (158.0 mg, $1.2 \mathrm{mmol}, 1.2$ equiv.) and heteroaromatic aldehyde $1 \mathbf{a}(186.0 \mathrm{mg}, 1.0 \mathrm{mmol}, 1.0$ equiv.) were dissolved in $\mathrm{Et}_{2} \mathrm{O}(4 \mathrm{~mL})$ and catalyst $4 \mathbf{c}(47.0 \mathrm{mg}, 0.2 \mathrm{mmol}, 0.2$ equiv.) and benzoic acid ( $49.0 \mathrm{mg}, 0.4 \mathrm{mmol}, 0.4$ equiv.) were added and the reaction mixture was stirred in room temperature for indicated time. The progress of the reaction was controlled by ${ }^{1} \mathrm{H}$ NMR spectroscopy. After full conversion of the starting material 1a, the reaction mixture was directly subjected to column chromatography on silica gel (hexanes : diethyl ether 85:15) to afford pure product 3a as single diastereoisomer in $91 \%$ yield $(273.0 \mathrm{mg})$ as light-yellow solid. Spectral data were in accordance with the previously reported on page S3. 


\section{Crystal and X-ray data for 3a}

The crystal structure of the compound (6S,7S)-6,7-diphenyl-6,7-dihydrobenzofuran-5carbaldehyde 3a, $\mathrm{C}_{21} \mathrm{H}_{16} \mathrm{O}_{2}$, was established by single-crystal X-ray diffraction at $100 \mathrm{~K}$. The compound crystallizes in the non-centrosymmetric orthorhombic space group $P 2{ }_{1} 2_{1} 2_{1}(Z=4)$ and the crystal structure consists of one crystallographically independent formula unit in the unit cell (Figure 1).

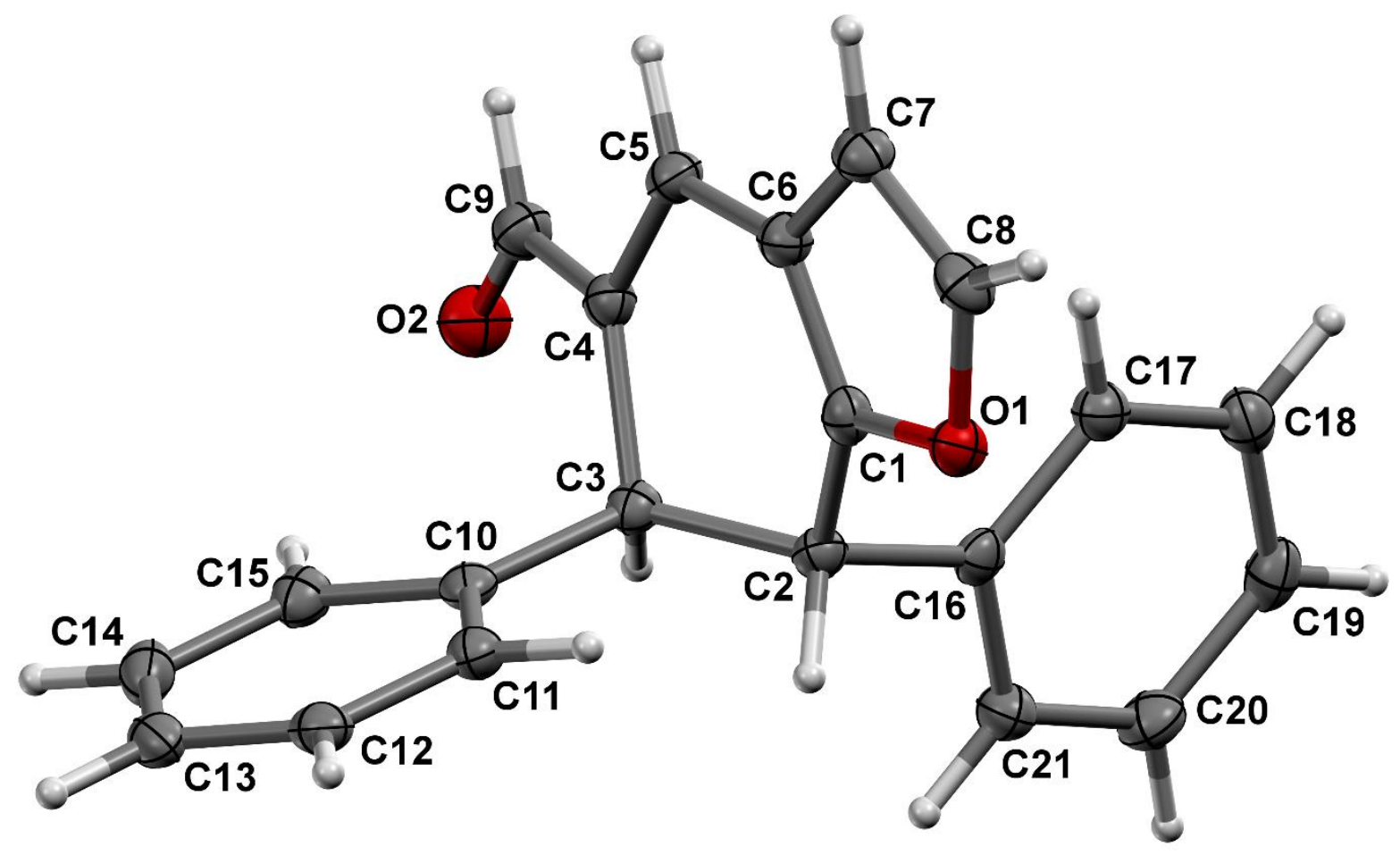

Figure 1. The molecular structure of the compound 3a at $100 \mathrm{~K}$, with the atom labeling scheme, showing $50 \%$ probability displacement ellipsoids. Hydrogen atoms are drawn with an arbitrary radius.

Single crystal X-ray diffraction data were collected at $100 \mathrm{~K}$ by the $\omega$-scan technique using a RIGAKU XtaLAB Synergy, Dualflex, Pilatus 300K diffractometer ${ }^{3}$ with PhotonJet micro-focus $\mathrm{X}$-ray Source $\mathrm{Cu}-\mathrm{K} \alpha(\lambda=1.54184 \AA)$. Data collection, cell refinement, data reduction and absorption correction were performed using CrysAlis PRO software. ${ }^{3}$ The crystal structure was solved by using direct methods with the SHELXT 2018/2 program. ${ }^{4}$ Atomic scattering factors were taken from the International Tables for X-ray Crystallography. Positional parameters of non-H-atoms were refined by a full-matrix least-squares method on $\mathrm{F}^{2}$ with anisotropic thermal 
parameters by using the SHELXL 2018/3 program. ${ }^{5}$ All hydrogen atoms were found from the difference Fourier maps and for further calculations they were positioned geometrically in calculated positions $(\mathrm{C}-\mathrm{H}=0.95-1.00 \AA)$ and constrained to ride on their parent atoms with isotropic displacement parameters set to 1.2 times the $\mathrm{U}_{\mathrm{eq}}$ of the parent atom.

(6S,7S)-6,7-Diphenyl-6,7-dihydrobenzofuran-5-carbaldehyde 3a: Formula $\quad \mathrm{C}_{21} \mathrm{H}_{16} \mathrm{O}_{2}$, orthorhombic, space group $P 2{ }_{1} 2_{1} 2_{1}, Z=4$, unit cell constants $a=8.12398(4), b=10.30996(6), c$ $=17.96578(9) \AA, V=1504.776(14) \AA^{3}$. The integration of the data yielded a total of 41425 reflections with $\theta$ angles in the range of 4.92 to $66.59^{\circ}$, of which 2662 were independent $\left(R_{\text {int }}=\right.$ $2.92 \%$ ), and 2642 were greater than $2 \sigma\left(\mathrm{F}^{2}\right)$. The final anisotropic full-matrix least-squares refinement on $\mathrm{F}^{2}$ with 209 parameters converged at $\mathrm{R}_{1}=2.34 \%$ and $\mathrm{wR}_{2}=5.92 \%$ for all data. The largest peak in the final difference electron density synthesis was $0.162 \mathrm{e} \AA^{-3}$ and the largest hole was -0.144 e $\AA^{-3}$. The goodness-of-fit was 1.055 . The absolute configuration was unambiguously established from anomalous scattering, by calculating the $x$ Flack parameter ${ }^{6}$ of 0.00(3) using 1099 quotients.

CCDC 2103952 contains the supplementary crystallographic data for this paper. These data can be obtained free of charge from The Cambridge Crystallographic Data Centre via www.ccdc.cam.ac.uk/structures

\section{References}

(3) Rigaku OD. CrysAlis PRO. Rigaku Oxford Diffraction Ltd, Yarnton, Oxfordshire, England, 2019.

(4) Sheldrick, G. M. SHELXT - integrated space-group and crystal-structure determination. Acta Cryst. 2015, A71, 3-8.

(5) Sheldrick, G. M. Crystal structure refinement with SHELXL. Acta Cryst. 2015, C71, 3-8.

(6) Parsons, S.; Flack, H. D.; Wagner, T. Use of intensity quotients and differences in absolute structure refinement. Acta Cryst. 2013, B69, 249-259. 


\section{Non-linear effect study}

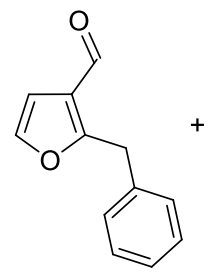

$1 \mathrm{a}$<smiles>O=C/C=C/c1ccccc1</smiles>

$2 a$

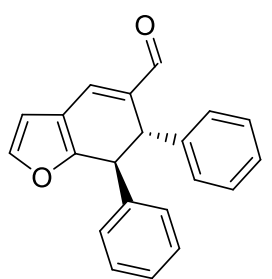

$3 a$<smiles>c1ccc(C(c2ccccc2)C2CCCN2)cc1</smiles>

(R)-4c (S)-4c

The experiments for non-linear effect study were carried out according to the procedure of the synthesis of 3a. The catalyst $\mathbf{4 c}$ mixtures with different ee values were prepared by mixing $(S)-\mathbf{4 c}$ and $(R)-\mathbf{4} \mathbf{c}$ in appropriate ratios (with the $(R)-\mathbf{4} \mathbf{c}$ being the major). The ee value of product $\mathbf{3 a}$ was determined by $\mathrm{UPC}^{2}$, which indicated a non-linear relationship between ee values of products $\mathbf{3 a}$ and amine catalyst $\mathbf{4 c}$, as shown in the figure below.

\section{Non-linear effect studies}

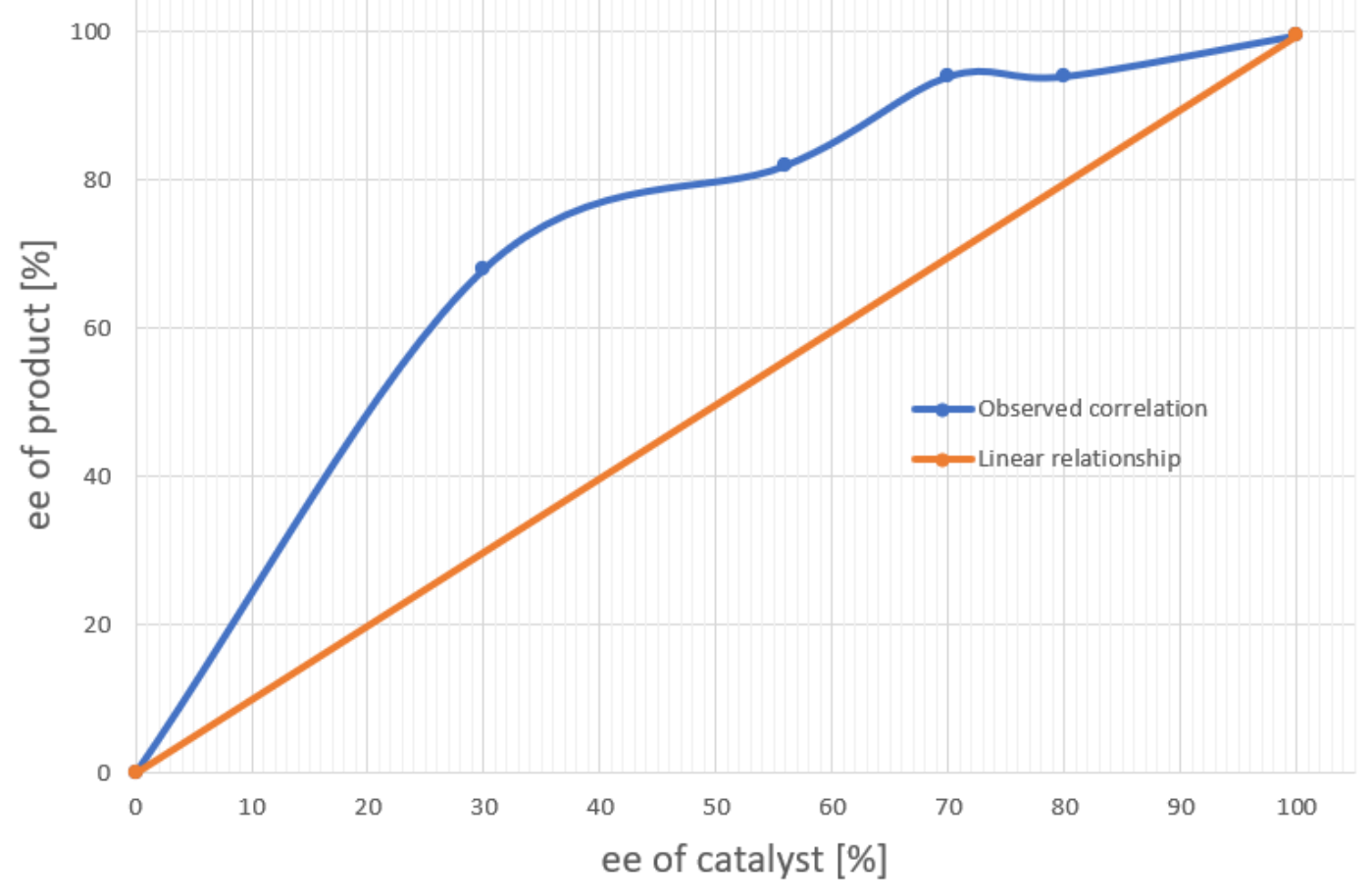




\section{NMR Data}

(6S,7S)-6,7-Diphenyl-6,7-dihydrobenzofuran-5-carbaldehyde 3a

${ }^{1} \mathrm{H}$ NMR (700 MHz, $\left.\mathrm{CDCl}_{3}\right)$

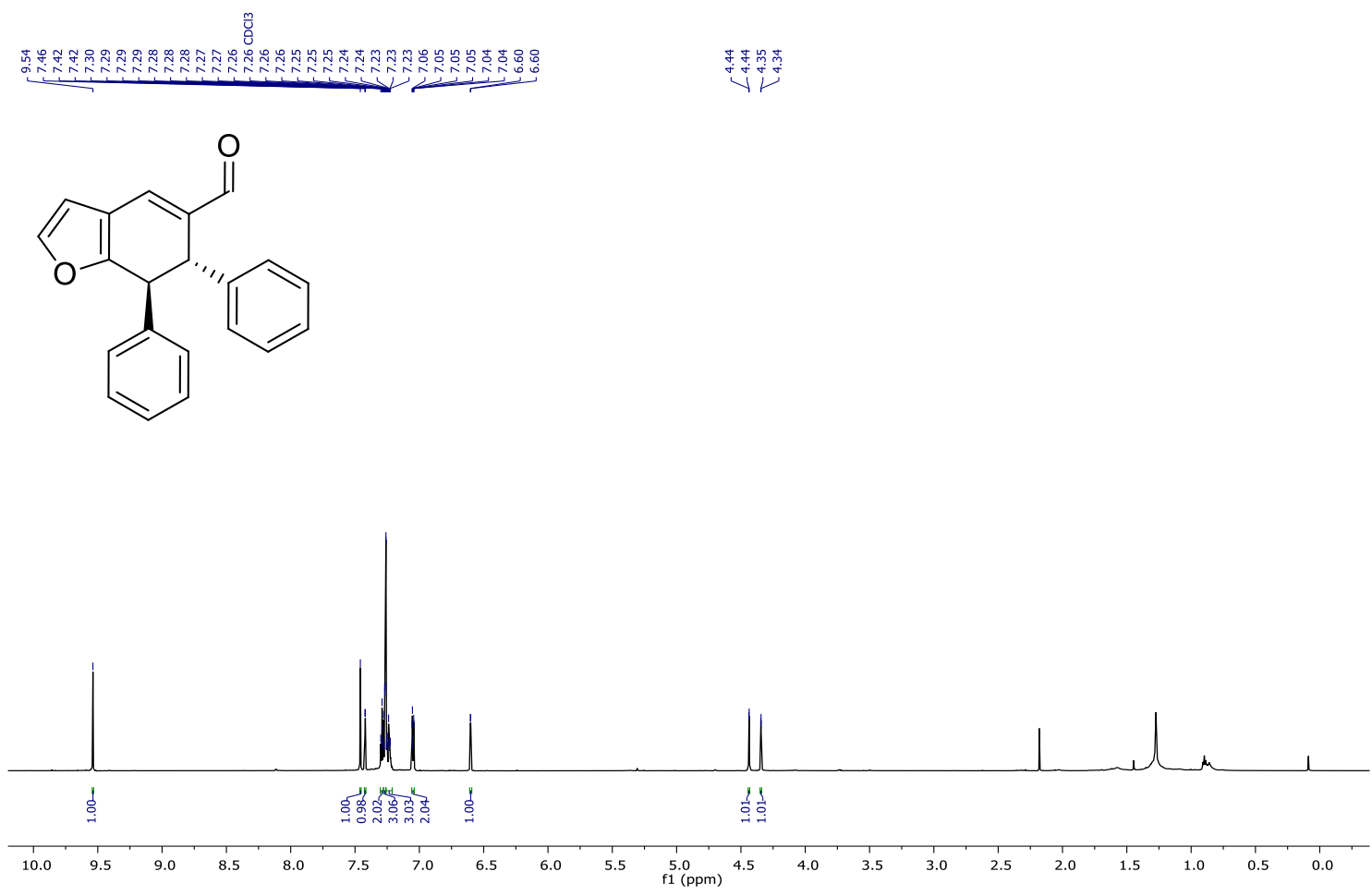

${ }^{13} \mathrm{C}$ NMR (176 MHz, CDCl 3$)$
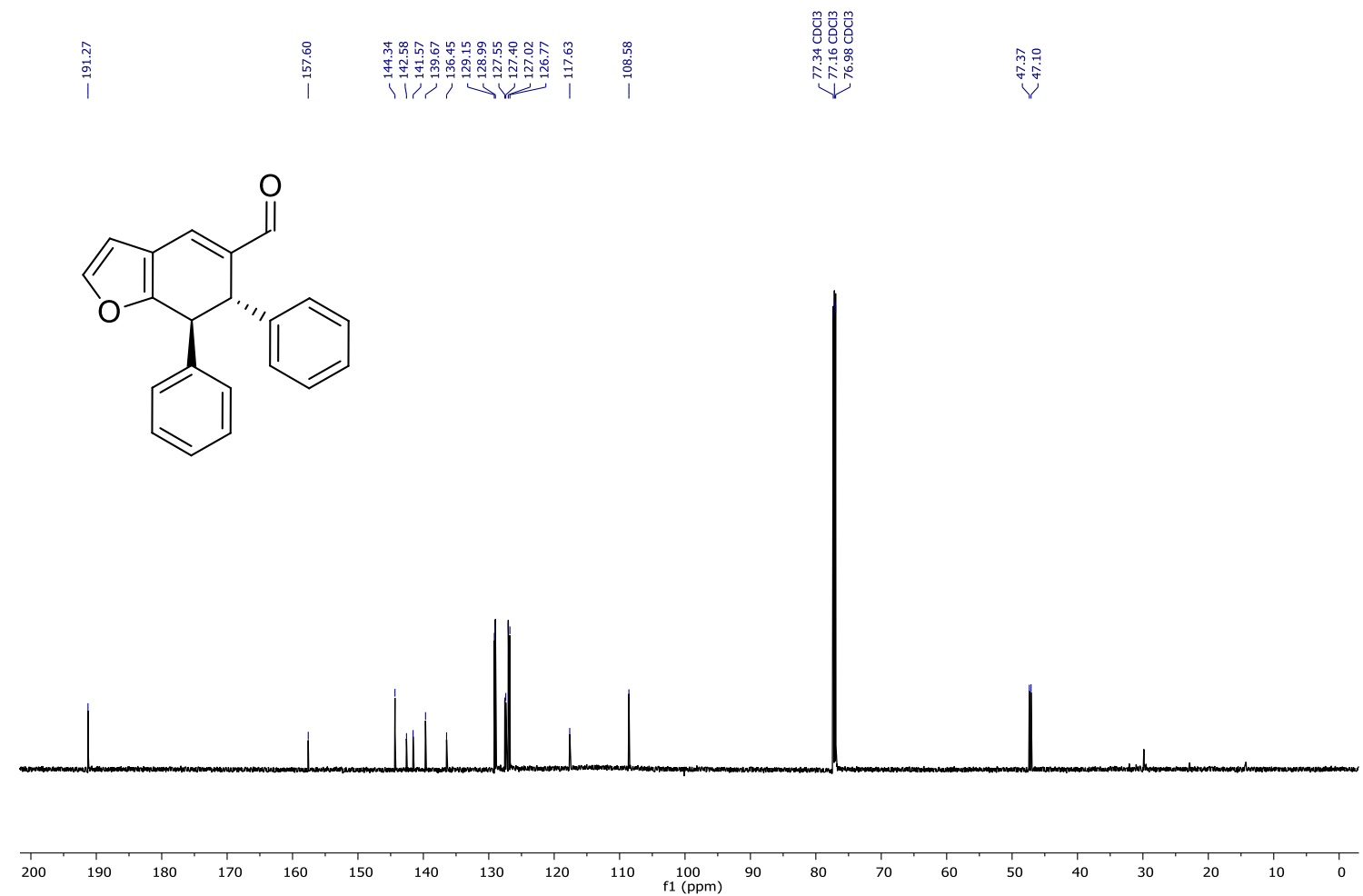
(6S,7S)-6-(4-Nitrophenyl)-7-phenyl-6,7-dihydrobenzofuran-5-carbaldehyde 3b ${ }^{1} \mathrm{H}$ NMR (700 MHz, $\left.\mathrm{CDCl}_{3}\right)$

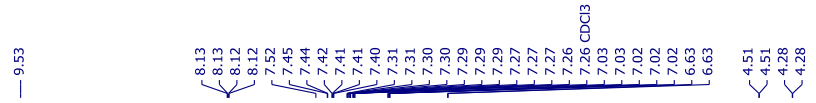<smiles>O=CC1=Cc2ccoc2C(c2ccccc2)C1c1ccc([N+](=O)[O-])cc1</smiles>

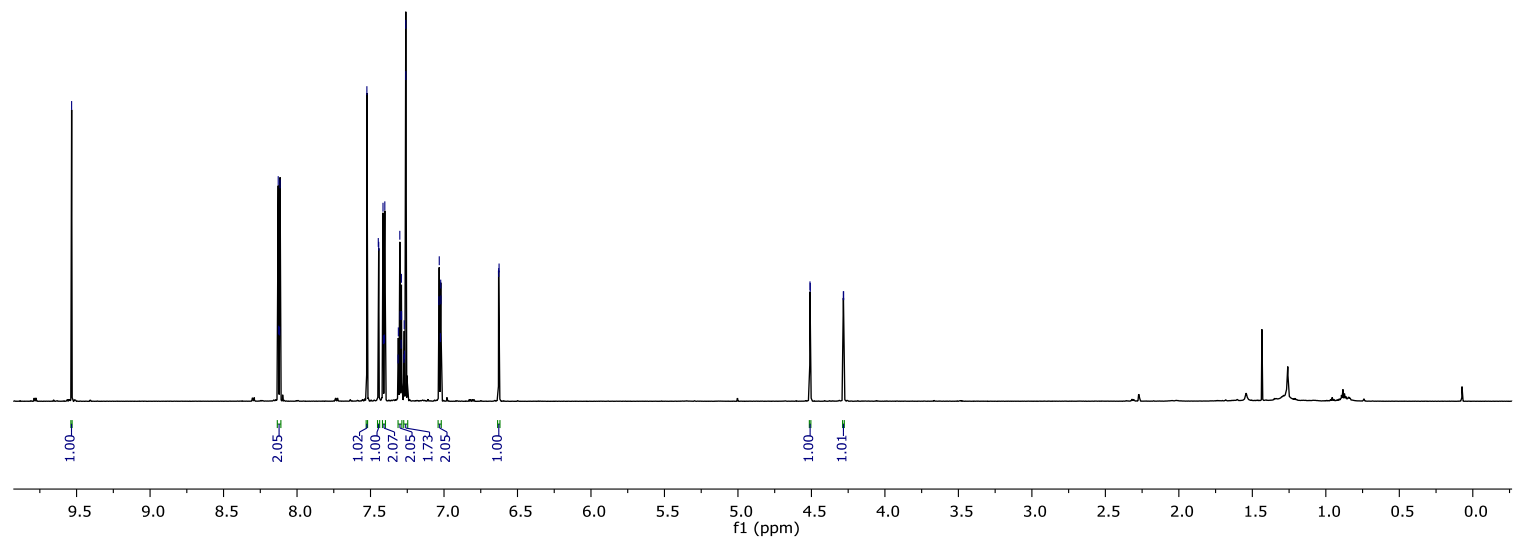

${ }^{13} \mathrm{C}$ NMR (176 MHz, $\left.\mathrm{CDCl}_{3}\right)$

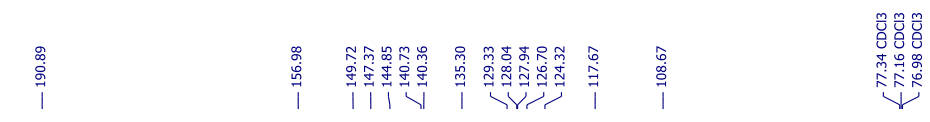<smiles>O=CC1=Cc2ccoc2C(c2ccccc2)C1c1ccc([N+](=O)[O-])cc1</smiles>

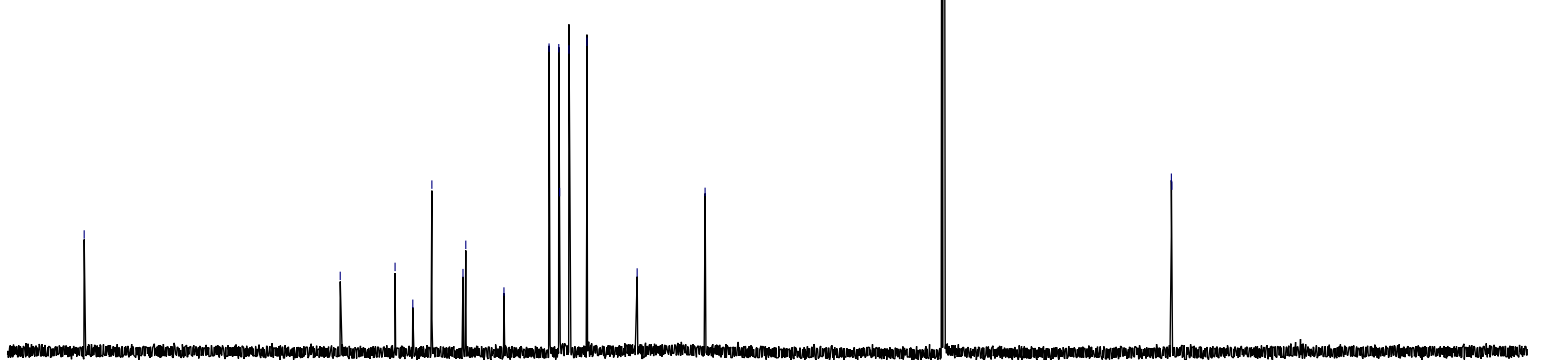

200

$150+140$

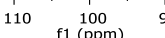


(6S,7S)-6-(4-Chlorophenyl)-7-phenyl-6,7-dihydrobenzofuran-5-carbaldehyde 3c ${ }^{1} \mathrm{H}$ NMR (700 $\left.\mathrm{MHz}, \mathrm{CDCl}_{3}\right)$<smiles>C#CC1=Cc2ccoc2[C@@H](c2ccccc2)[C@H]1c1ccc(Cl)cc1</smiles>
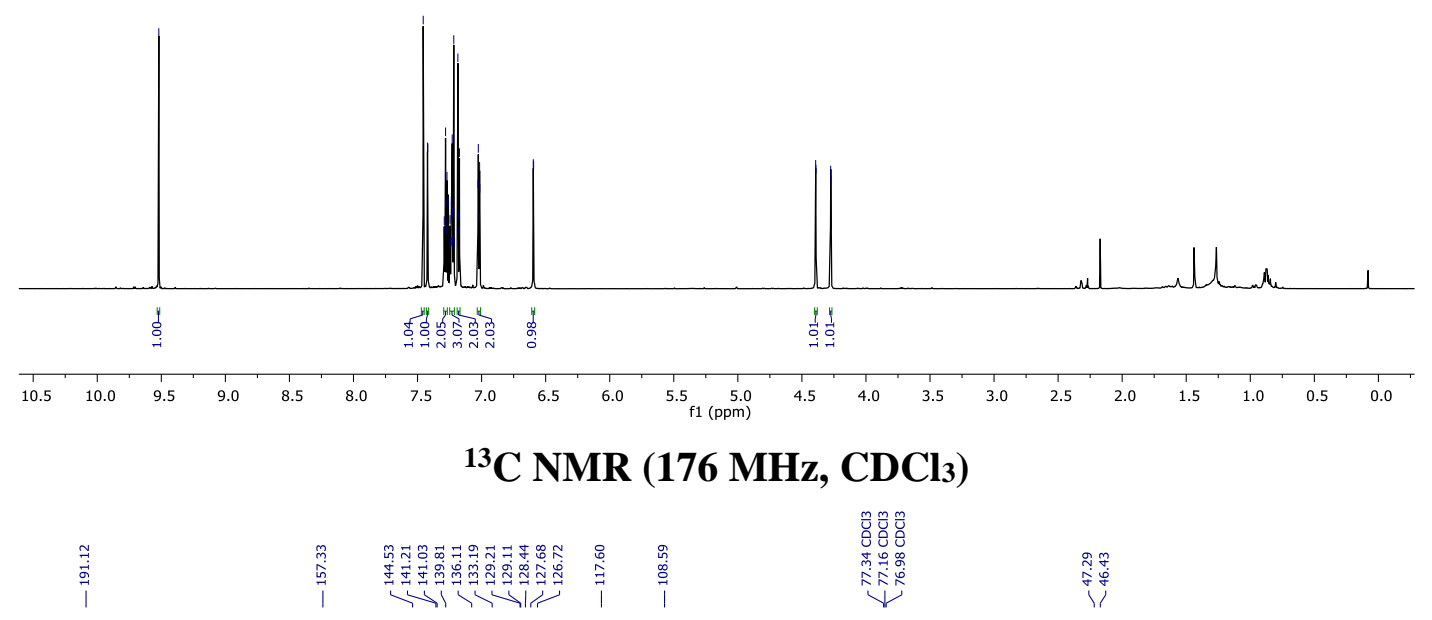<smiles>O=CC1=Cc2ccoc2[C@H](c2ccccc2)[C@H]1c1ccc(Cl)cc1</smiles>
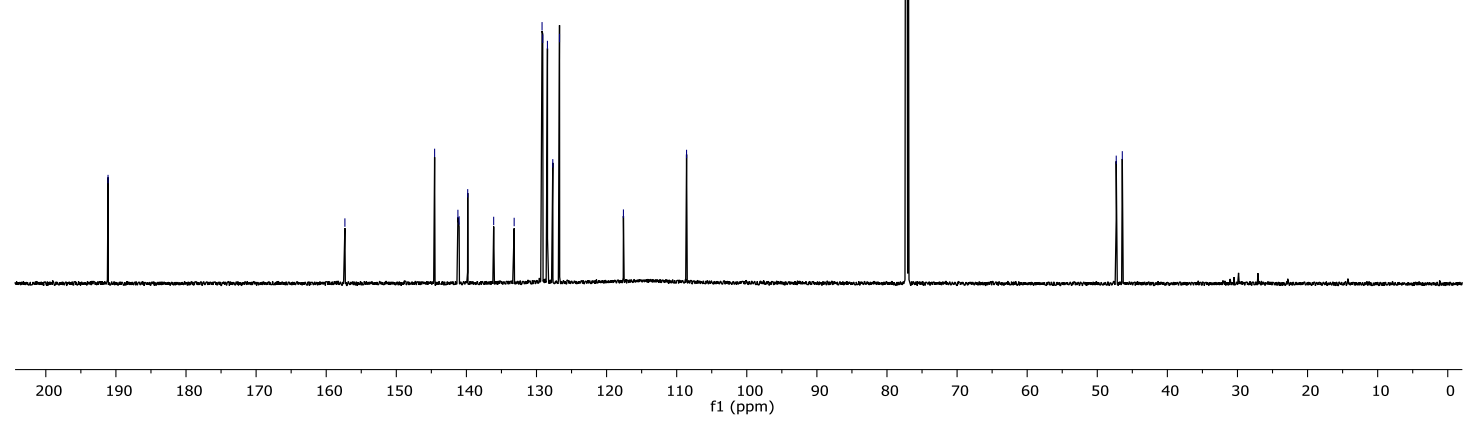
(6S,7S)-7-Phenyl-6-(p-tolyl)-6,7-dihydrobenzofuran-5-carbaldehyde 3d

${ }^{1} \mathrm{H}$ NMR (700 MHz, $\left.\mathrm{CDCl}_{3}\right)$

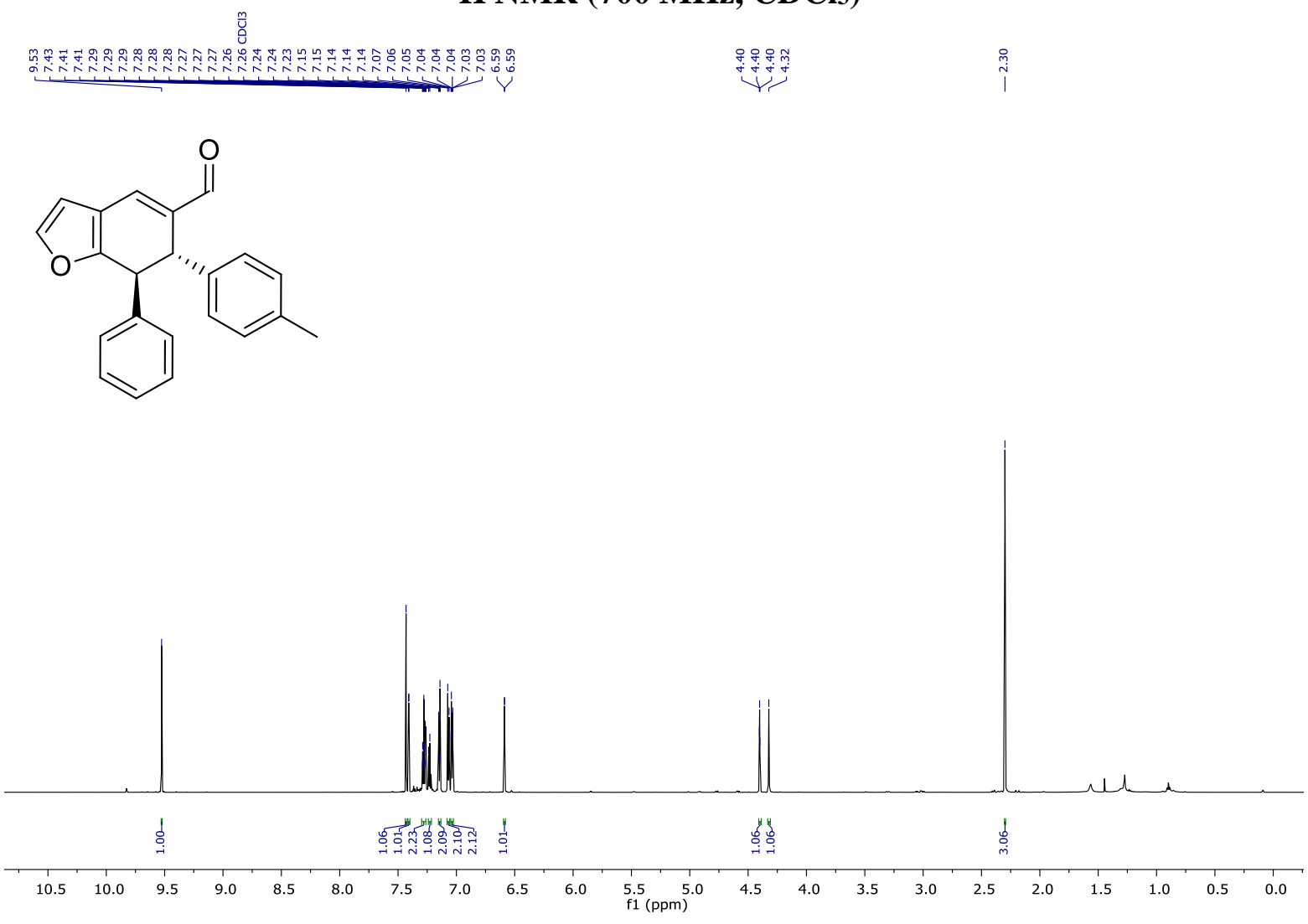

${ }^{13} \mathrm{C}$ NMR (176 MHz, $\left.\mathrm{CDCl}_{3}\right)$

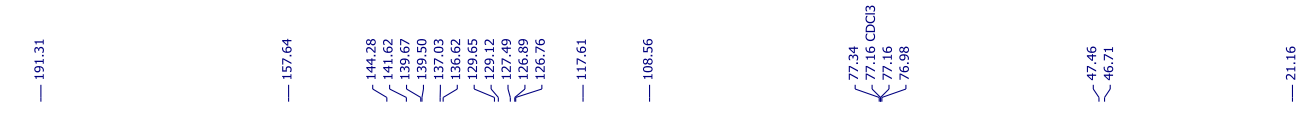<smiles>Cc1ccc([C@H]2C(C=O)=Cc3ccoc3C2c2ccccc2)cc1</smiles>

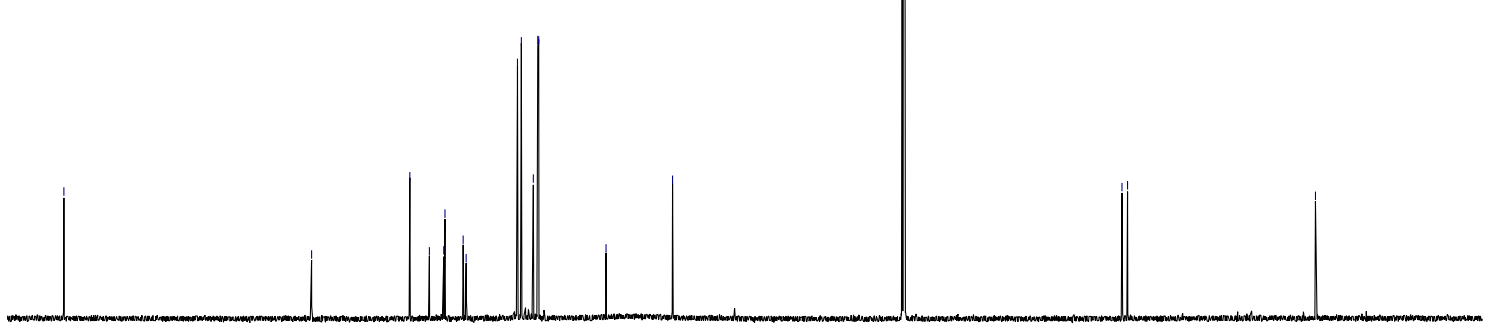


(6S,7S)-6-(4-Methoxyphenyl)-7-phenyl-6,7-dihydrobenzofuran-5-carbaldehyde 3e ${ }^{1} \mathrm{H}$ NMR (700 MHz, $\left.\mathrm{CDCl}_{3}\right)$

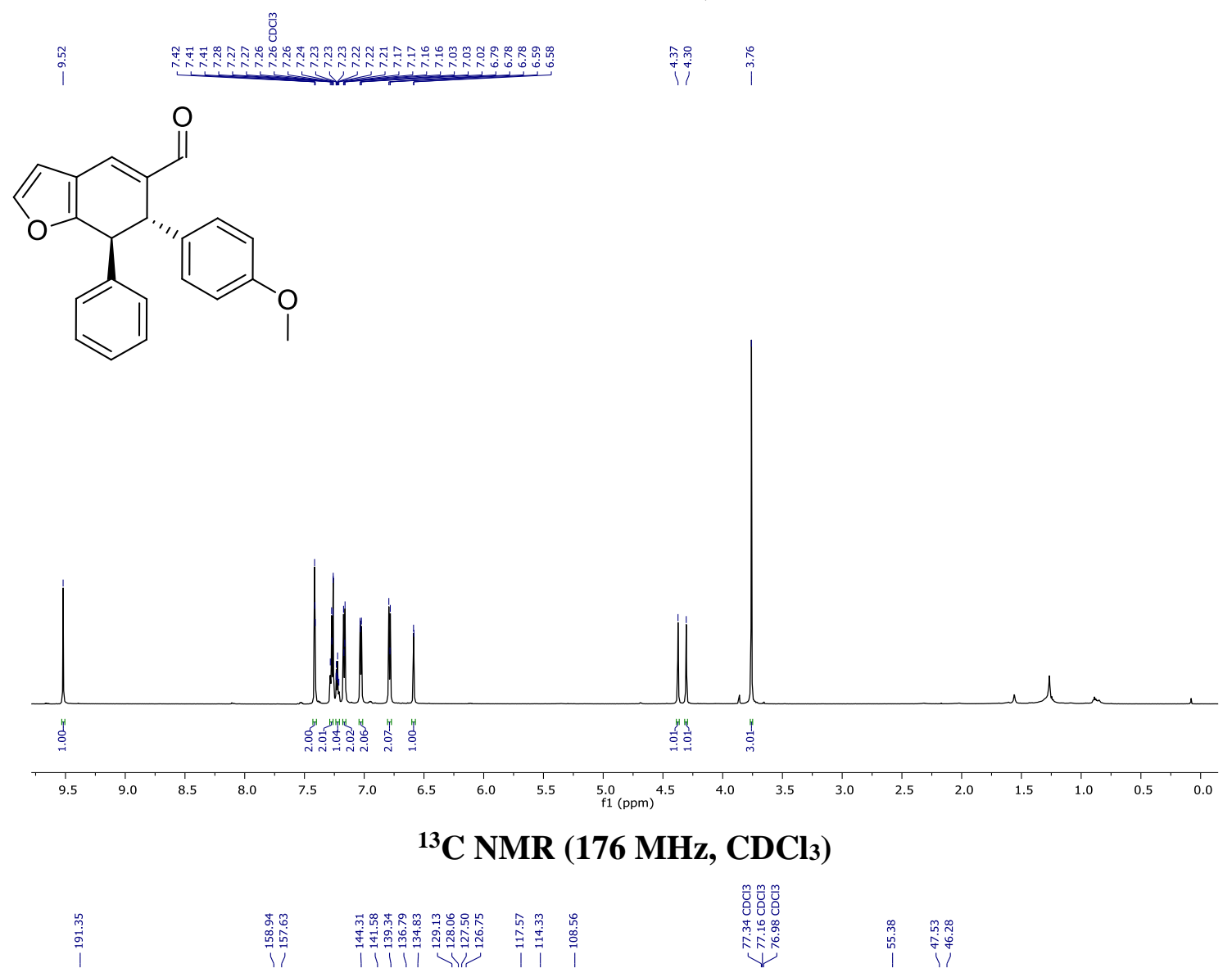<smiles>COc1ccc([C@H]2C(C=O)=Cc3ccoc3C2c2ccccc2)cc1</smiles>
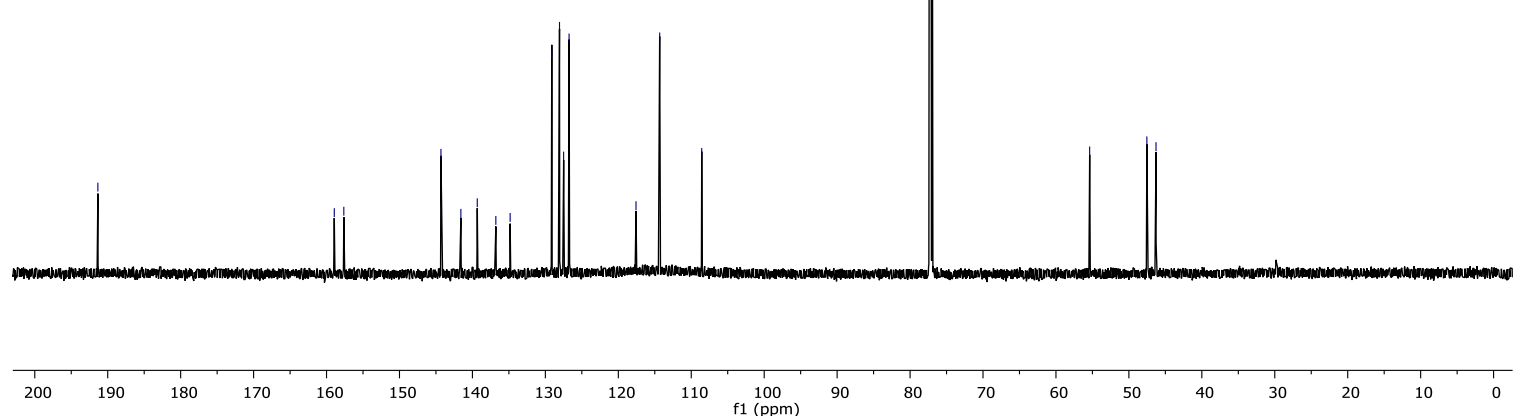
(6S,7S)-6-(3-Methoxyphenyl)-7-phenyl-6,7-dihydrobenzofuran-5-carbaldehyde 3f ${ }^{1} \mathrm{H}$ NMR (700 MHz, $\left.\mathrm{CDCl}_{3}\right)$
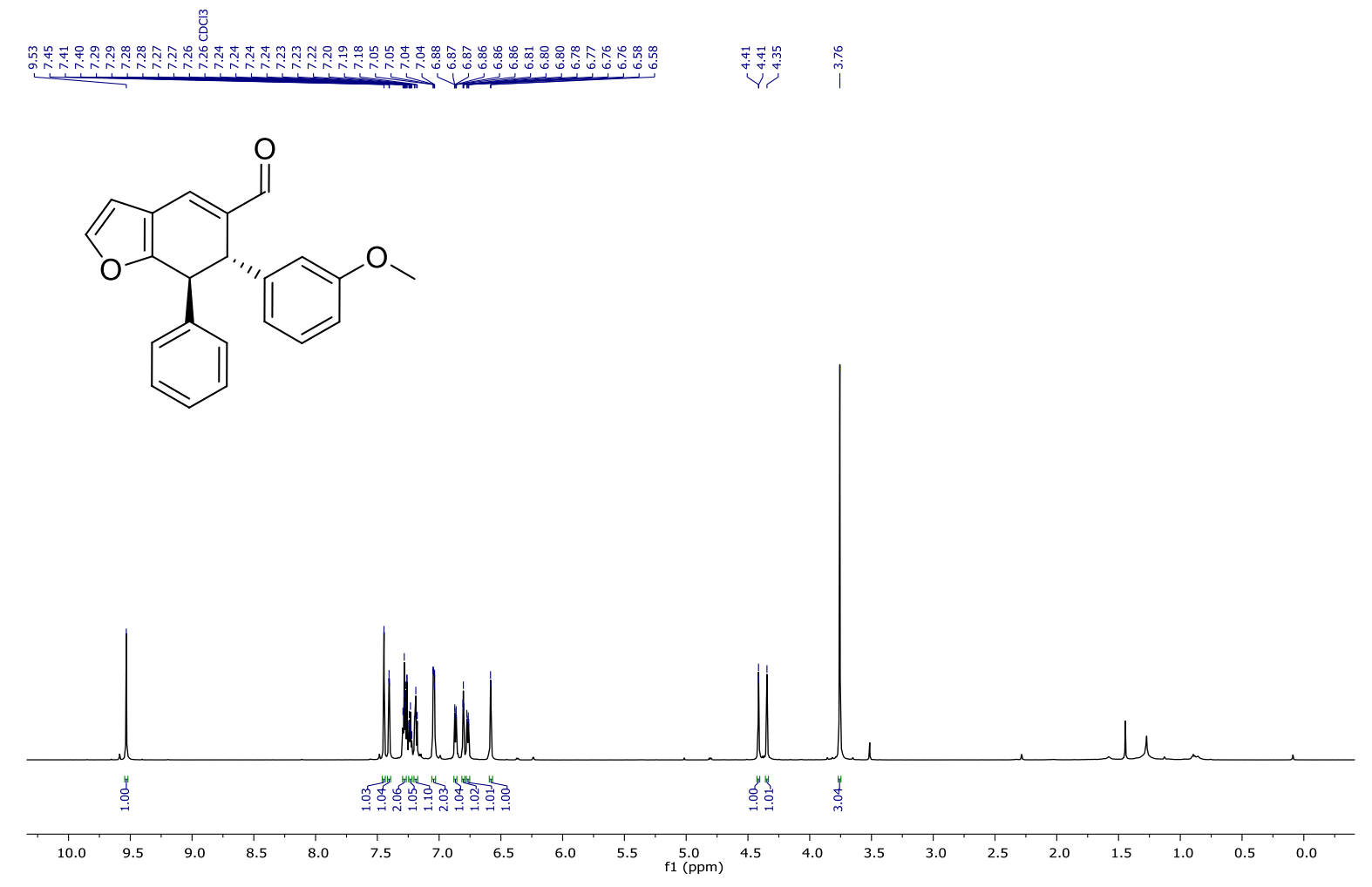

${ }^{13} \mathrm{C}$ NMR (176 MHz, $\left.\mathrm{CDCl}_{3}\right)$
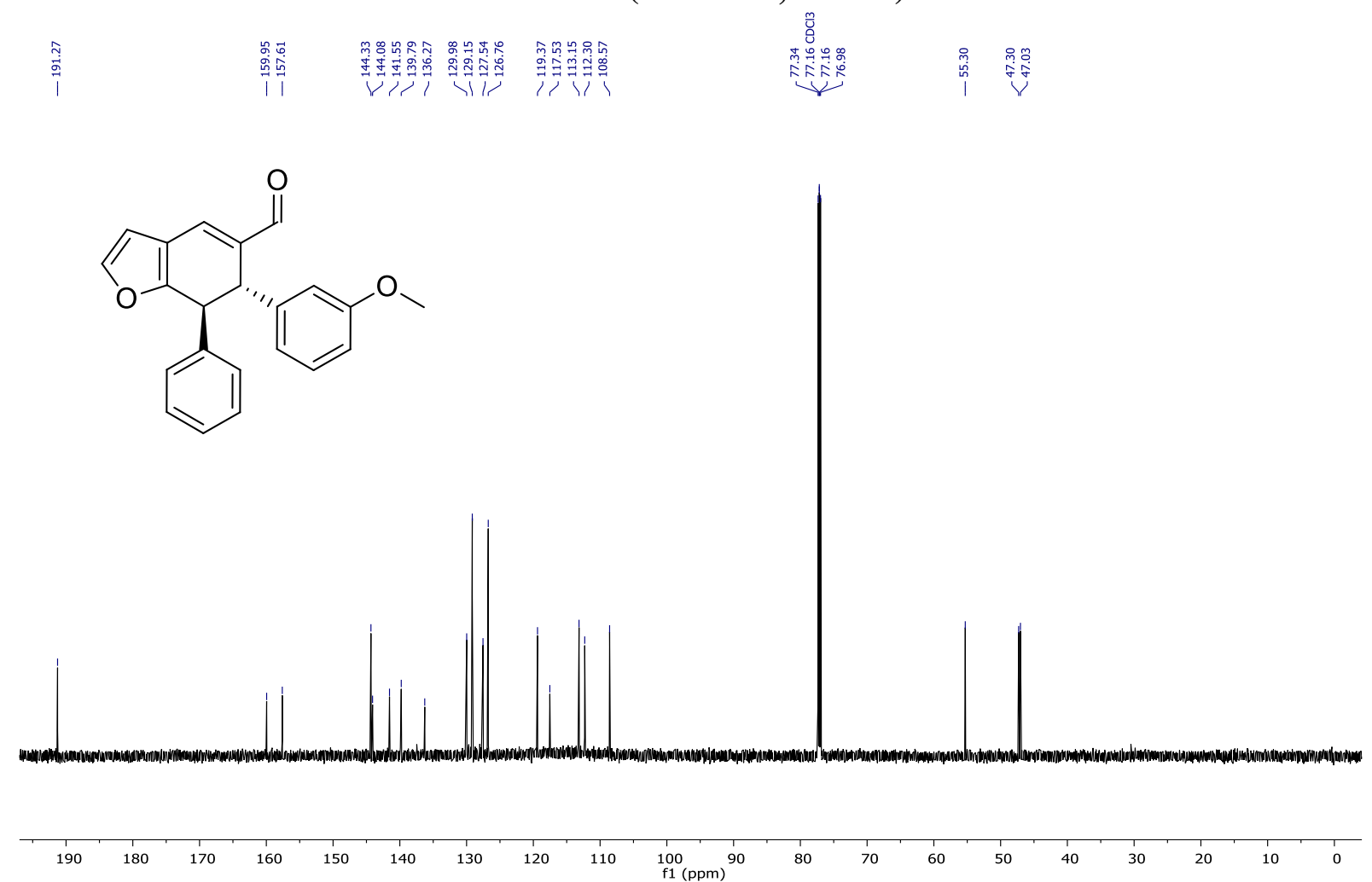
(6S,7S)-6-(2-Methoxyphenyl)-7-phenyl-6,7-dihydrobenzofuran-5-carbaldehyde 3g ${ }^{1} \mathrm{H}$ NMR $\left(700 \mathrm{MHz}, \mathrm{CDCl}_{3}\right)$

范

$\underbrace{.04}$<smiles>COc1ccccc1[C@@H]1C(C=O)=Cc2ccoc2[C@H]1c1ccccc1</smiles>

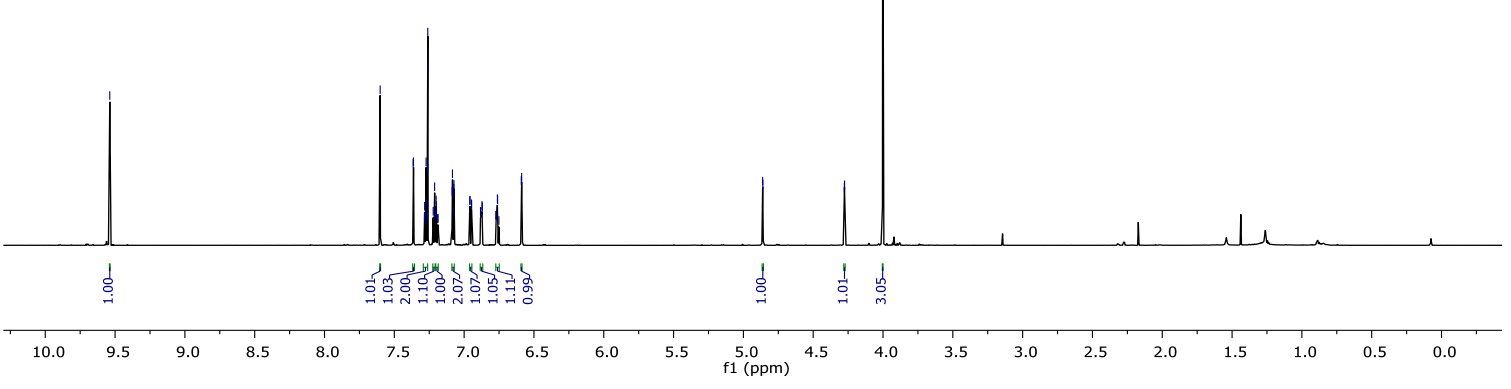

${ }^{13} \mathrm{C}$ NMR (176 MHz, $\left.\mathrm{CDCl}_{3}\right)$

I

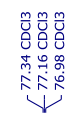
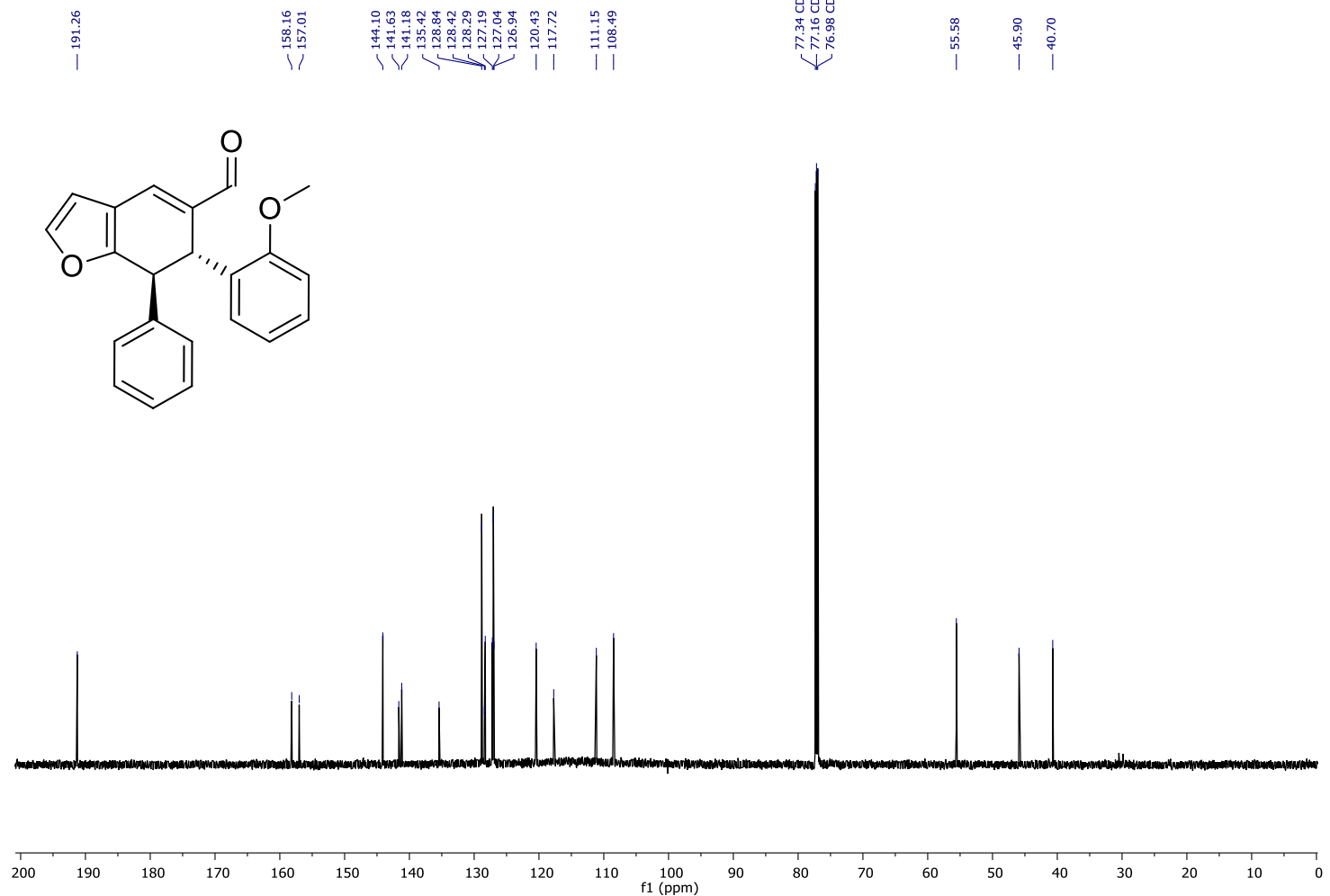

S23 
(6R,7S)-6-(2,4-Dichlorophenyl)-7-phenyl-6,7-dihydrobenzofuran-5-carbaldehyde $3 \mathrm{~h}$ ${ }^{1} \mathrm{H}$ NMR (700 MHz, $\left.\mathrm{CDCl}_{3}\right)$

范

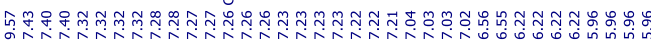

Y V V

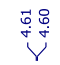<smiles>O=CC1=Cc2ccoc2[C@H](c2ccccc2)[C@H]1c1ccc(Cl)cc1Cl</smiles>

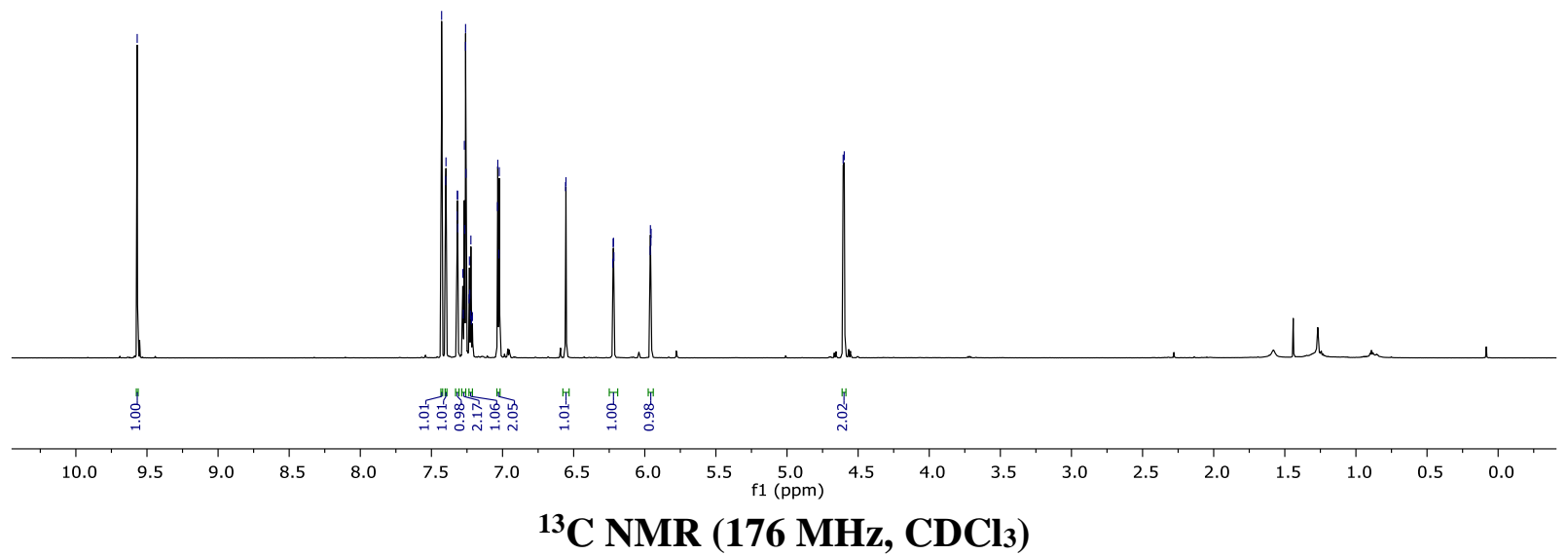

$\vec{\infty}$
$\stackrel{0}{0}$
$\stackrel{0}{1}$
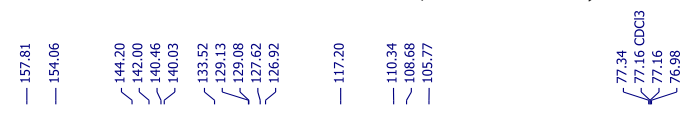

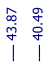<smiles>O=CC1=Cc2ccoc2[C@H](c2ccccc2)[C@H]1c1ccc(Cl)cc1Cl</smiles>

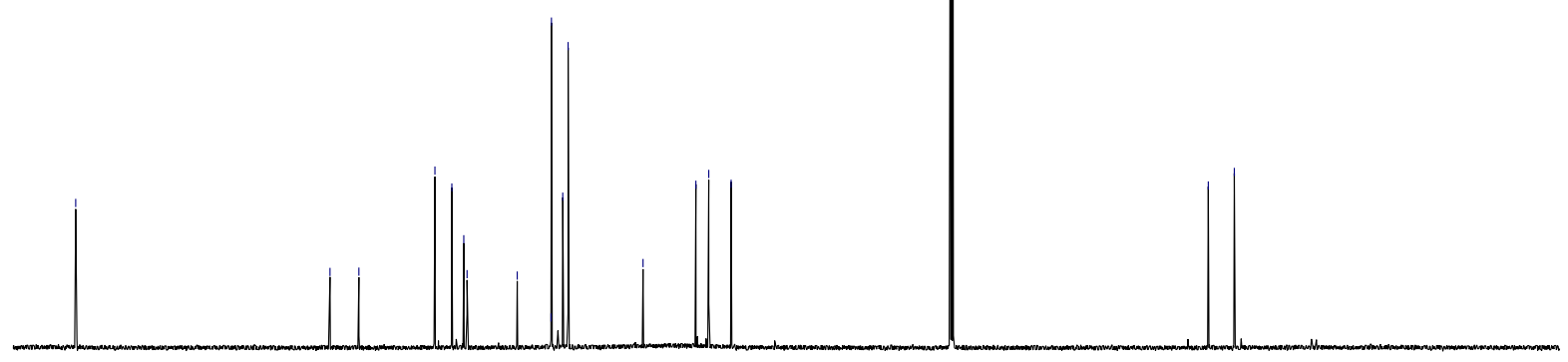

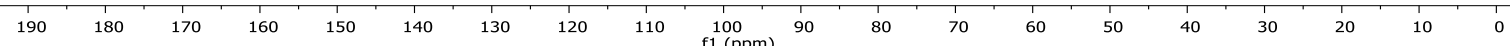


(6R,7S)-6-(Furan-2-yl)-7-phenyl-6,7-dihydrobenzofuran-5-carbaldehyde 3i

${ }^{1} \mathrm{H}$ NMR (700 MHz, $\left.\mathrm{CDCl}_{3}\right)$

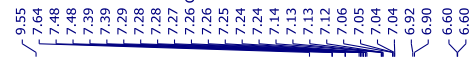

(1)

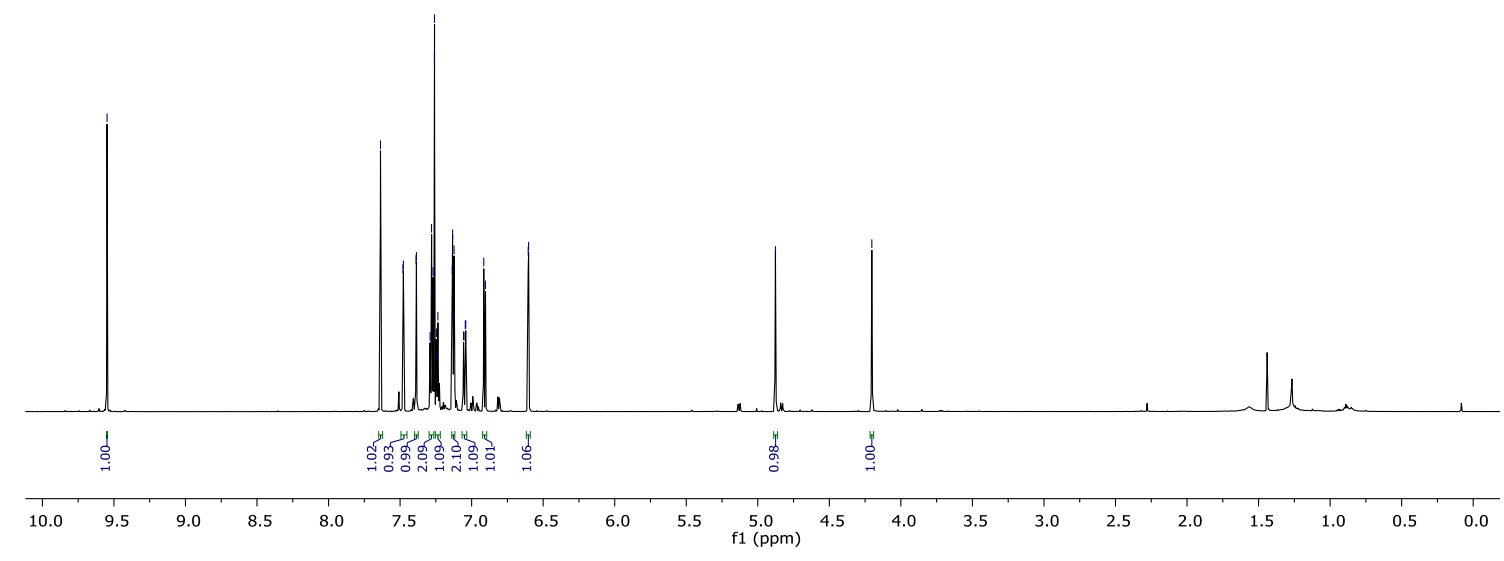

${ }^{13} \mathrm{C}$ NMR (176 $\left.\mathrm{MHz}, \mathrm{CDCl}_{3}\right)$

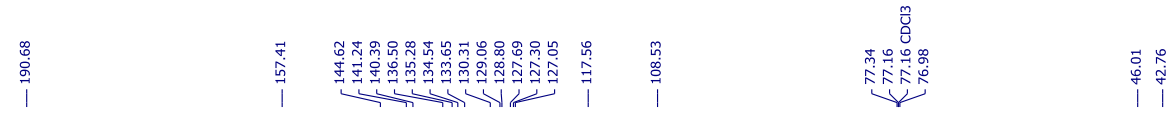

(1)
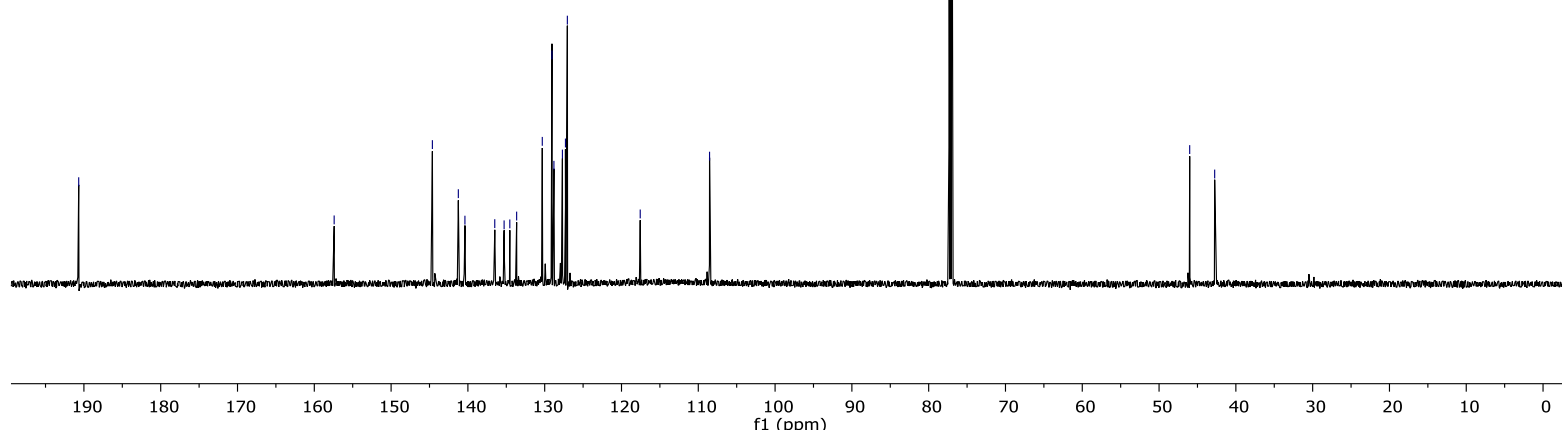
(6S,7S)-7-(4-Fluorophenyl)-6-phenyl-6,7-dihydrobenzofuran-5-carbaldehyde 3j

${ }^{1} \mathrm{H}$ NMR (700 MHz, $\left.\mathrm{CDCl}_{3}\right)$

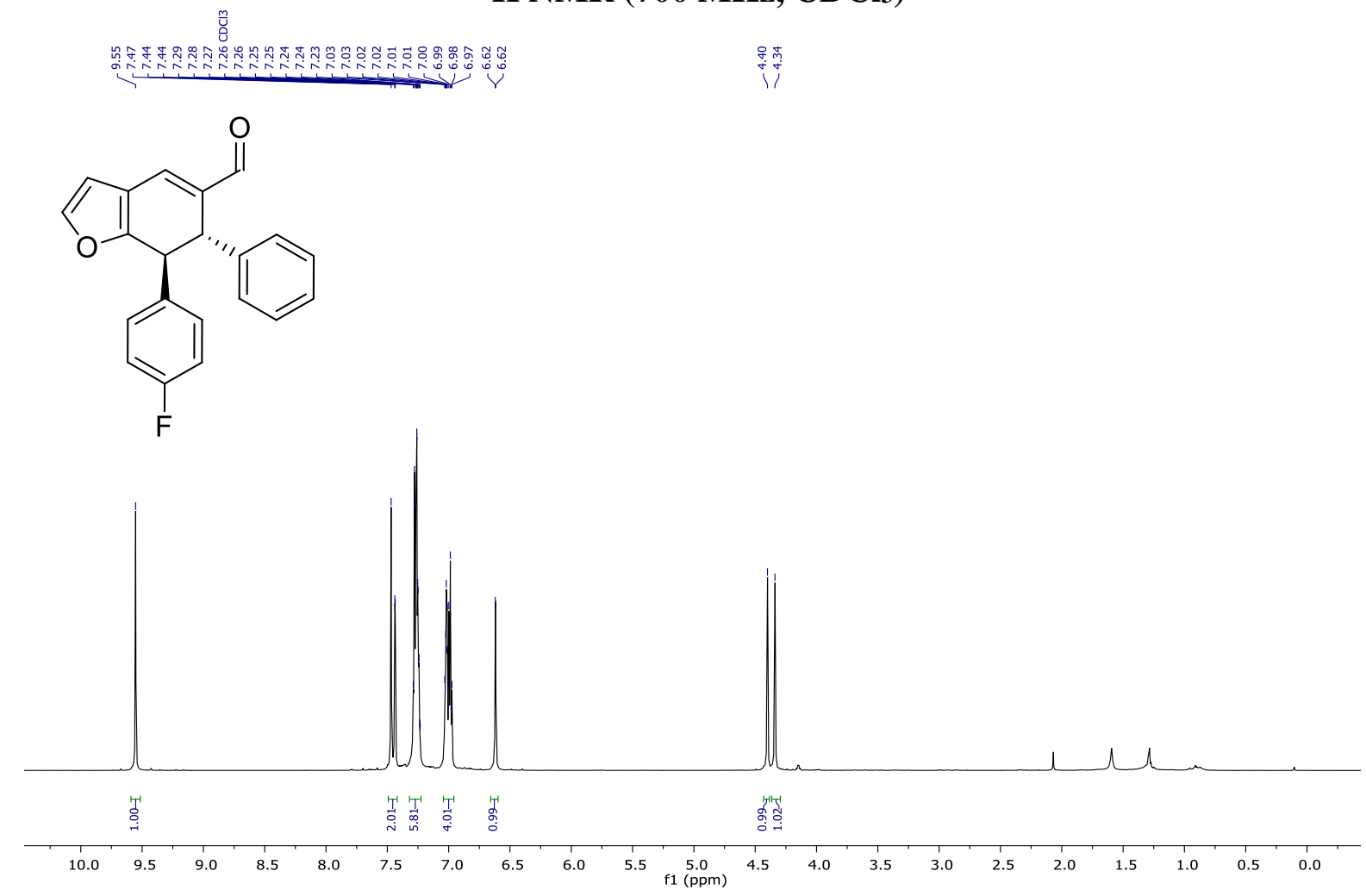

${ }^{13} \mathrm{C}$ NMR (176 MHz, $\left.\mathrm{CDCl}_{3}\right)$
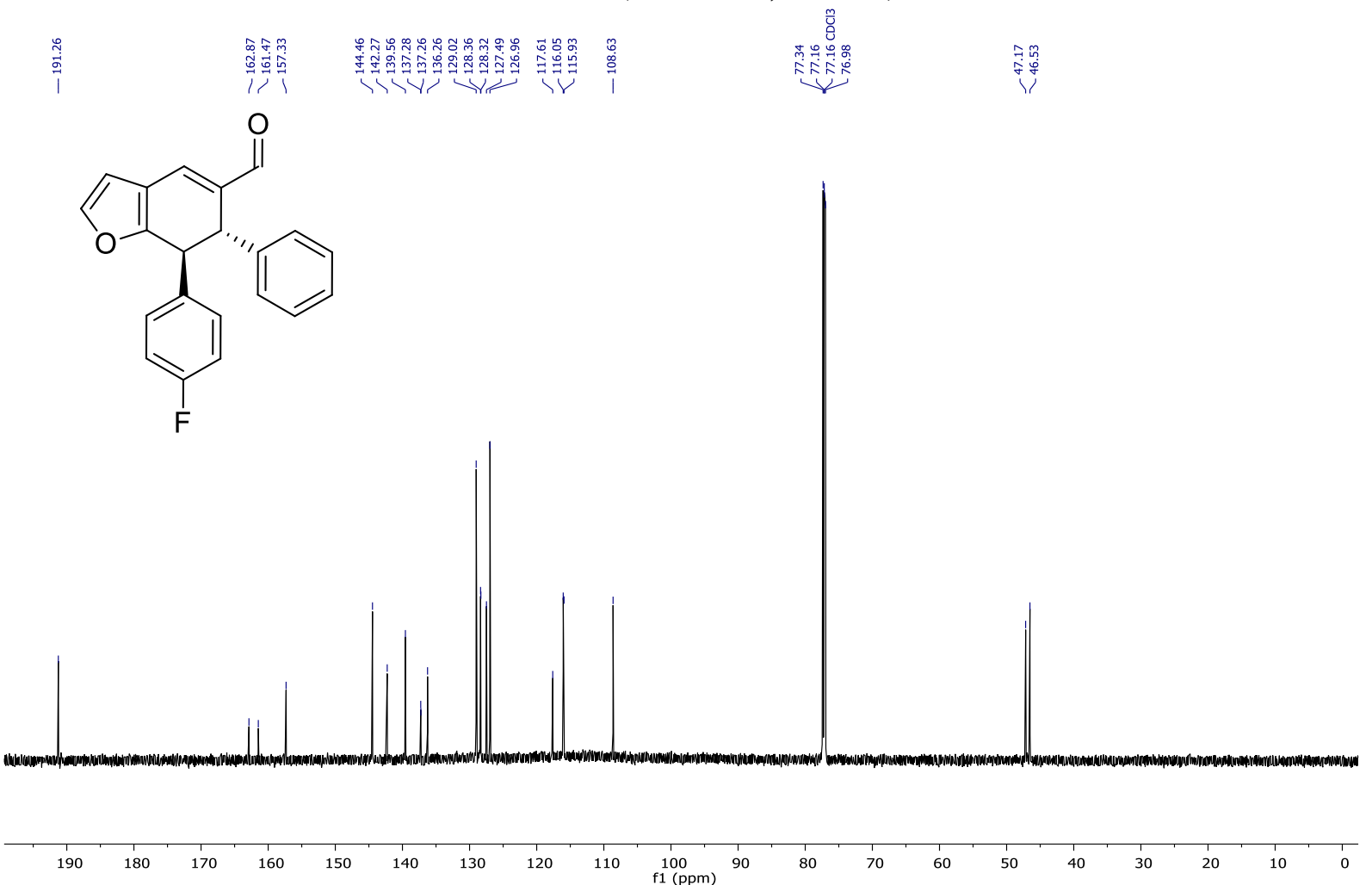
(6S,7S)-6-Phenyl-7-(p-tolyl)-6,7-dihydrobenzofuran-5-carbaldehyde 3k

${ }^{1} \mathrm{H}$ NMR (700 MHz, $\left.\mathrm{CDCl}_{3}\right)$
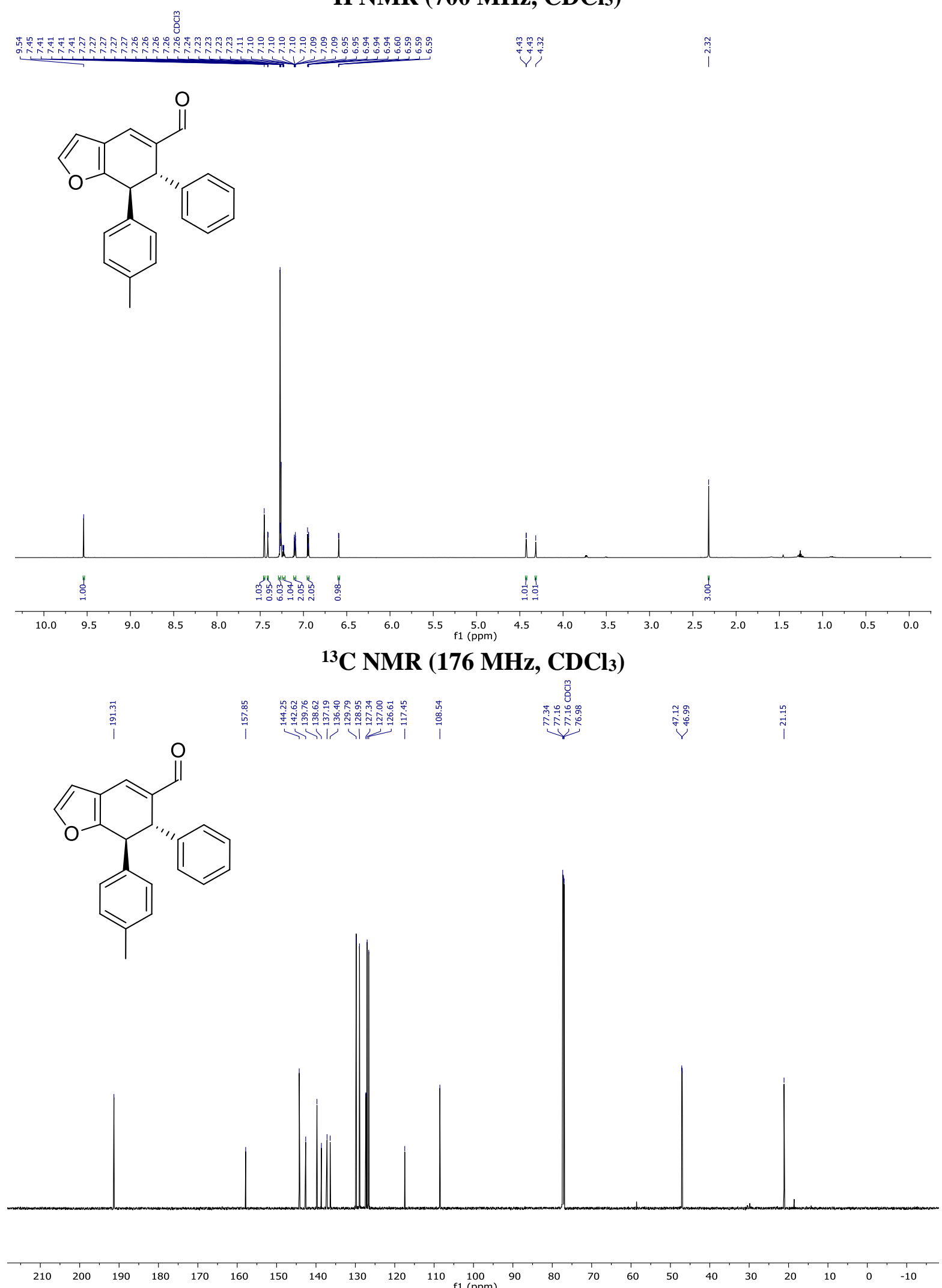
(6S,7S)-7-(3-Methoxyphenyl)-6-phenyl-6,7-dihydrobenzofuran-5-carbaldehyde 31

${ }^{1} \mathrm{H}$ NMR (700 MHz, $\left.\mathrm{CDCl}_{3}\right)$

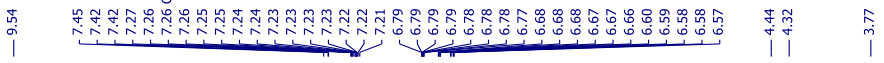<smiles>COc1cccc([C@H]2c3occc3C=C(C=O)[C@H]2c2ccccc2)c1</smiles>

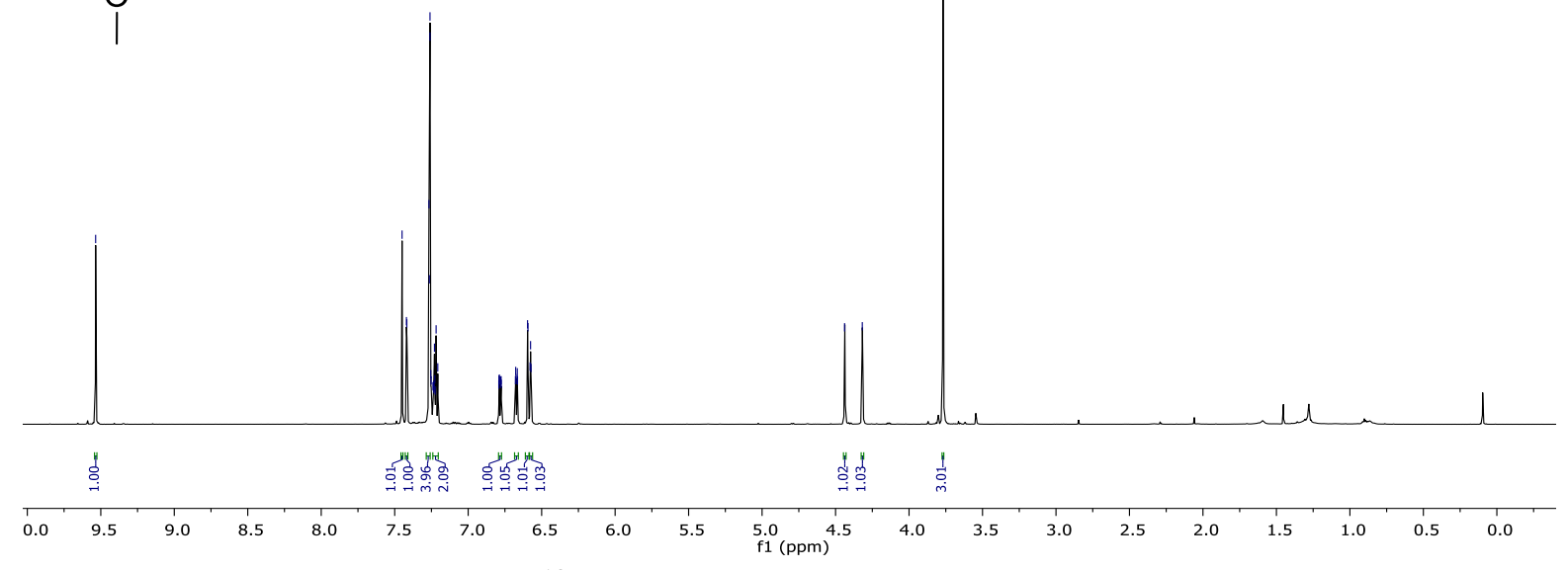

${ }^{13} \mathrm{C}$ NMR (176 MHz, $\left.\mathrm{CDCl} 3\right)$
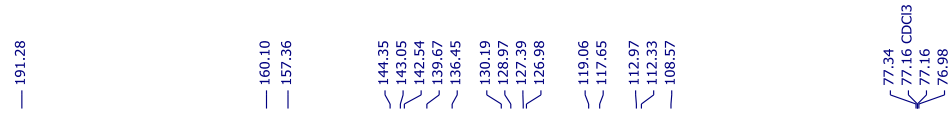

i<smiles>COc1cccc([C@H]2c3occc3C=C(C=O)[C@H]2c2ccccc2)c1</smiles>

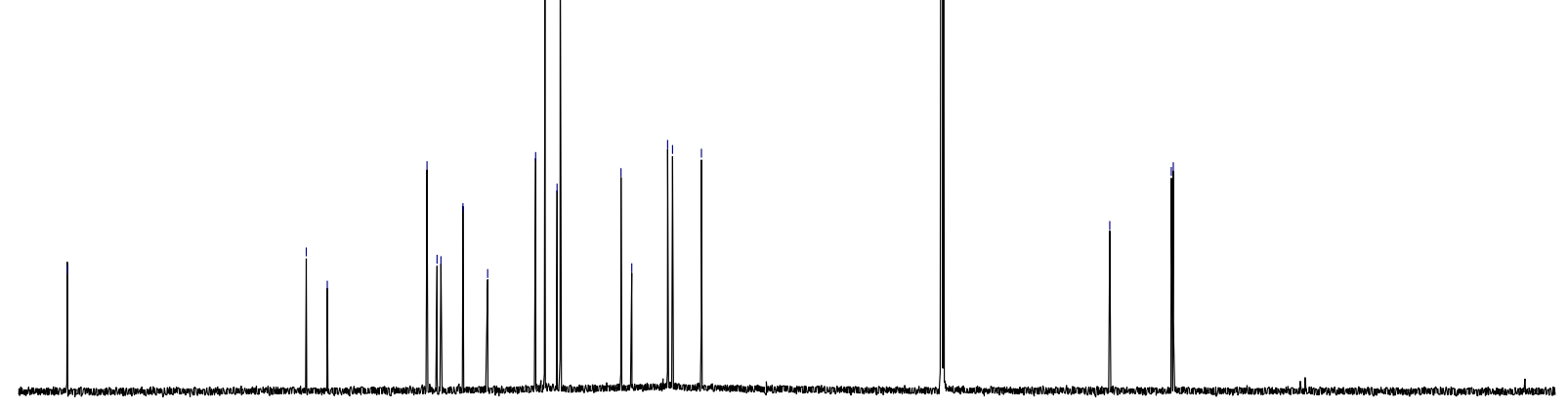

190

$150 \quad 140$

10090 
(6S,7S)-6-Phenyl-7-(o-tolyl)-6,7-dihydrobenzofuran-5-carbaldehyde 3m ${ }^{1} \mathrm{H}$ NMR (700 MHz, $\left.\mathrm{CDCl}_{3}\right)$

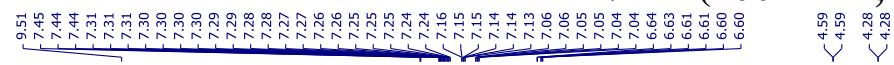

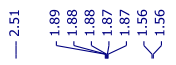<smiles>Cc1ccccc1C1c2occc2C=C(C=O)C1c1ccccc1</smiles>

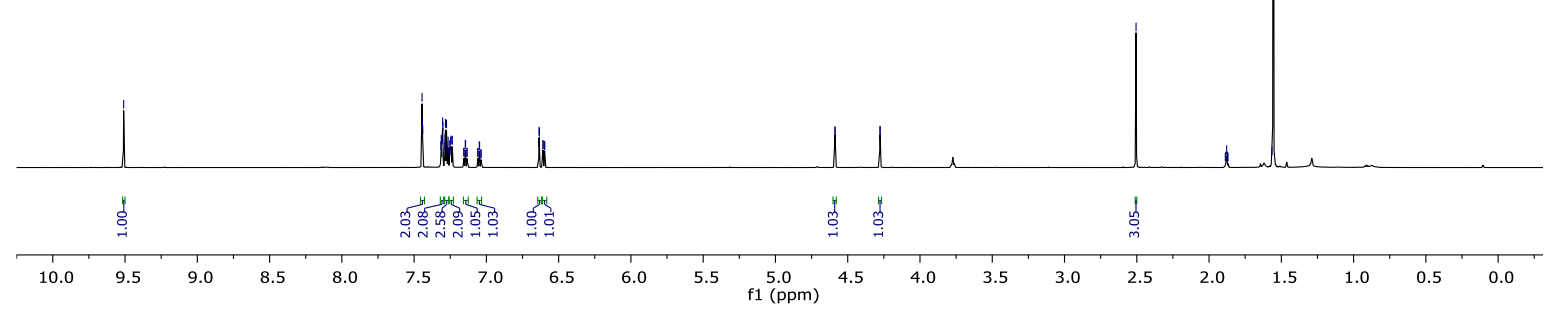

${ }^{13} \mathrm{C}$ NMR (176 MHz, $\left.\mathrm{CDCl}_{3}\right)$
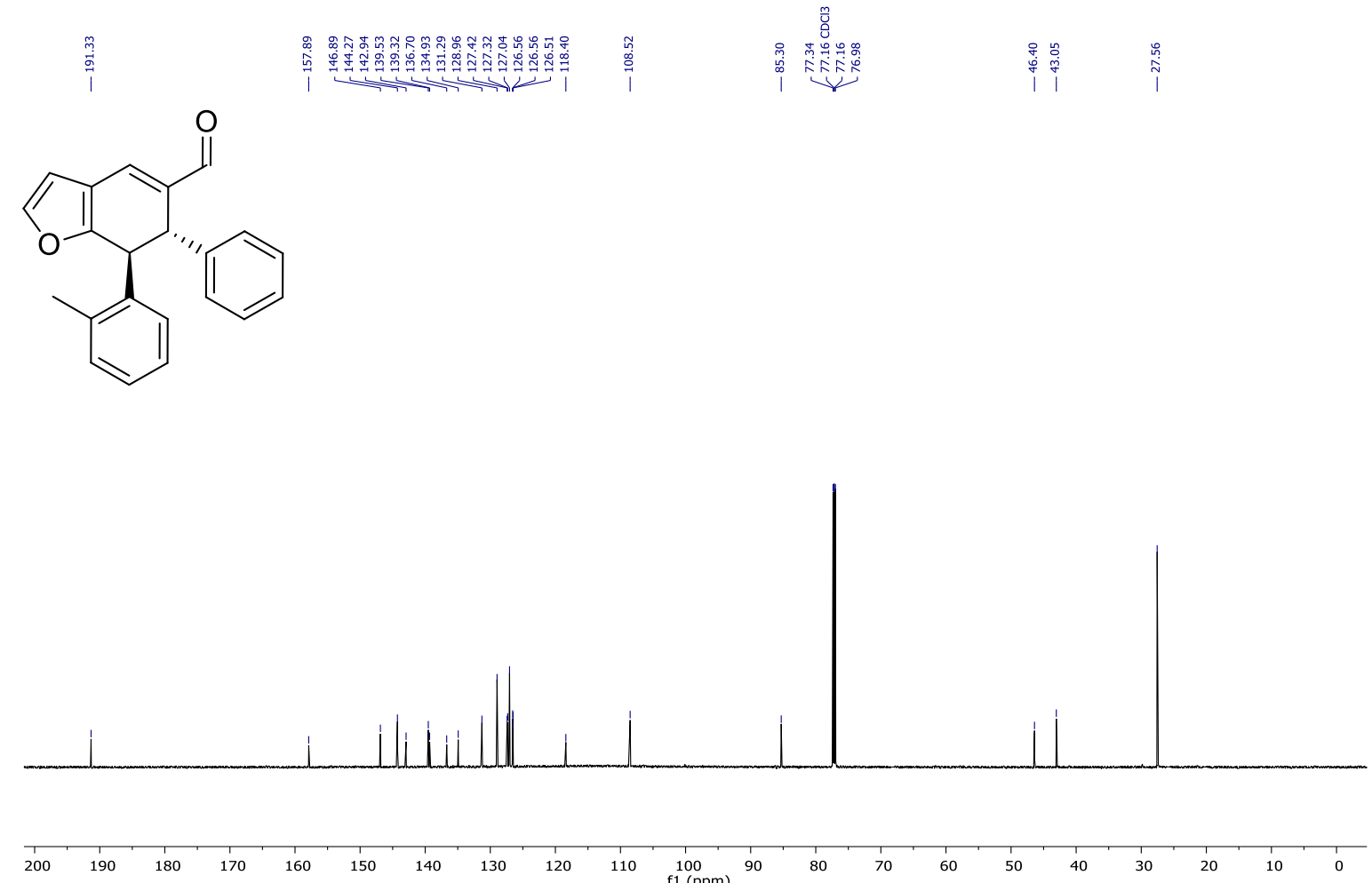
(6S,7R)-6-Phenyl-7-vinyl-6,7-dihydrobenzofuran-5-carbaldehyde 3n

${ }^{1} \mathrm{H}$ NMR (700 $\left.\mathrm{MHz}, \mathrm{CDCl}_{3}\right)$

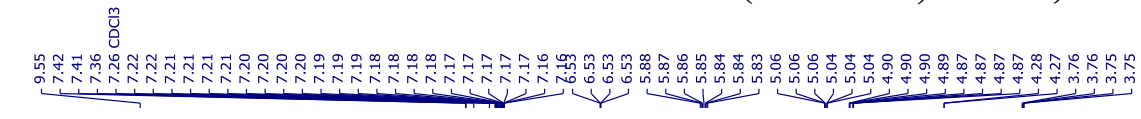<smiles>C=C[C@H]1c2occc2C=C(C=O)[C@H]1c1ccccc1</smiles>

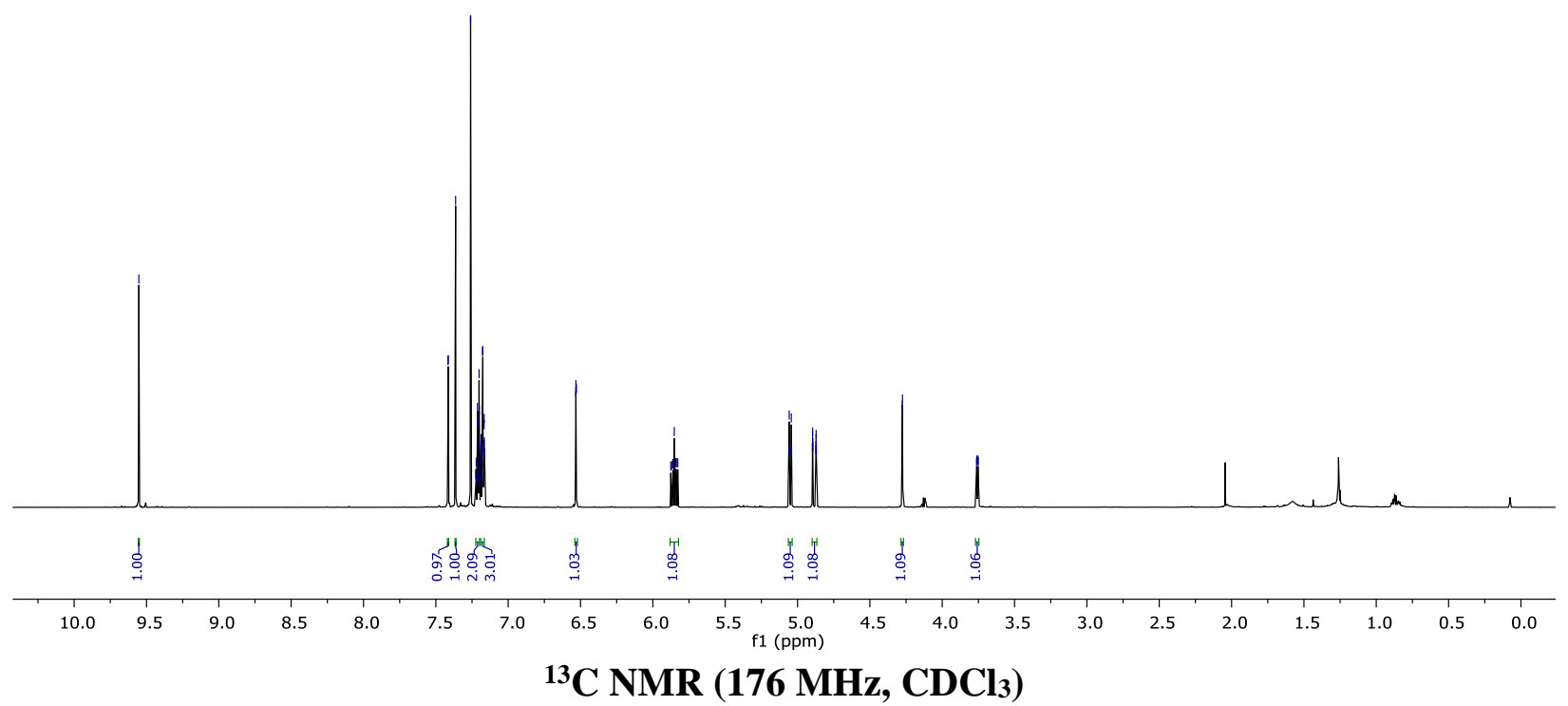

$$
\text { ज़े }
$$
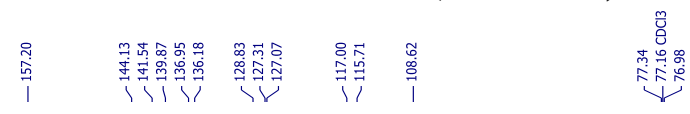<smiles>C=C[C@H]1c2occc2C=C(C=O)[C@H]1c1ccccc1</smiles>

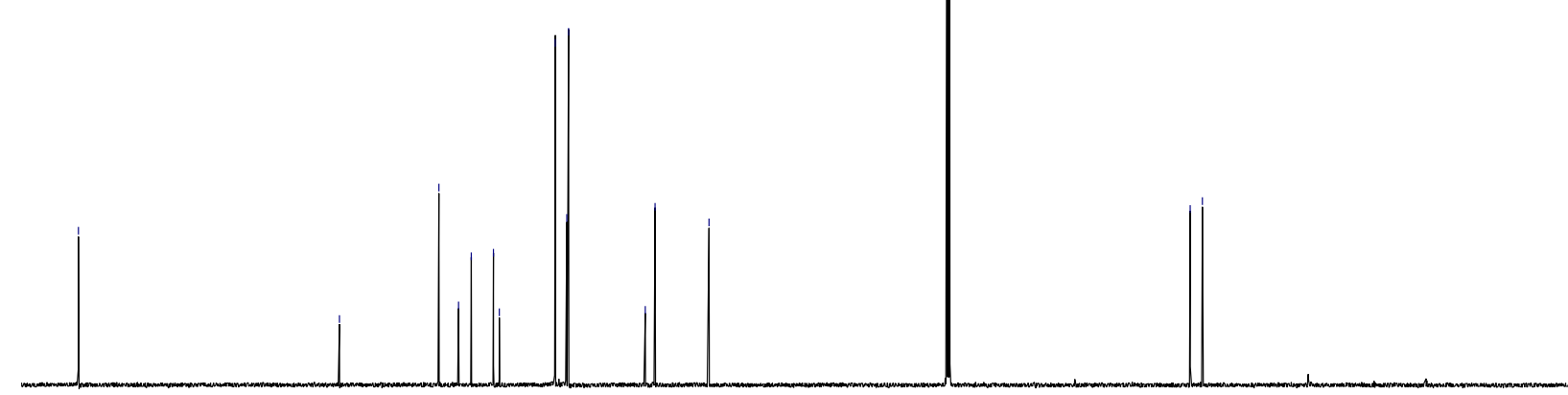

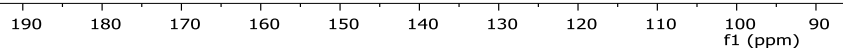

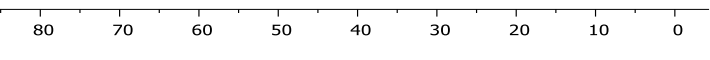


(3S,4S)-3,4-Diphenyl-3,4-dihydrodibenzo[b,d]furan-2-carbaldehyde 30 ${ }^{1} \mathrm{H}$ NMR (700 MHz, $\left.\mathrm{CDCl}_{3}\right)$

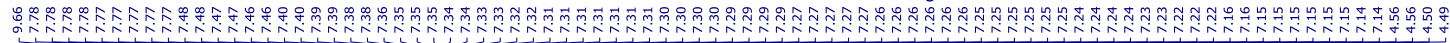<smiles>O=CC1=Cc2c(oc3ccccc23)C(c2ccccc2)[C@@H]1c1ccccc1</smiles>
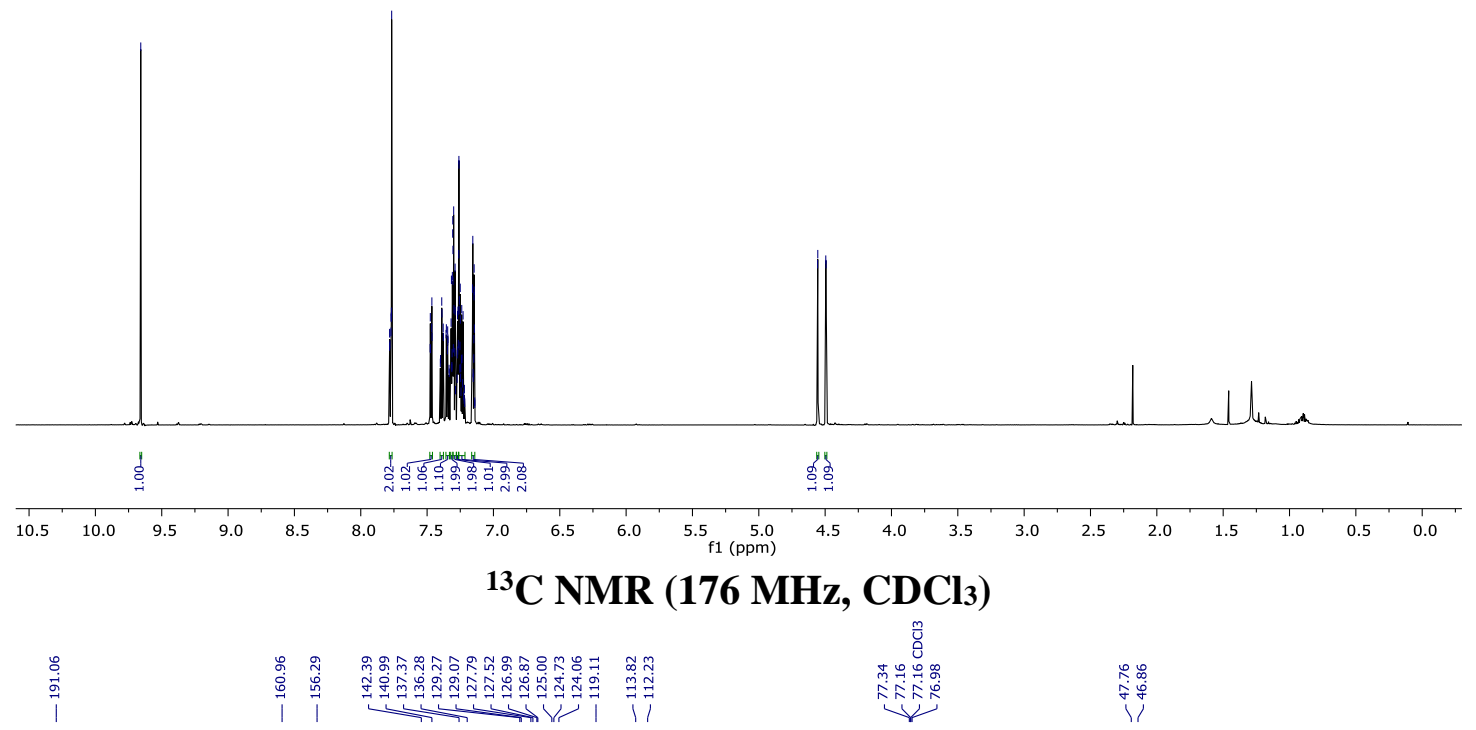

ไ゚.<smiles>O=CC1=Cc2c(oc3ccccc23)[C@@H](c2ccccc2)[C@H]1c1ccccc1</smiles>
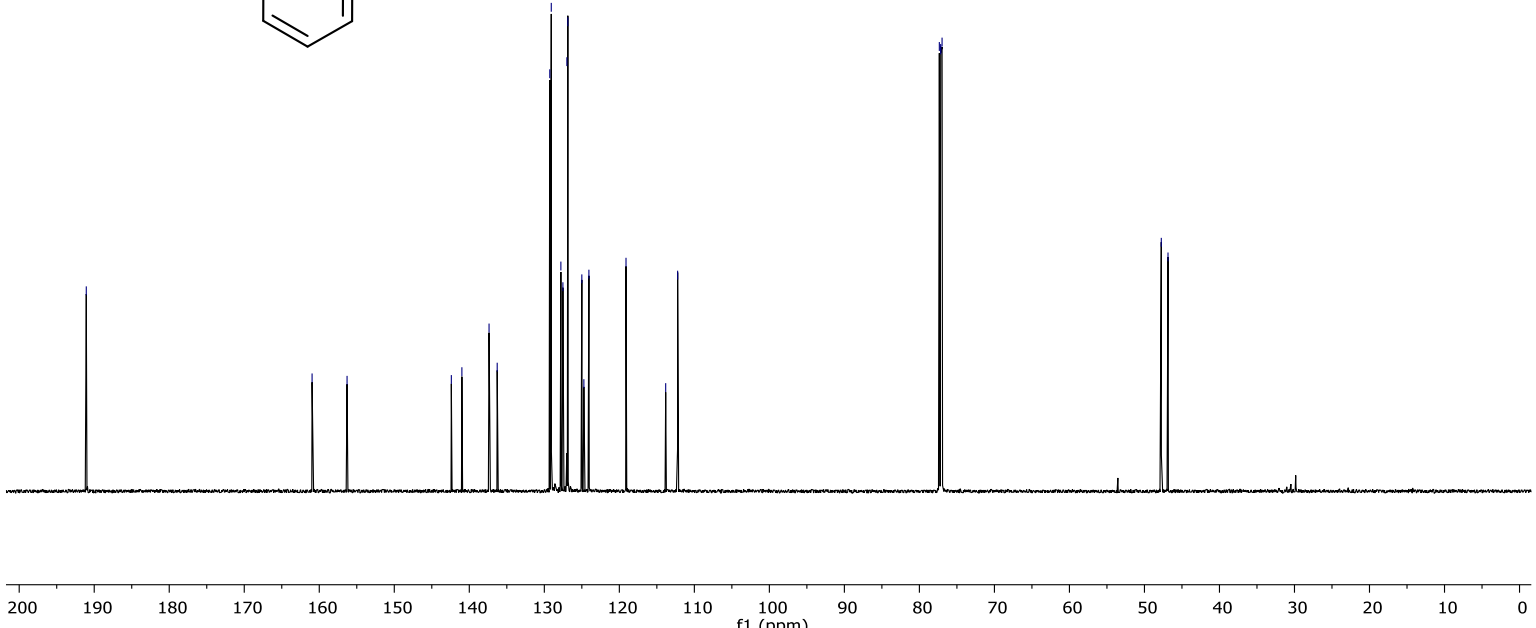


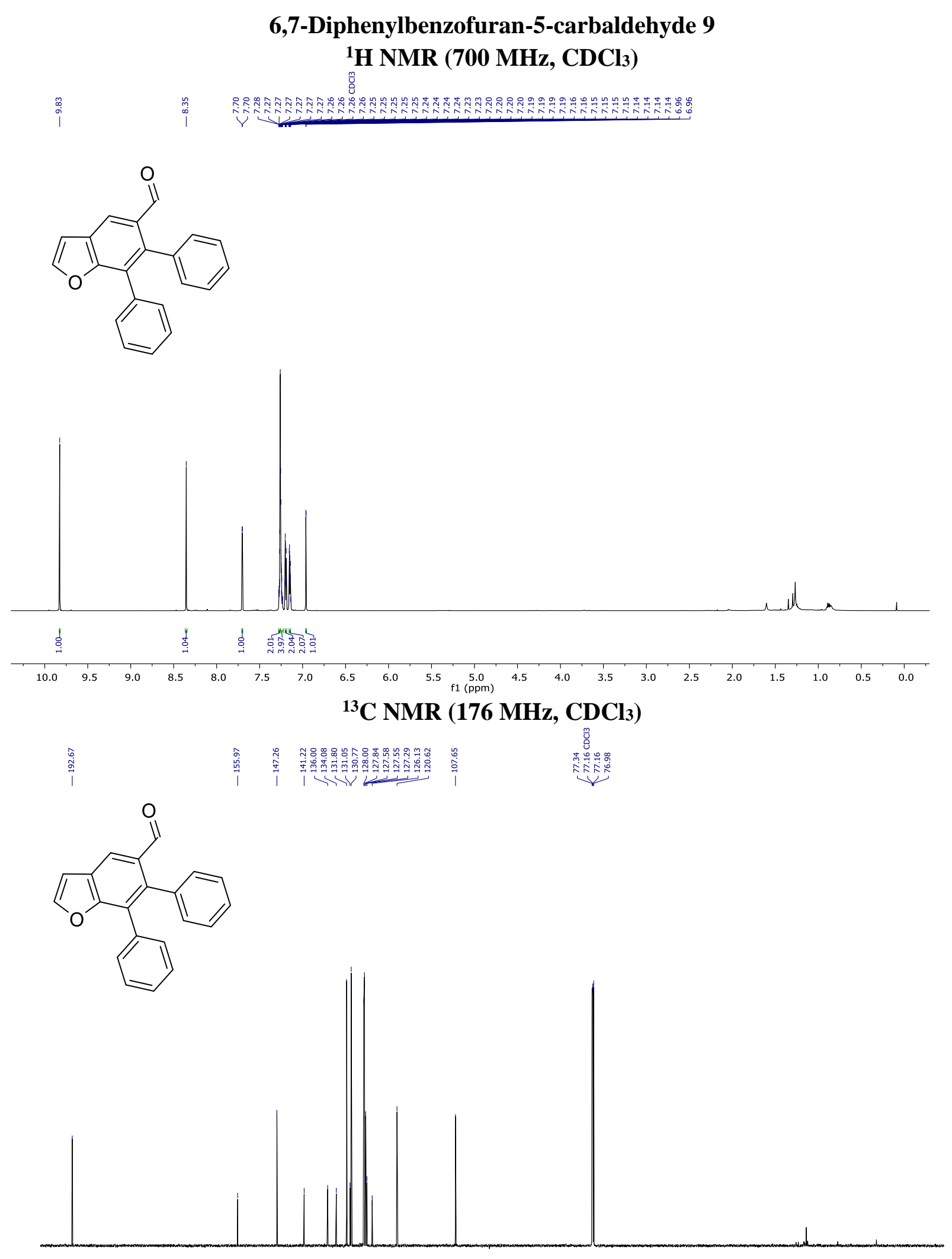

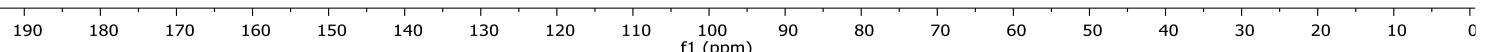



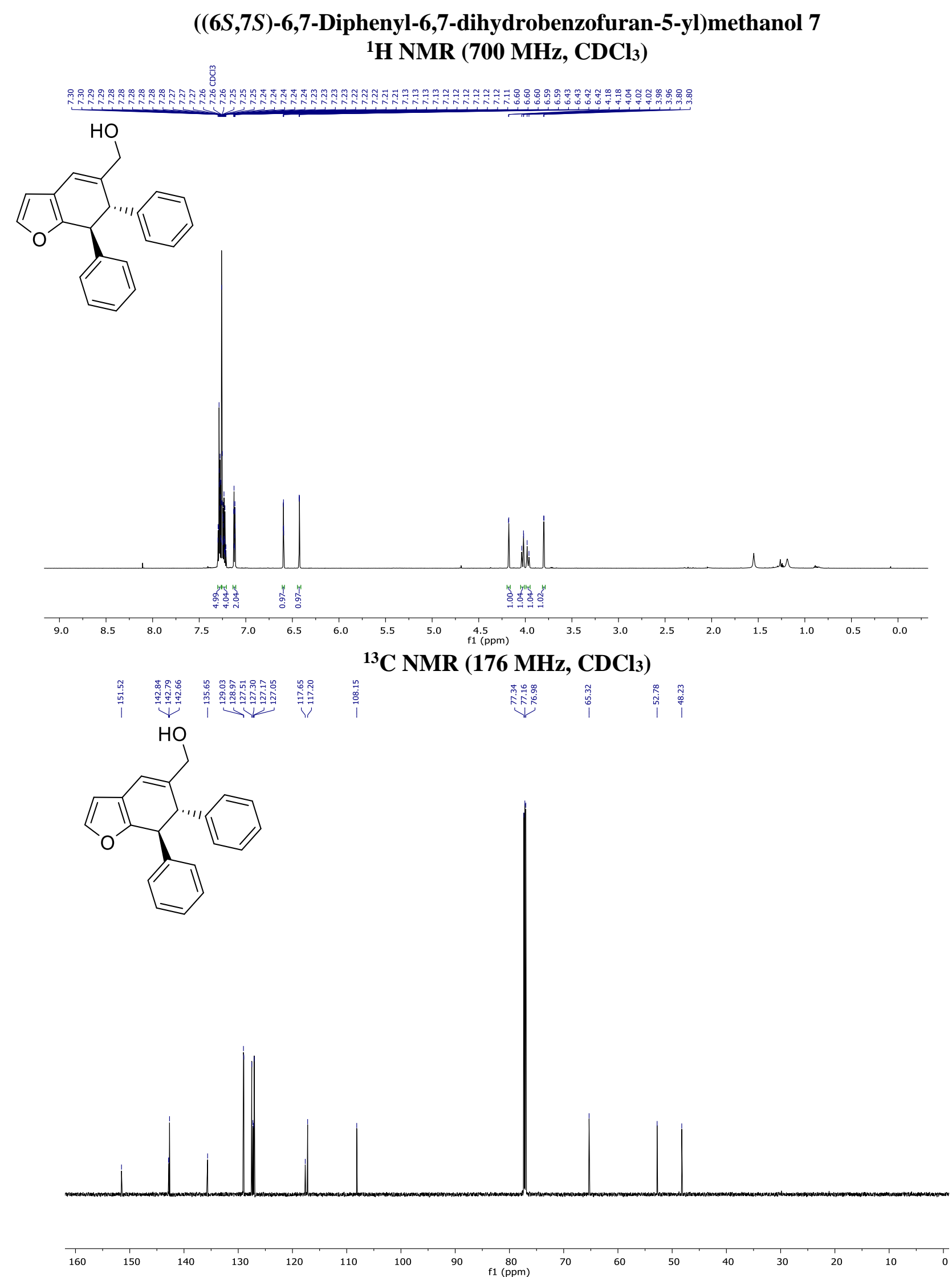
$(4 S, 5 S)-4,5$-Diphenyl-5,12-dihydro-4H-benzo[b]benzofuro[4,5-e][1,4]diazepine 8 .

${ }^{1} \mathrm{H}$ NMR (700 MHz, $\left.\mathrm{CDCl}_{3}\right)$

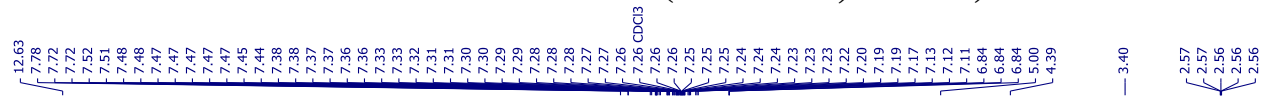<smiles>C1=Nc2ccccc2NC2=C1[C@H](c1ccccc1)[C@@H](c1ccccc1)[C@@H](c1ccccc1)c1occc12</smiles>

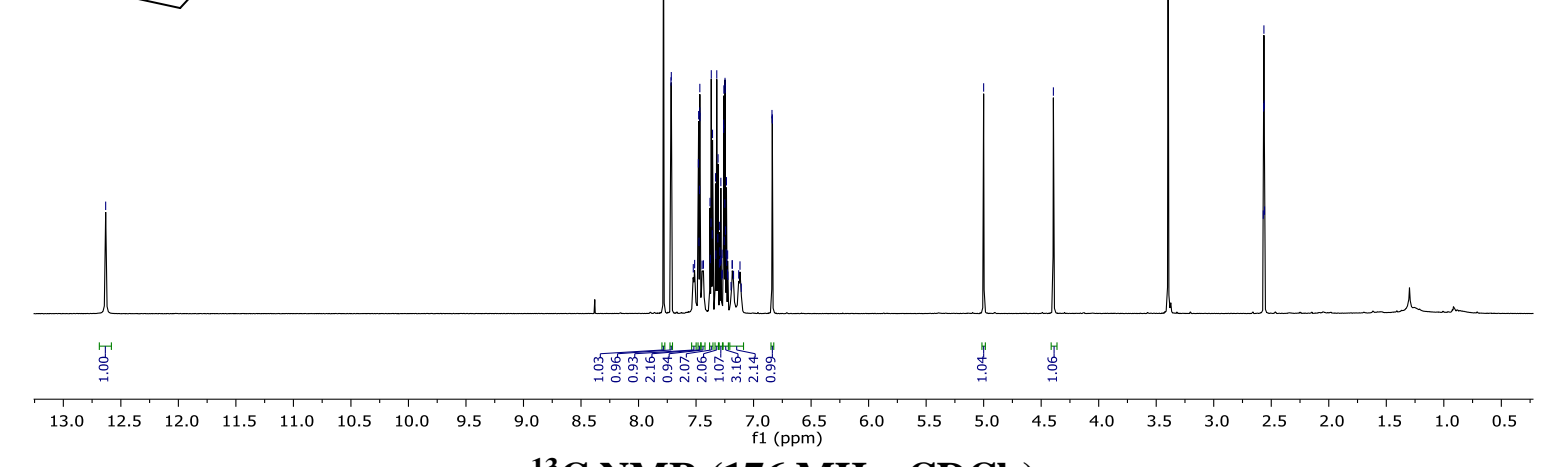

${ }^{13} \mathrm{C}$ NMR (176 MHz, $\left.\mathrm{CDCl}_{3}\right)$
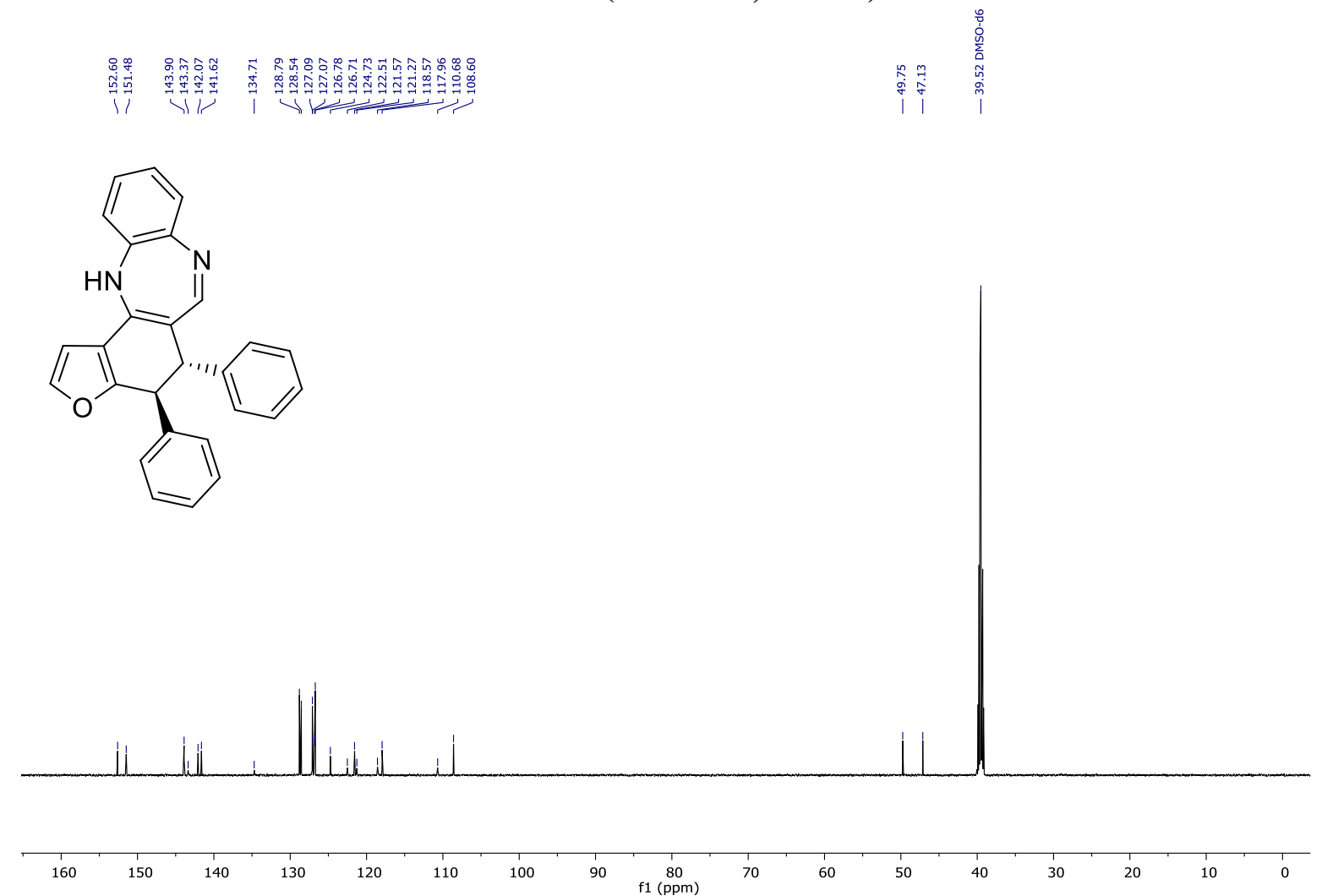
7. $\mathrm{UPC}^{2}$ Data

(6S,7S)-6,7-Diphenyl-6,7-dihydrobenzofuran-5-carbaldehyde 3a

Racemic sample

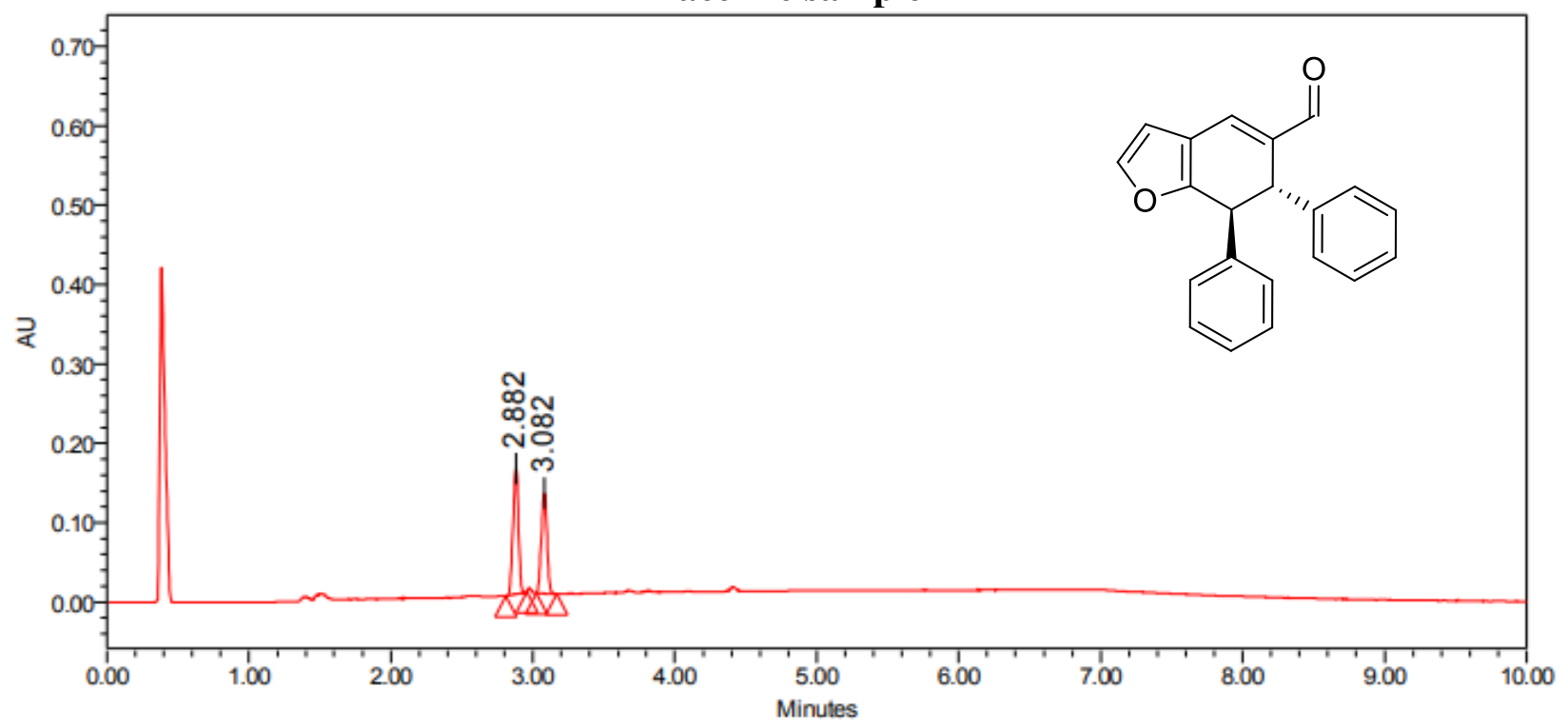

Peak Results

\begin{tabular}{|l|c|r|}
\hline & RT & \% Area \\
\hline 1 & 2.882 & 54.93 \\
\hline 2 & 3.082 & 45.07 \\
\hline
\end{tabular}

Enantiomerically enriched sample

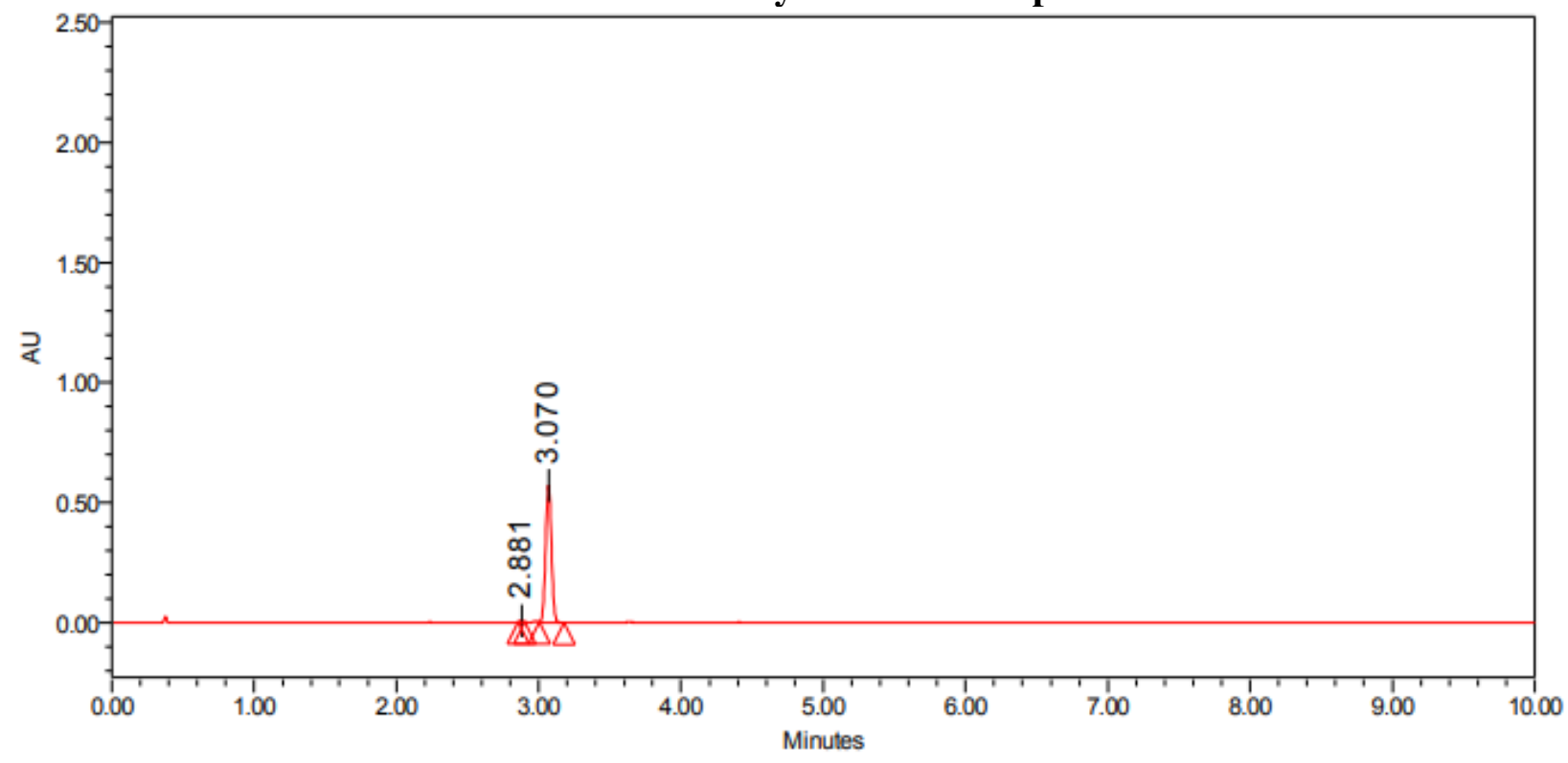

Peak Results

\begin{tabular}{|c|c|r|}
\hline & RT & \% Area \\
\hline 1 & 2.881 & 0.44 \\
\hline 2 & 3.070 & 99.56 \\
\hline
\end{tabular}


(6S,7S)-6-(4-Nitrophenyl)-7-phenyl-6,7-dihydrobenzofuran-5-carbaldehyde 3b

Racemic sample

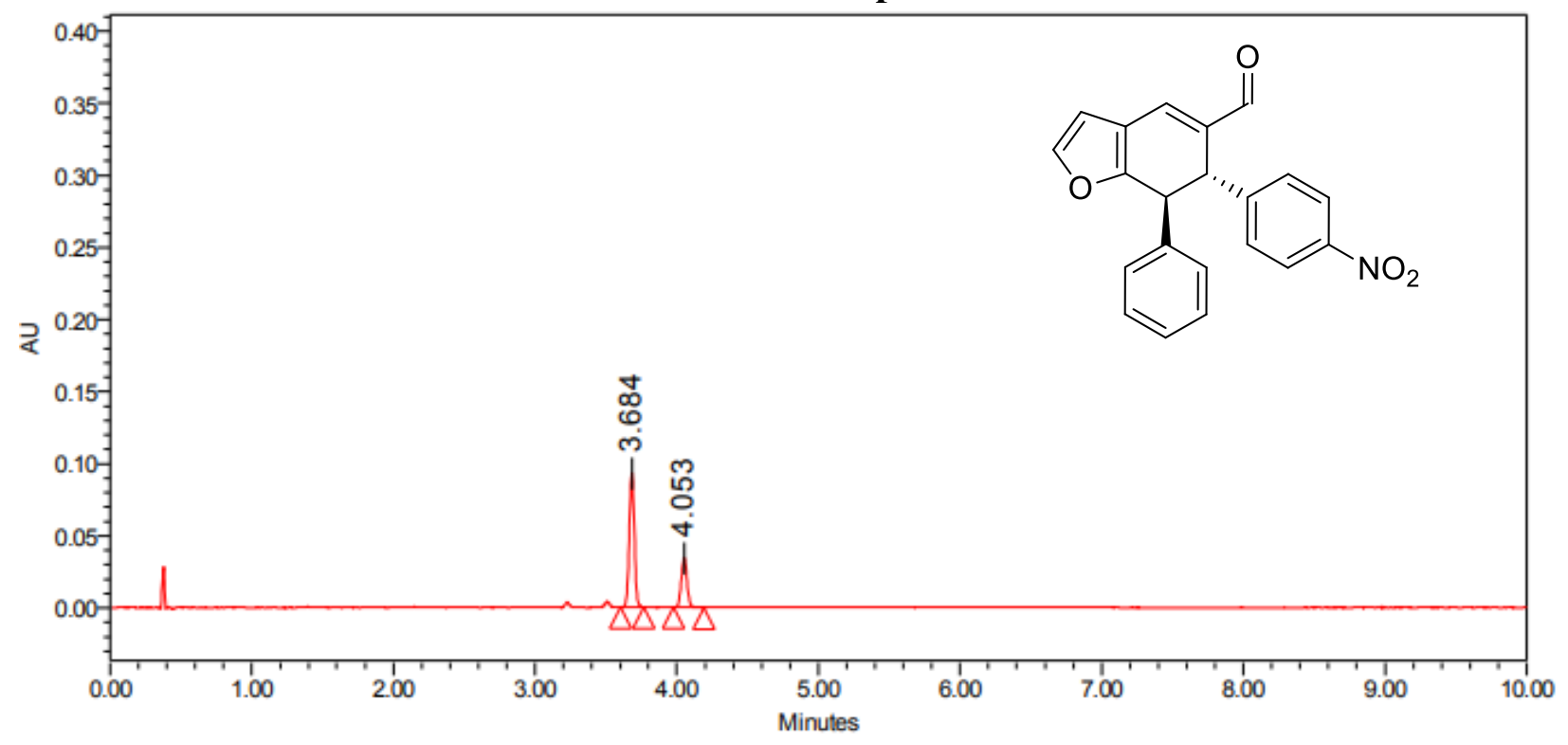

Peak Results

\begin{tabular}{|l|c|r|}
\hline & RT & \% Area \\
\hline 1 & 3.684 & 70.99 \\
\hline 2 & 4.053 & 29.01 \\
\hline
\end{tabular}

Enantiomerically enriched sample

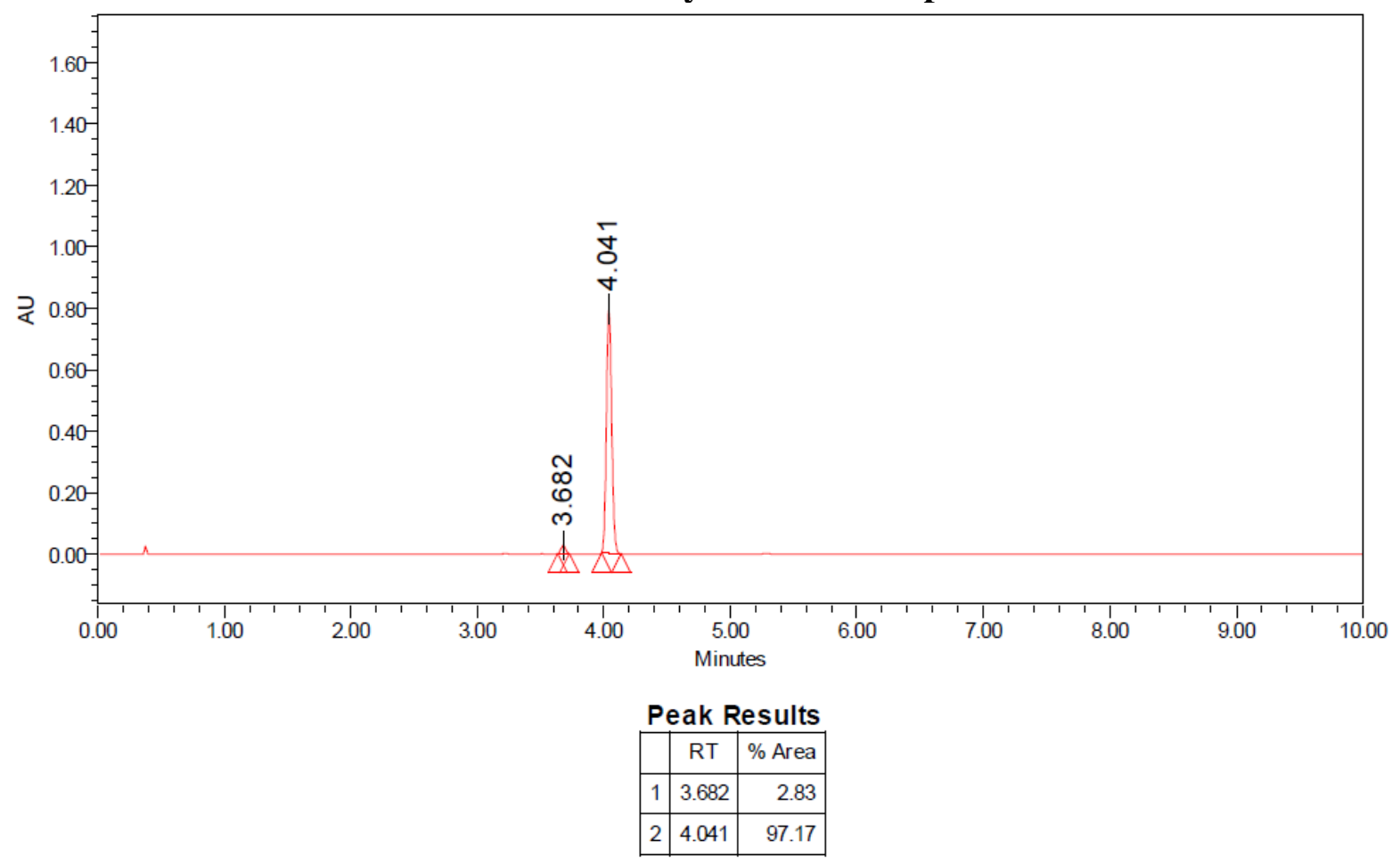


(6S,7S)-6-(4-Chlorophenyl)-7-phenyl-6,7-dihydrobenzofuran-5-carbaldehyde 3c
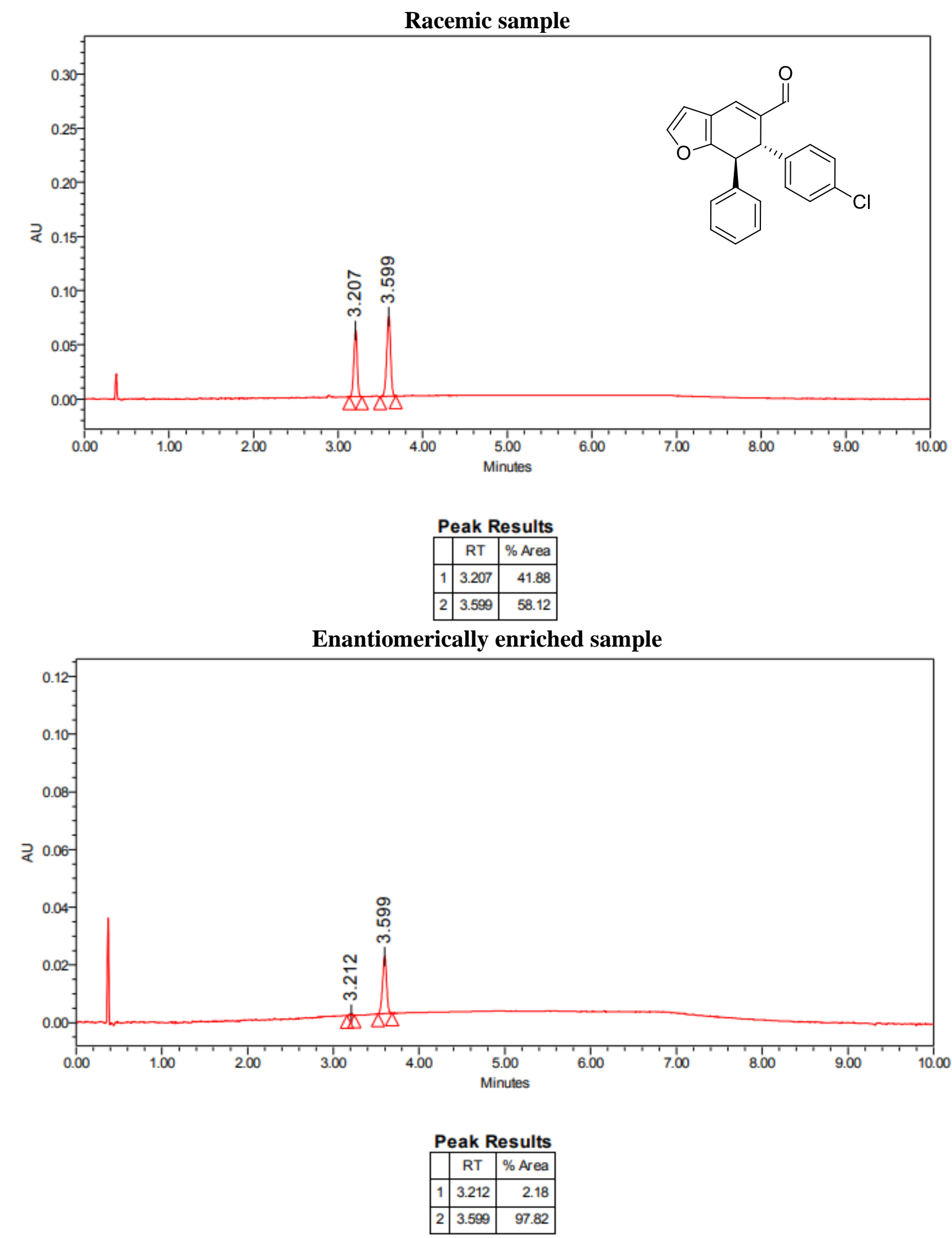
(6S,7S)-7-Phenyl-6-(p-tolyl)-6,7-dihydrobenzofuran-5-carbaldehyde 3d

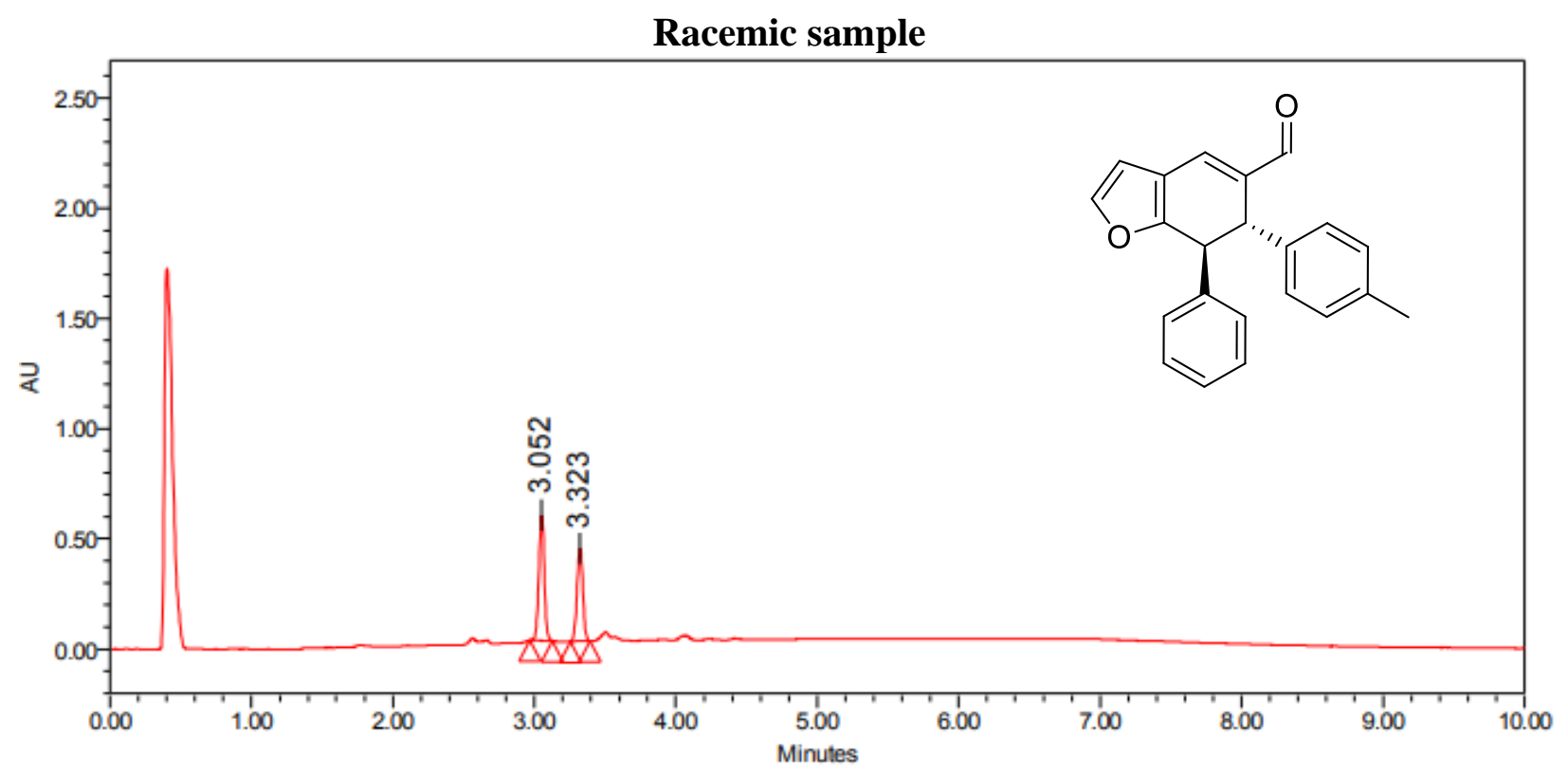

Peak Results

\begin{tabular}{|c|c|r|}
\hline & RT & \% Area \\
\hline 1 & 3.052 & 55.83 \\
\hline 2 & 3.323 & 44.17 \\
\hline
\end{tabular}

Enantiomerically enriched sample

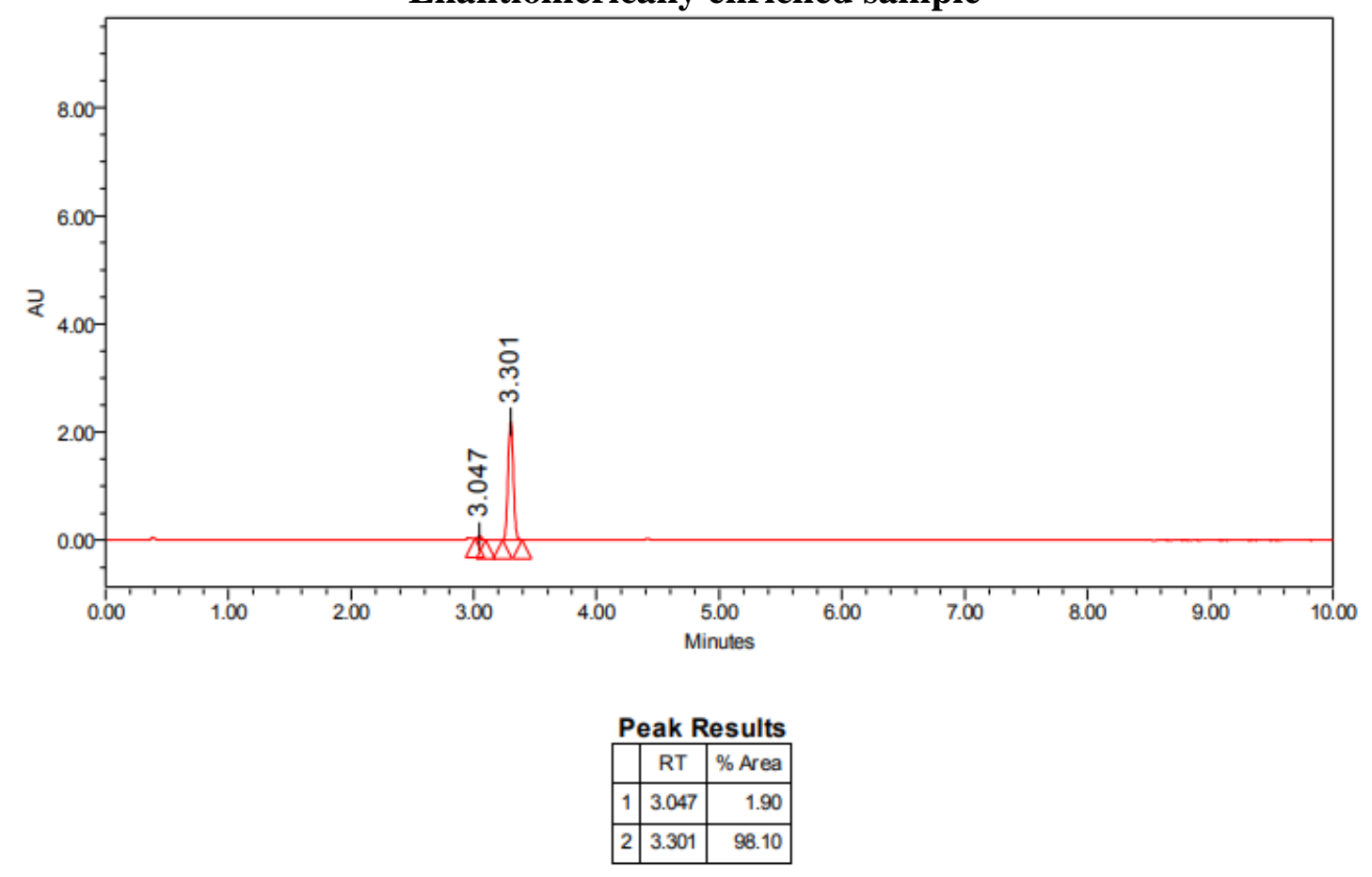


(6S,7S)-6-(4-Methoxyphenyl)-7-phenyl-6,7-dihydrobenzofuran-5-carbaldehyde 3e

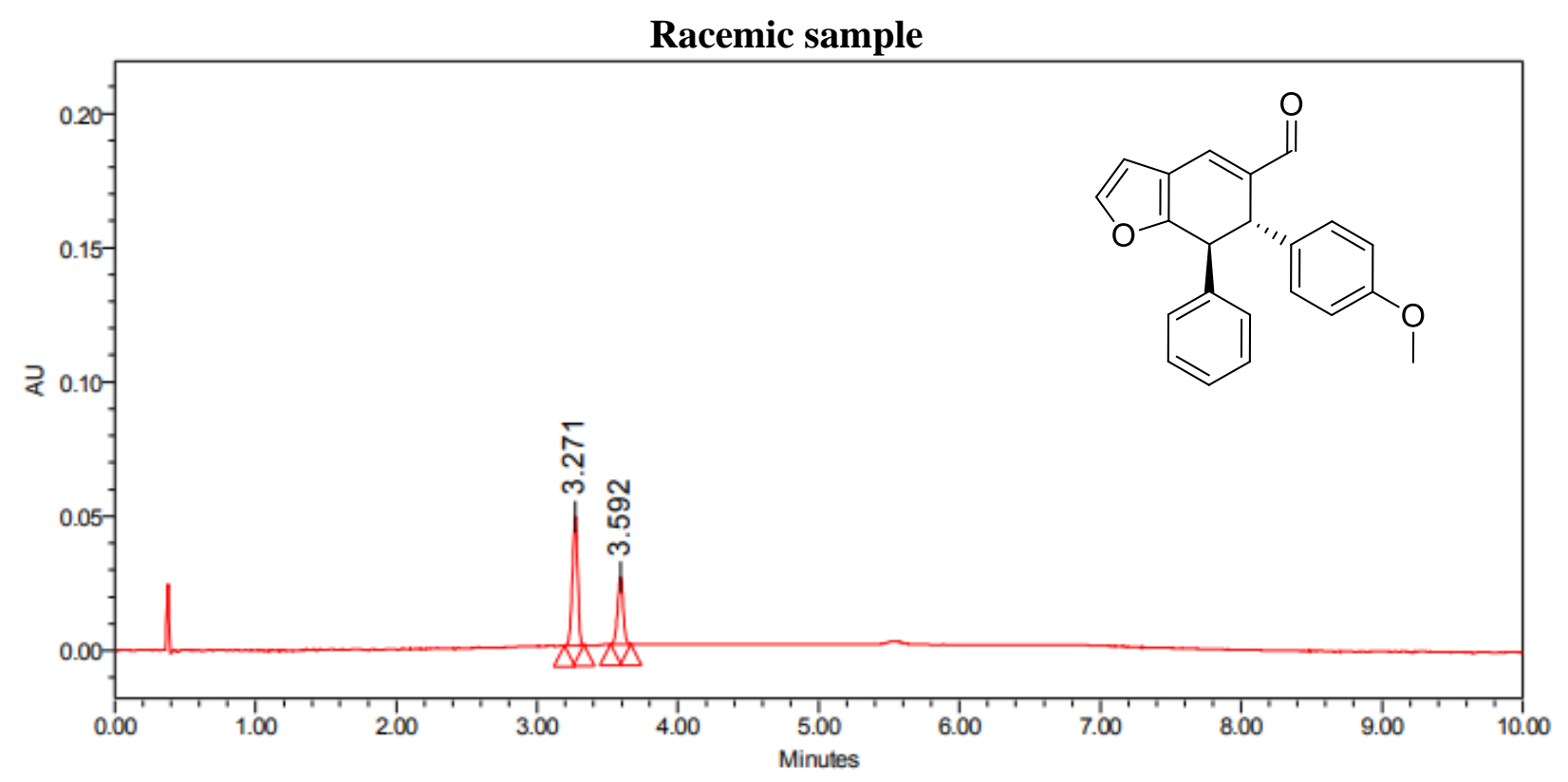

Peak Results
\begin{tabular}{|c|c|r|}
\hline & RT & $\%$ Area \\
\hline 1 & 3.271 & 64.19 \\
\hline 2 & 3.592 & 35.81 \\
\hline
\end{tabular}

Enantiomerically enriched sample

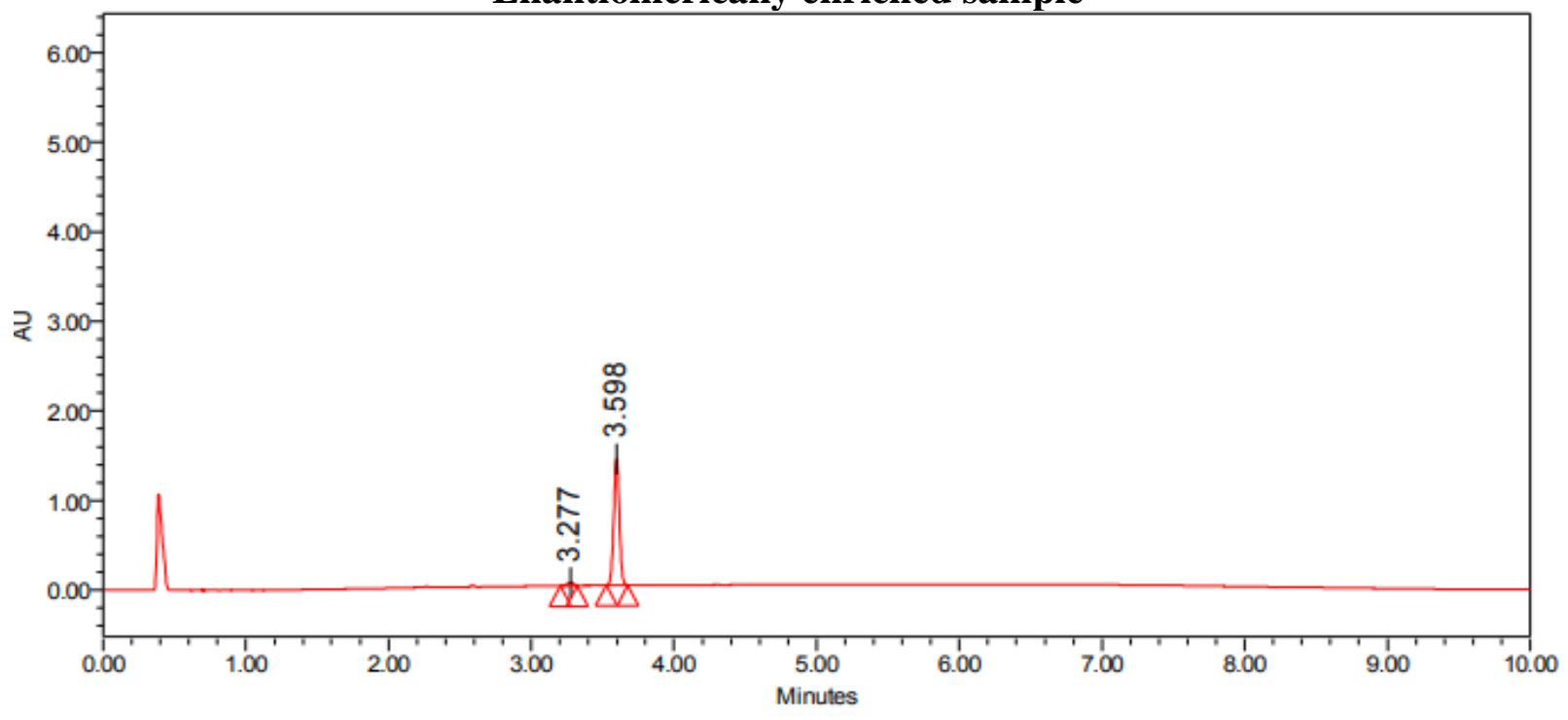

Peak Results

\begin{tabular}{|c|c|r|}
\hline & RT & \% Area \\
\hline 1 & 3.277 & 2.33 \\
\hline 2 & 3.598 & 97.67 \\
\hline
\end{tabular}


(6S,7S)-6-(3-Methoxyphenyl)-7-phenyl-6,7-dihydrobenzofuran-5-carbaldehyde 3f

Racemic sample

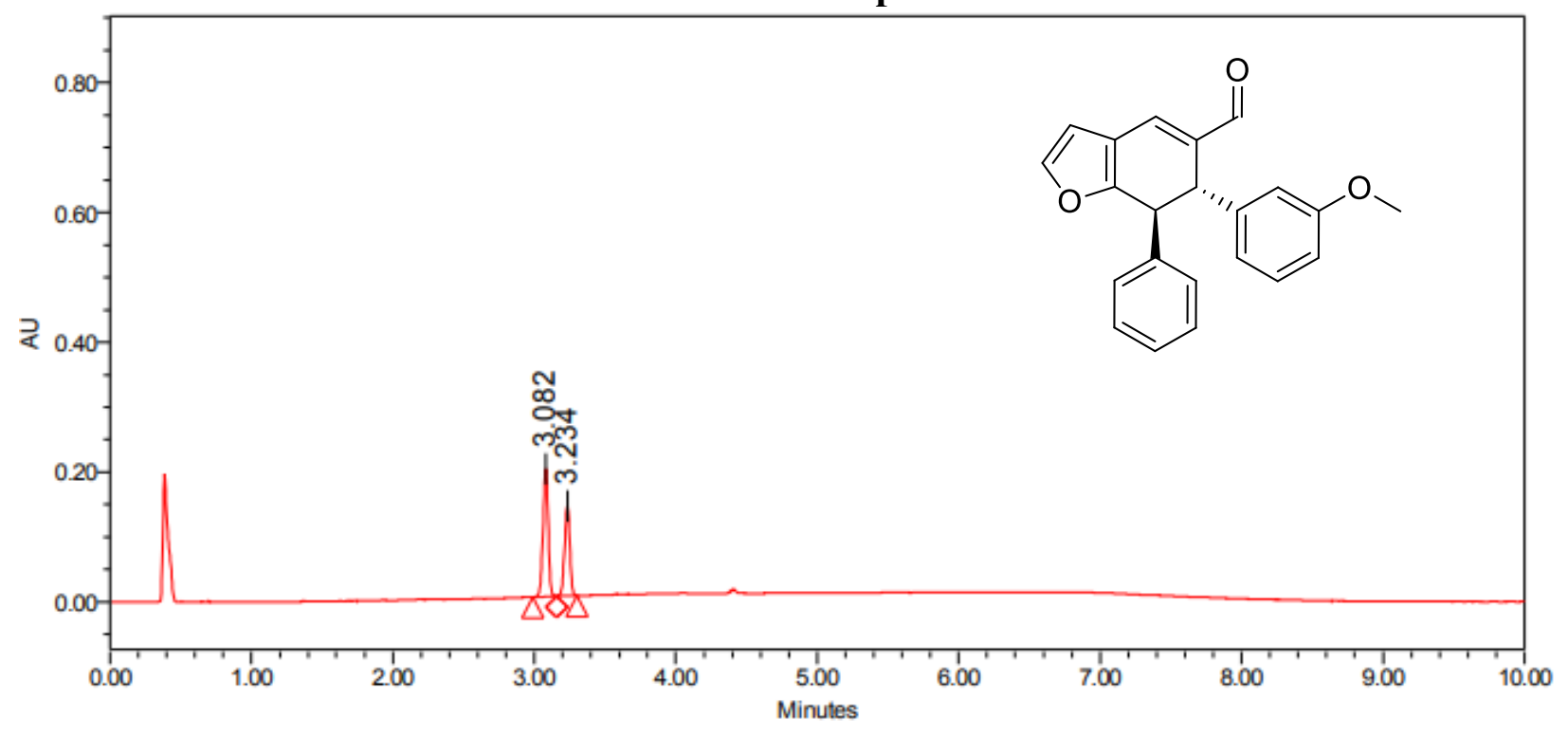

Peak Results

\begin{tabular}{|l|c|r|}
\hline & RT & \% Area \\
\hline 1 & 3.082 & 58.55 \\
\hline 2 & 3.234 & 41.45 \\
\hline
\end{tabular}

Enantiomerically enriched sample

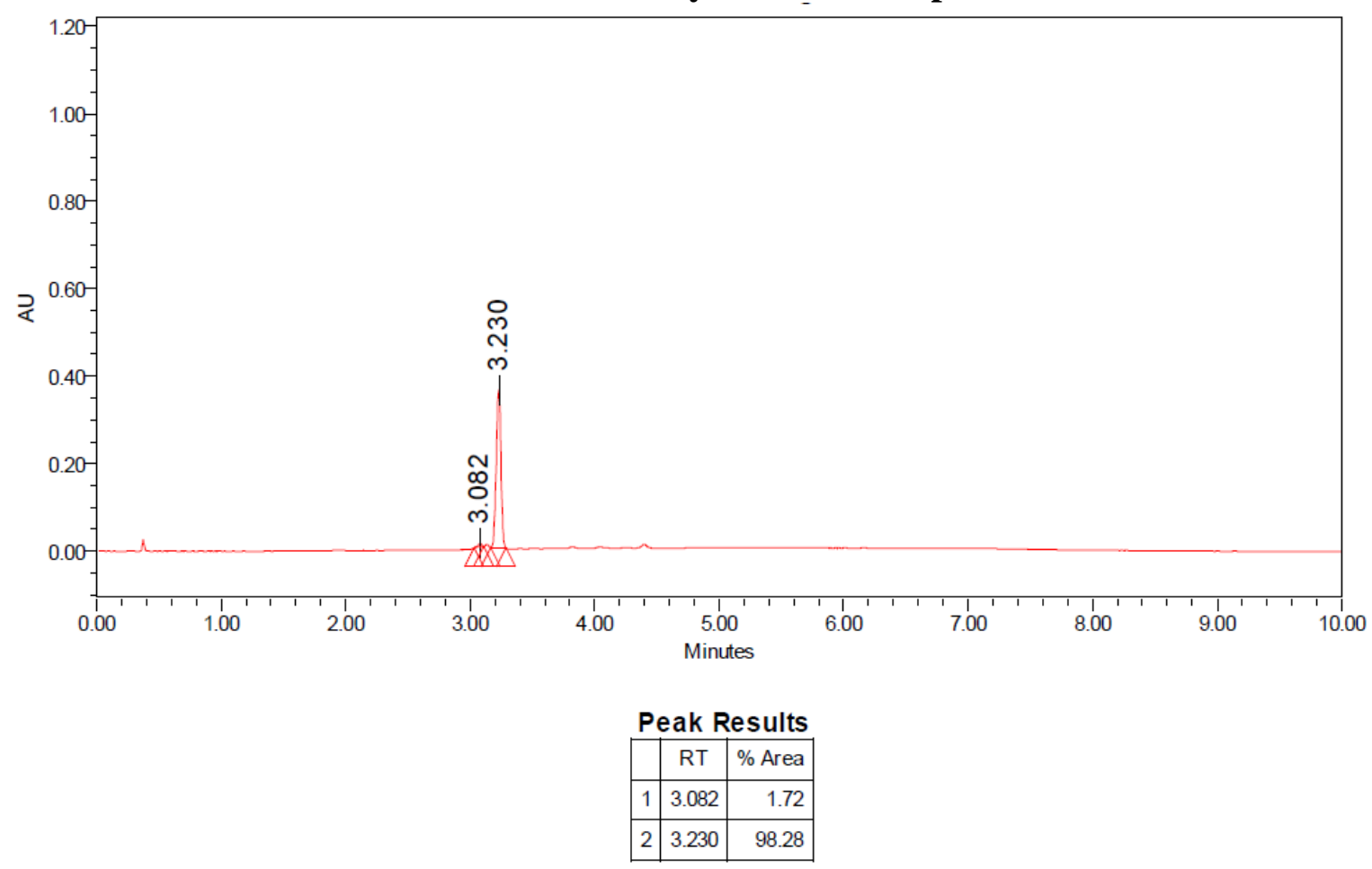


(6S,7S)-6-(2-Methoxyphenyl)-7-phenyl-6,7-dihydrobenzofuran-5-carbaldehyde 3g
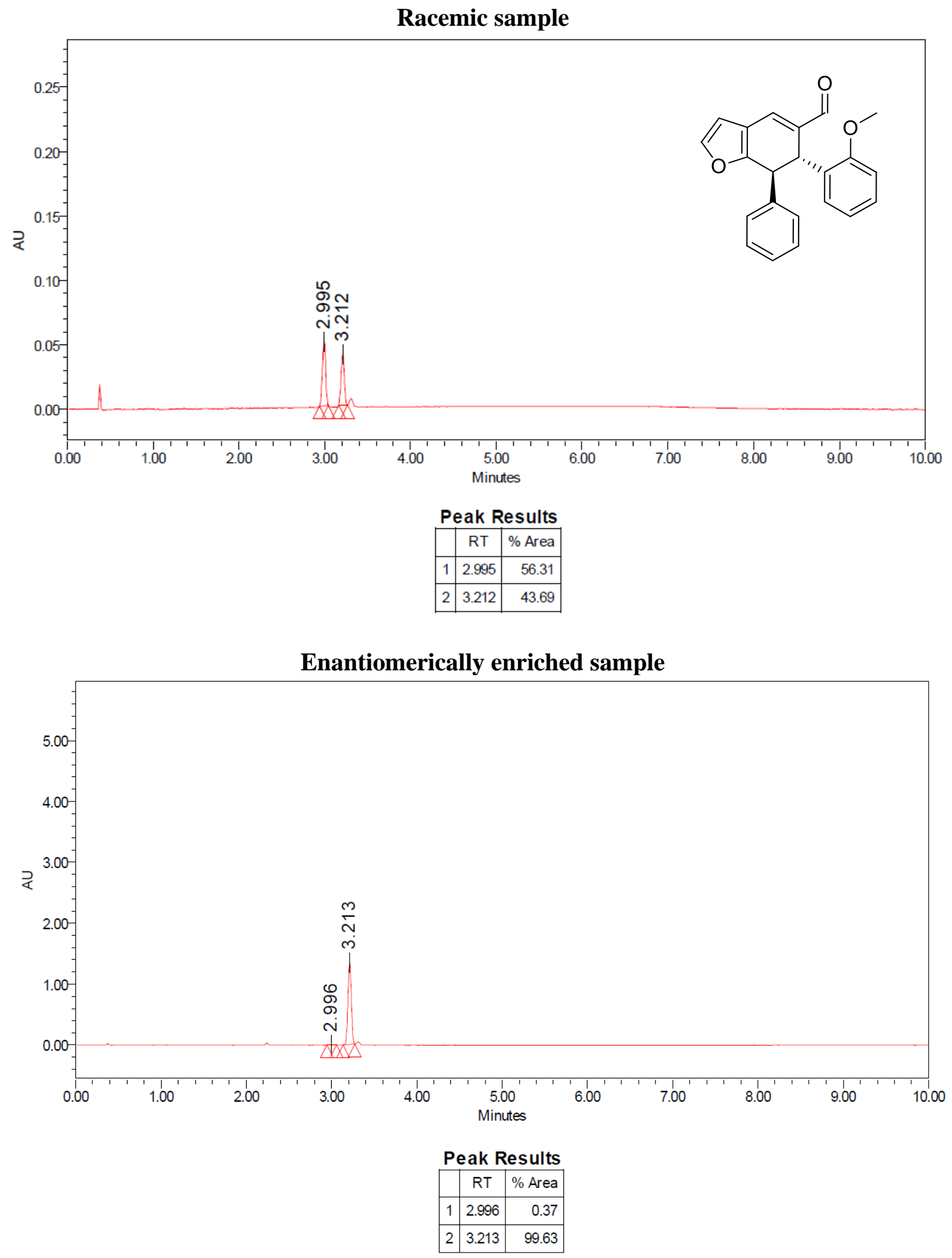
(6R,7S)-6-(2,4-Dichlorophenyl)-7-phenyl-6,7-dihydrobenzofuran-5-carbaldehyde $3 \mathrm{~h}$

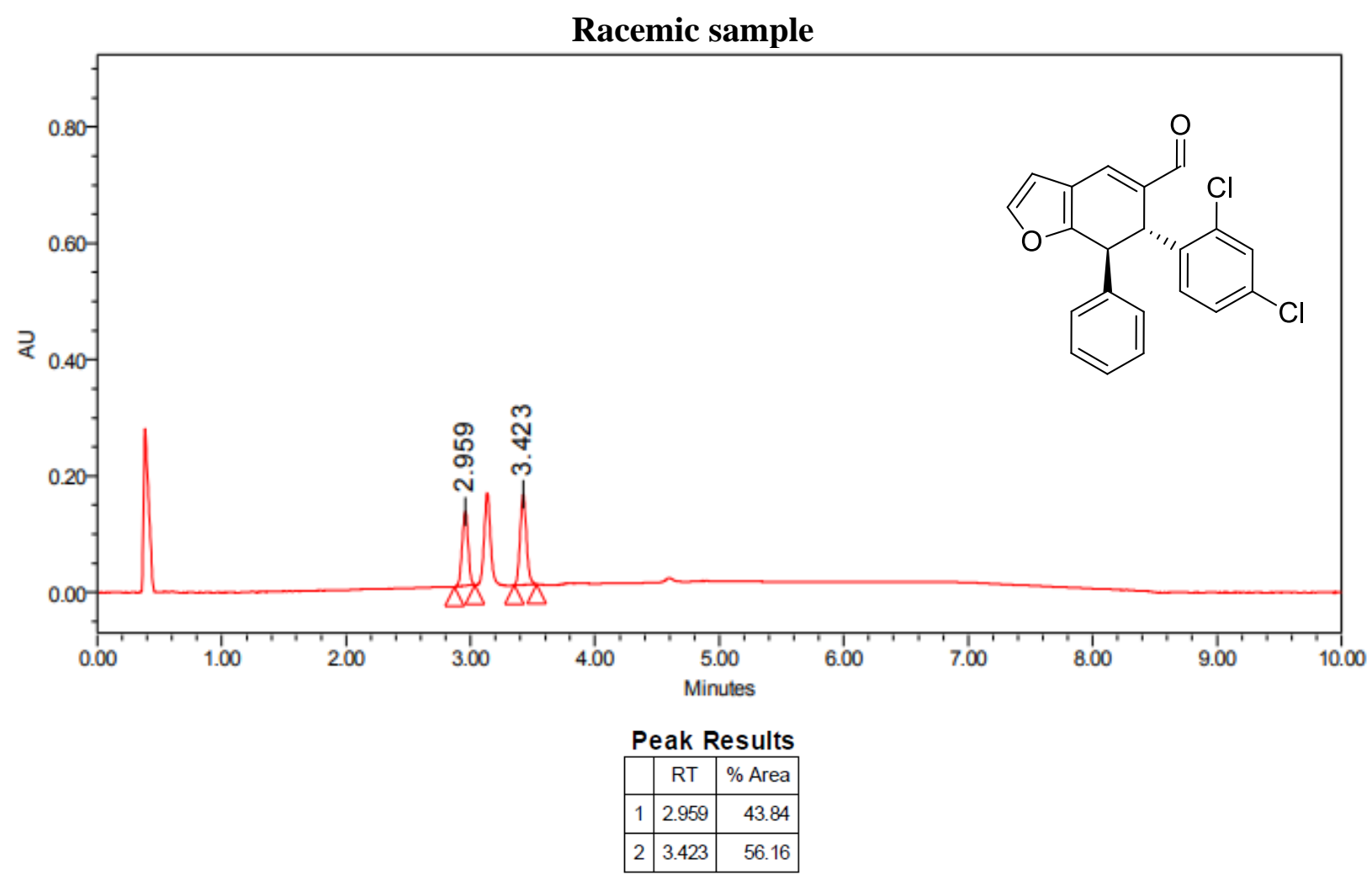

Enantiomerically enriched sample

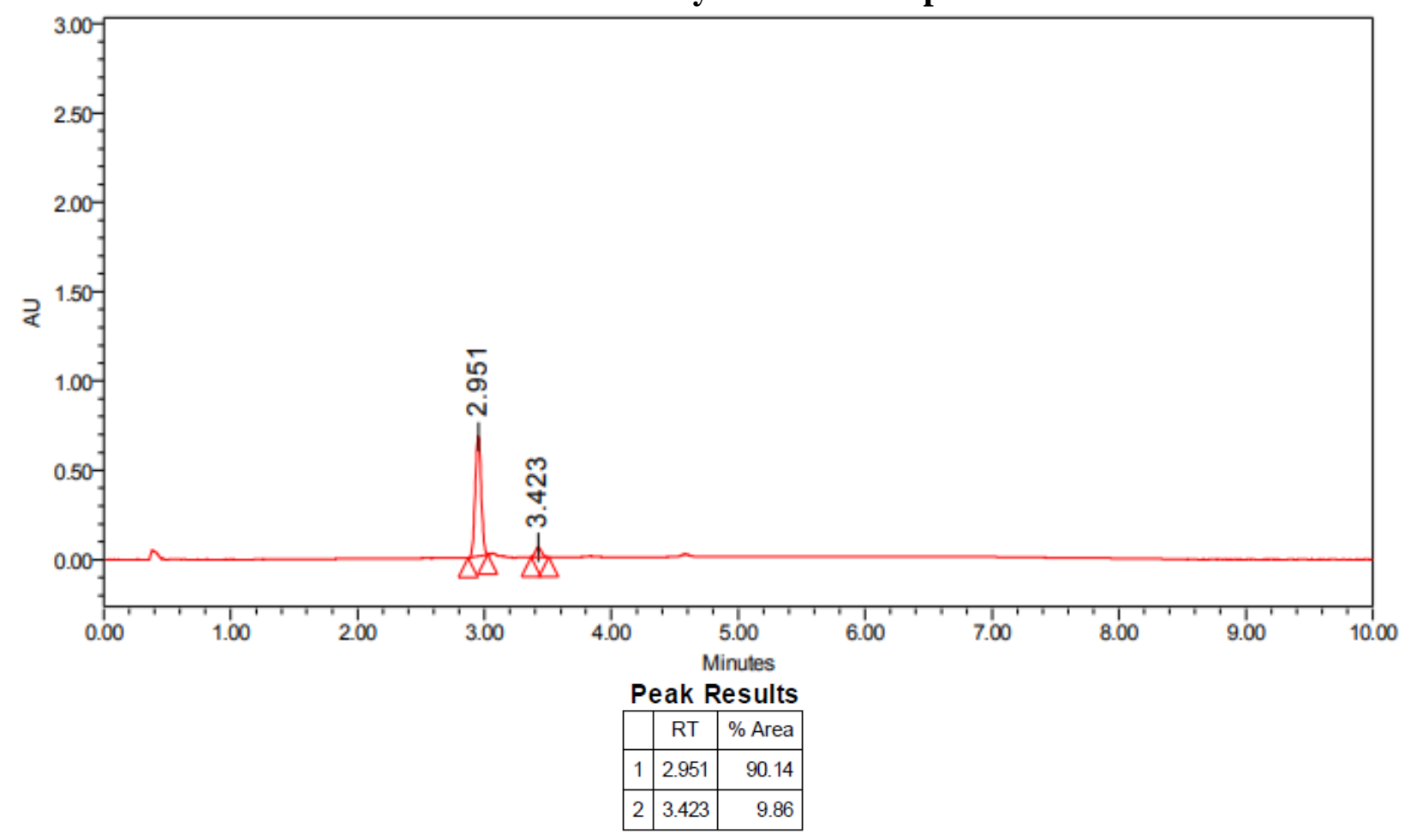


(6R,7S)-6-(Furan-2-yl)-7-phenyl-6,7-dihydrobenzofuran-5-carbaldehyde 3i

Racemic sample

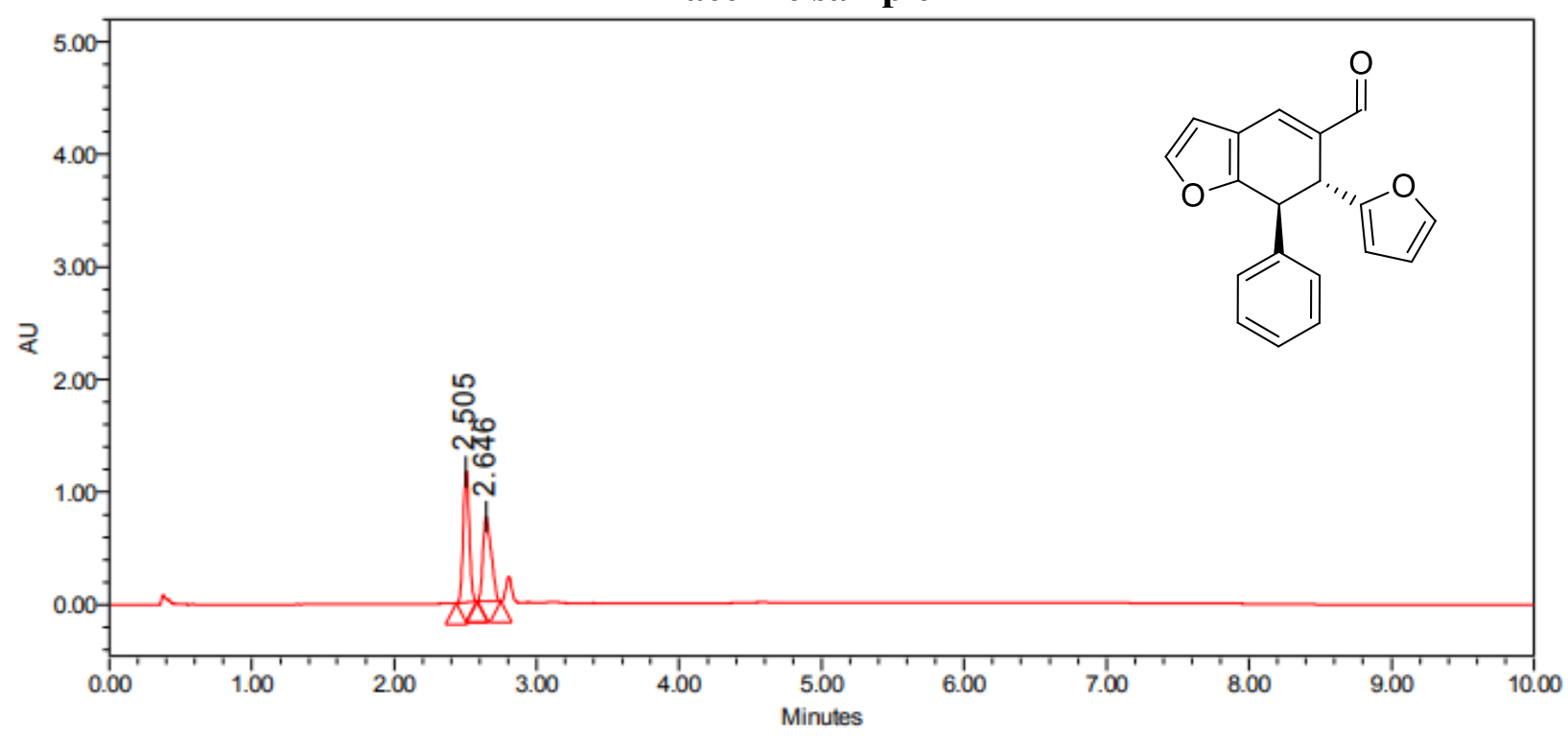

Peak Results

\begin{tabular}{|l|c|r|}
\hline & RT & \% Area \\
\hline 1 & 2.505 & 52.87 \\
\hline 2 & 2.646 & 47.13 \\
\hline
\end{tabular}

Enantiomerically enriched sample

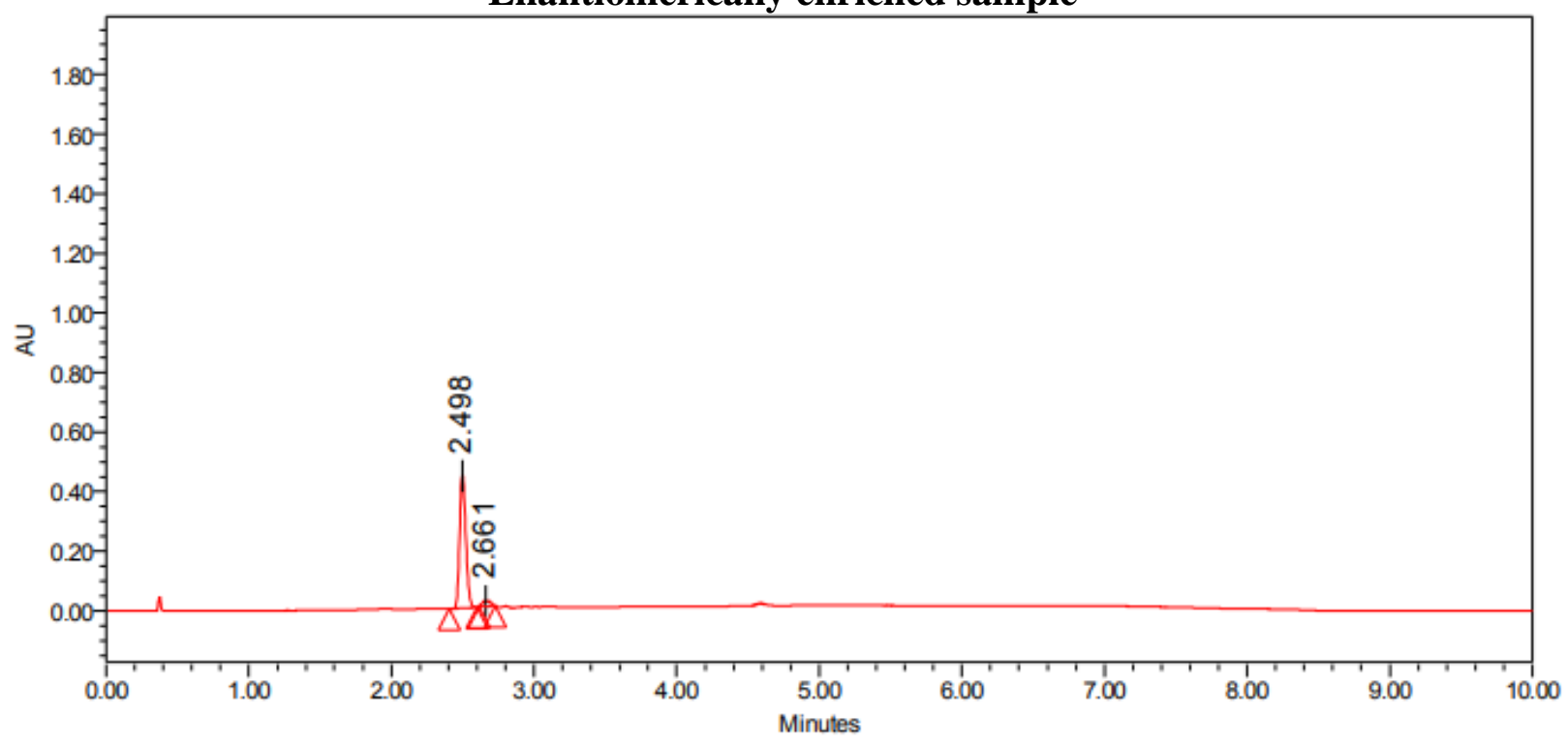

Peak Results
\begin{tabular}{|c|c|r|}
\hline & RT & $\%$ Area \\
\hline 1 & 2.498 & 94.68 \\
\hline 2 & 2.661 & 5.32 \\
\hline
\end{tabular}


(6S,7S)-7-(4-Fluorophenyl)-6-phenyl-6,7-dihydrobenzofuran-5-carbaldehyde 3j

Racemic sample

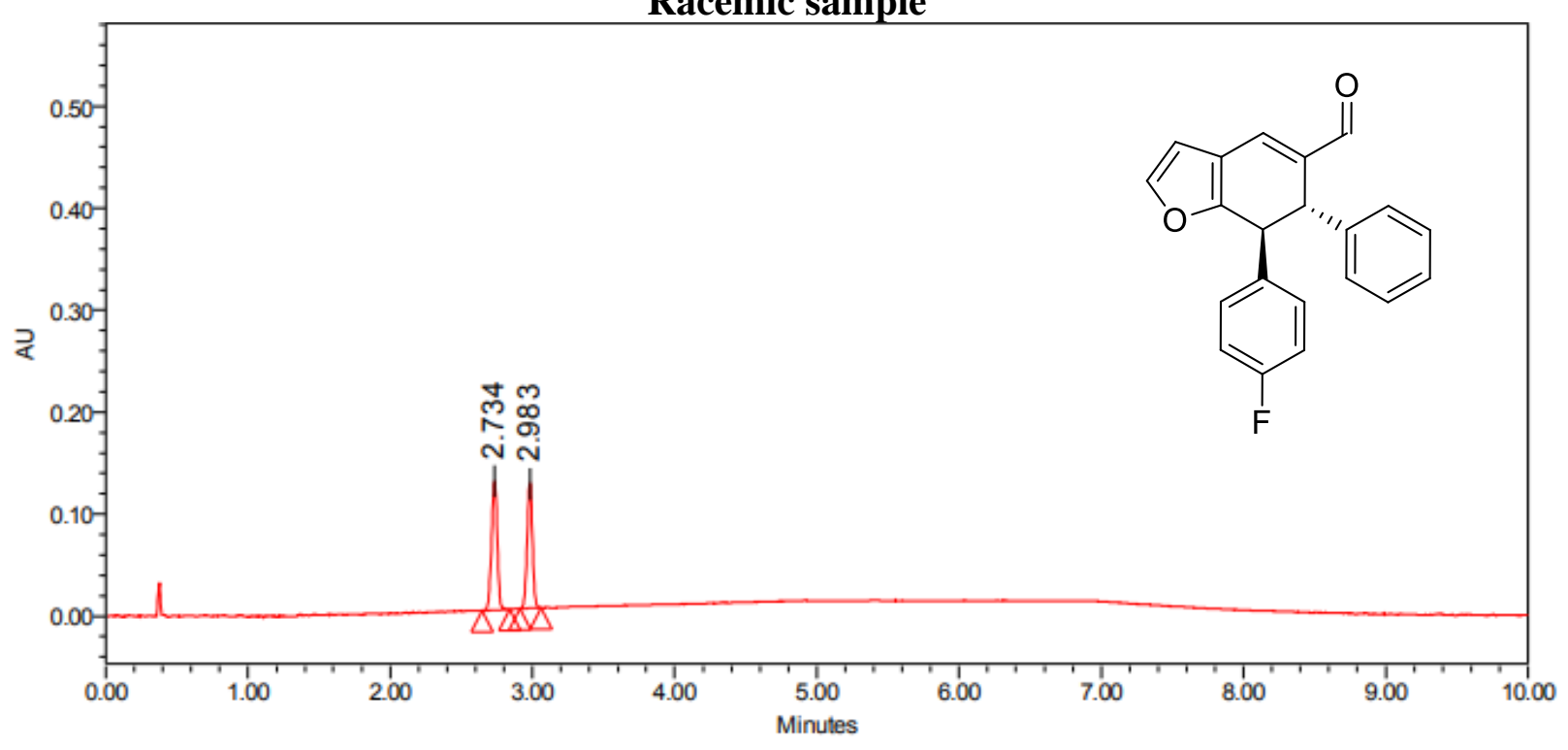

Peak Results

\begin{tabular}{|c|c|r|}
\hline & RT & \% Area \\
\hline 1 & 2.734 & 52.50 \\
\hline 2 & 2.983 & 47.50 \\
\hline
\end{tabular}

Enantiomerically enriched sample

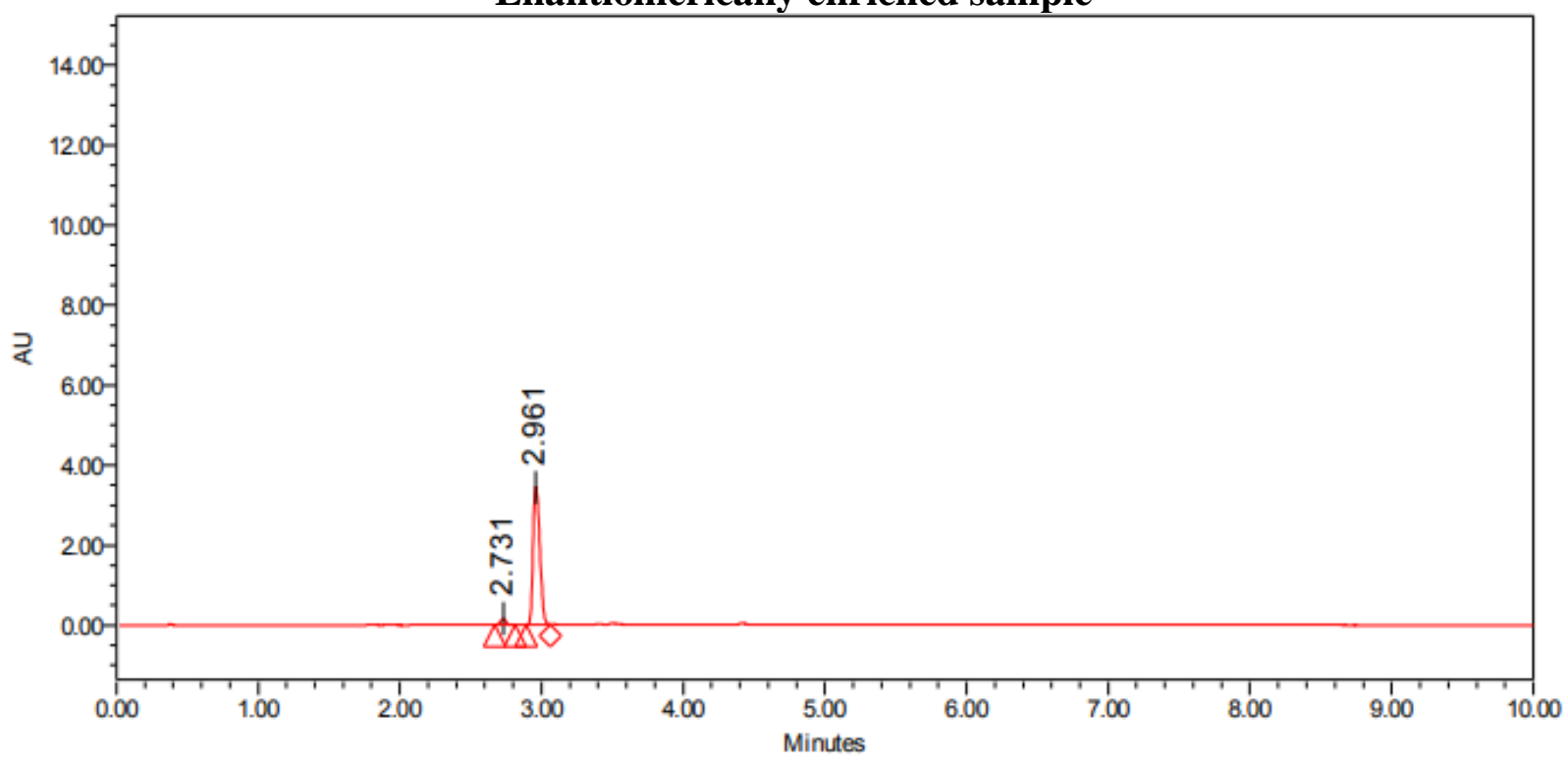

Peak Results

\begin{tabular}{|c|c|r|}
\hline & RT & \% Area \\
\hline 1 & 2.731 & 3.27 \\
\hline 2 & 2.961 & 96.73 \\
\hline
\end{tabular}


(6S,7S)-6-Phenyl-7-(p-tolyl)-6,7-dihydrobenzofuran-5-carbaldehyde 3k

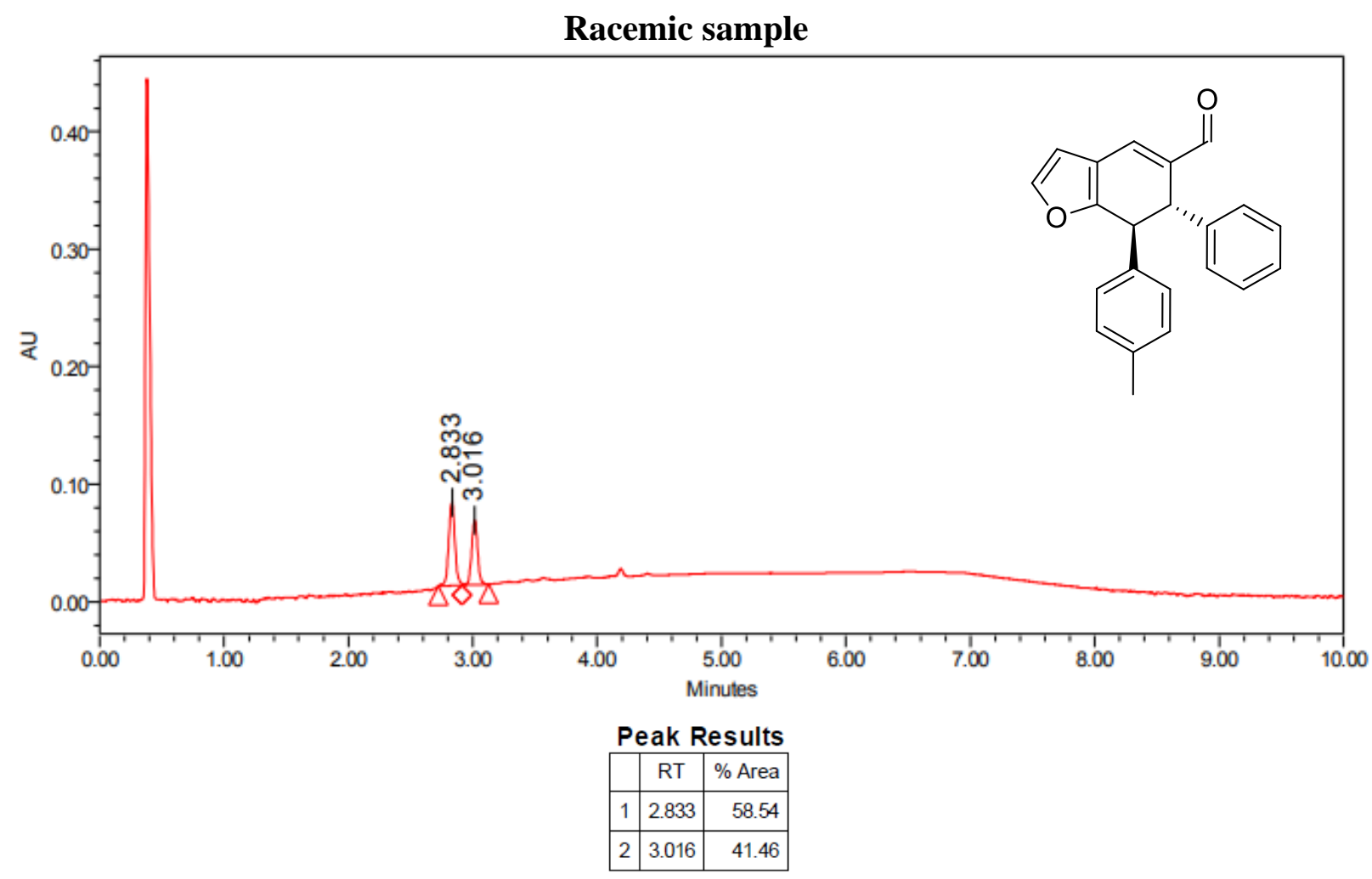

Enantiomerically enriched sample

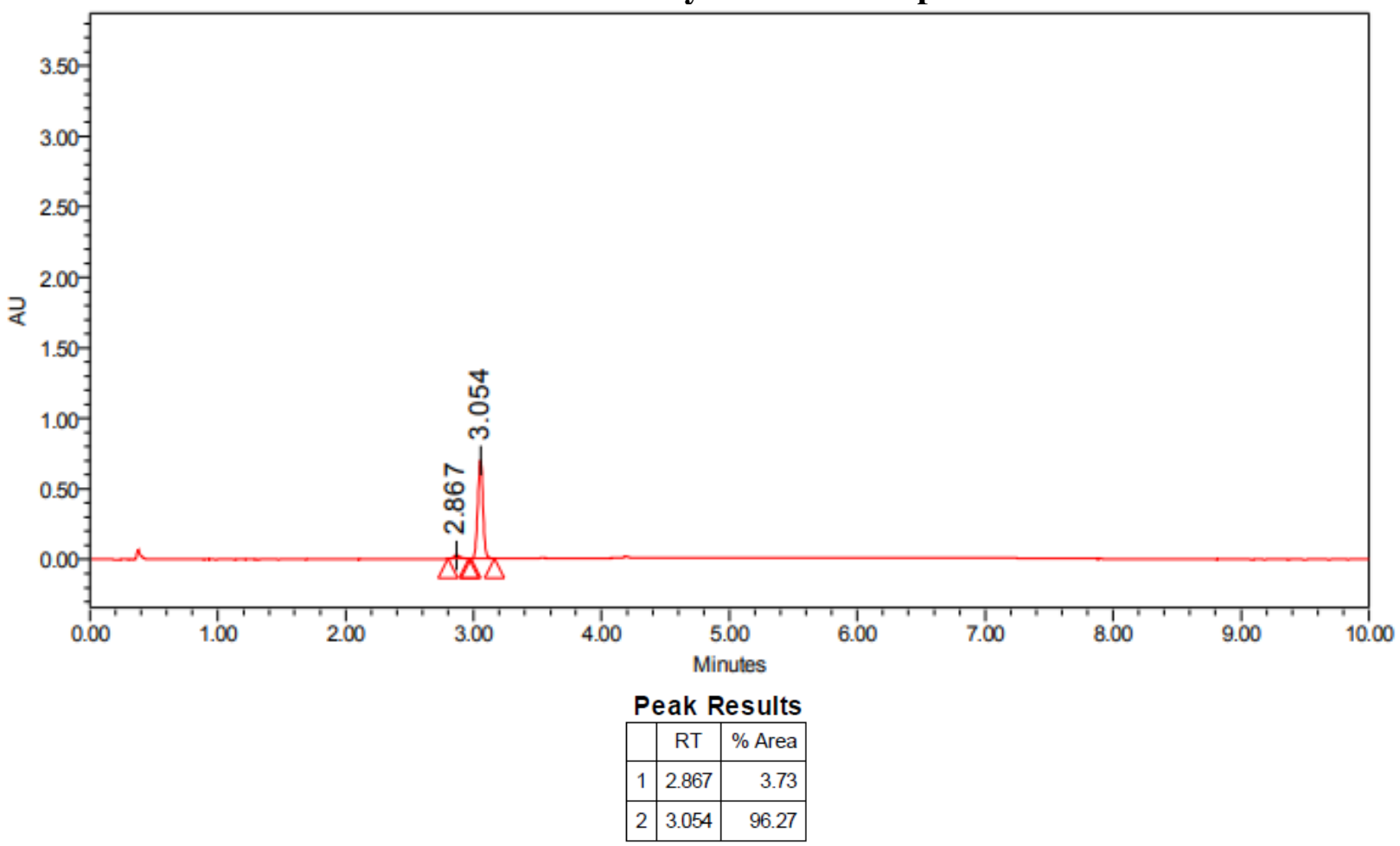


(6S,7S)-7-(3-Methoxyphenyl)-6-phenyl-6,7-dihydrobenzofuran-5-carbaldehyde 31

Racemic sample

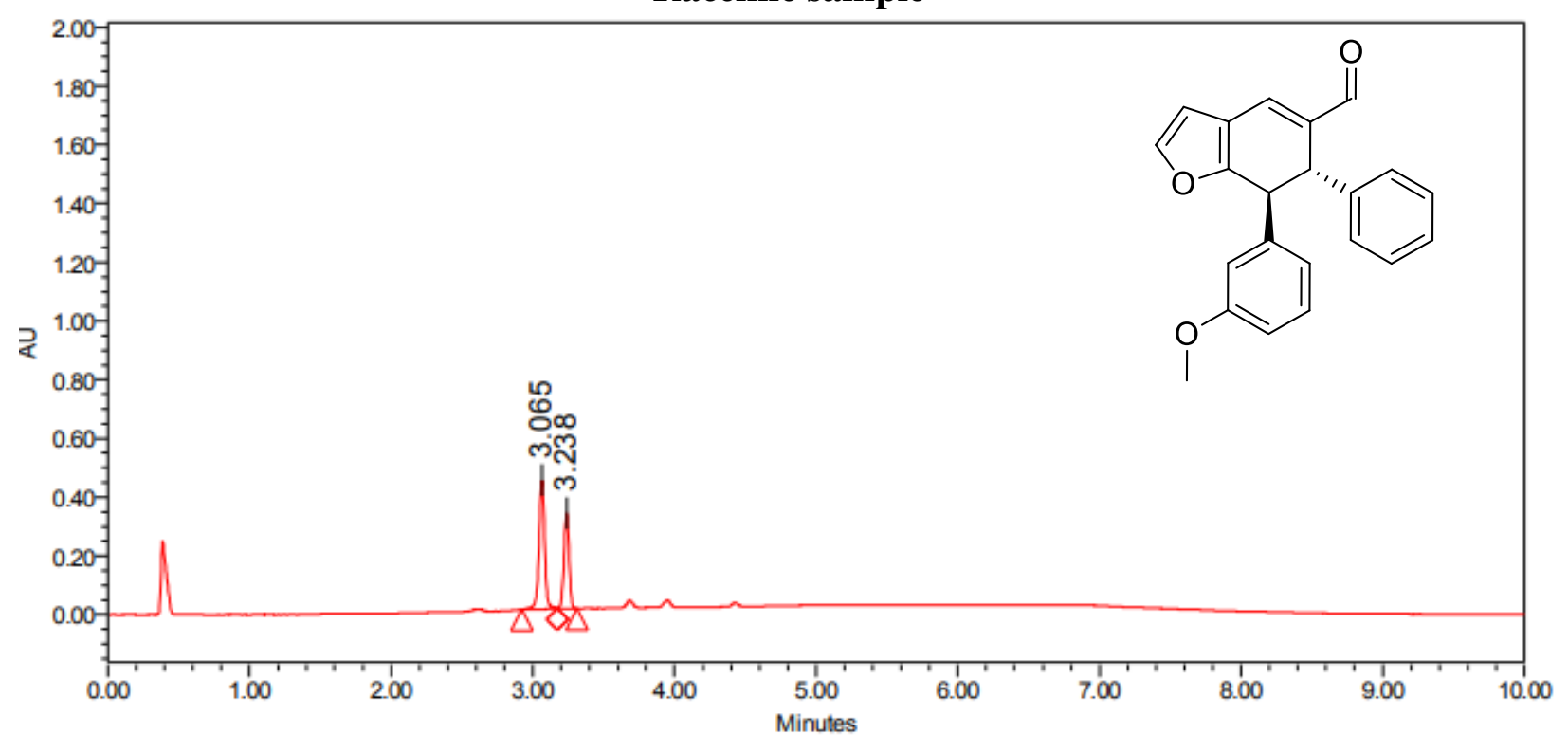

Peak Results
\begin{tabular}{|c|c|r|}
\hline & RT & $\%$ Area \\
\hline 1 & 3.065 & 60.42 \\
\hline 2 & 3.238 & 39.58 \\
\hline
\end{tabular}

Enantiomerically enriched sample

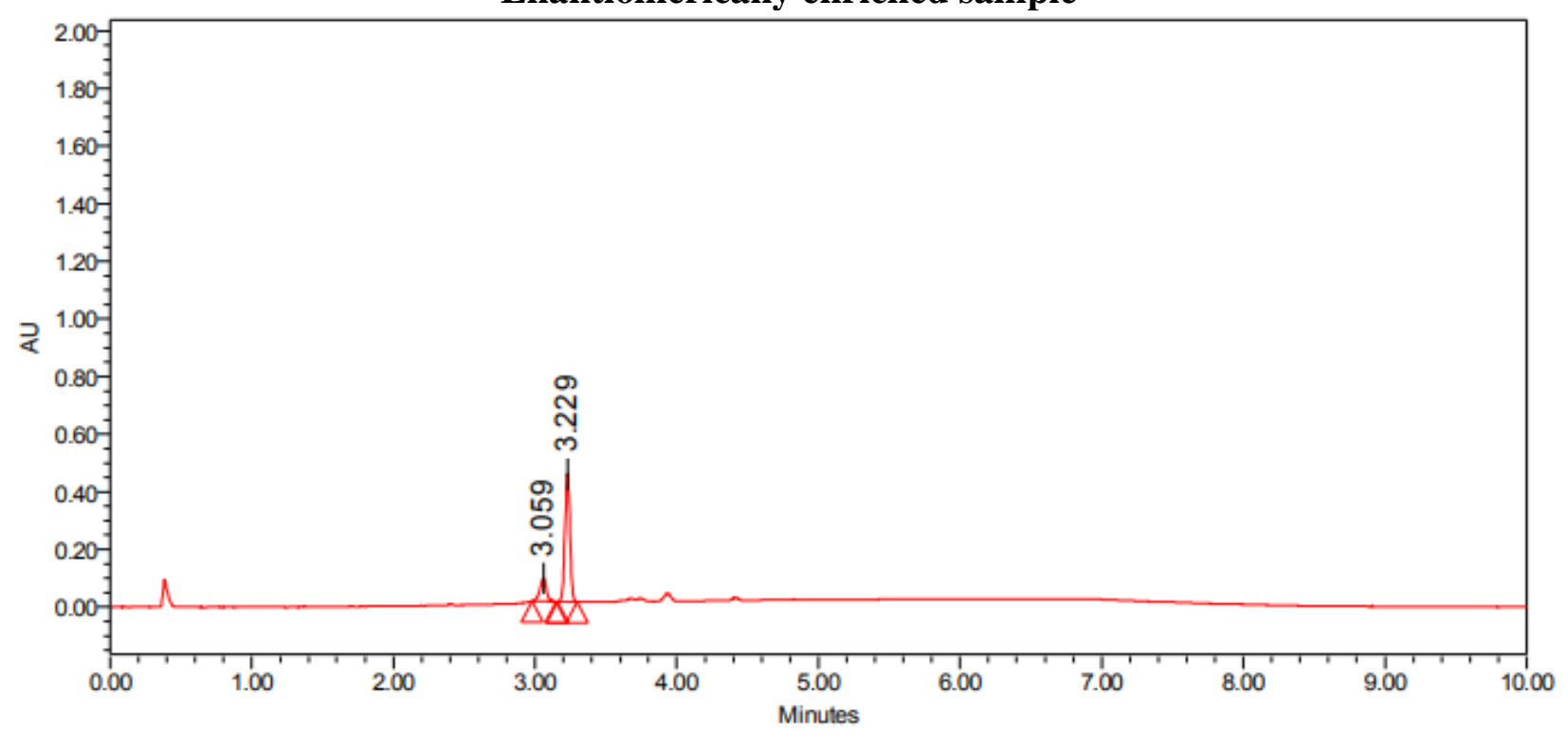

Peak Results

\begin{tabular}{|c|c|r|}
\hline & RT & \% Area \\
\hline 1 & 3.059 & 18.99 \\
\hline 2 & 3.229 & 81.01 \\
\hline
\end{tabular}


(6S,7S)-6-Phenyl-7-(0-tolyl)-6,7-dihydrobenzofuran-5-carbaldehyde 3m
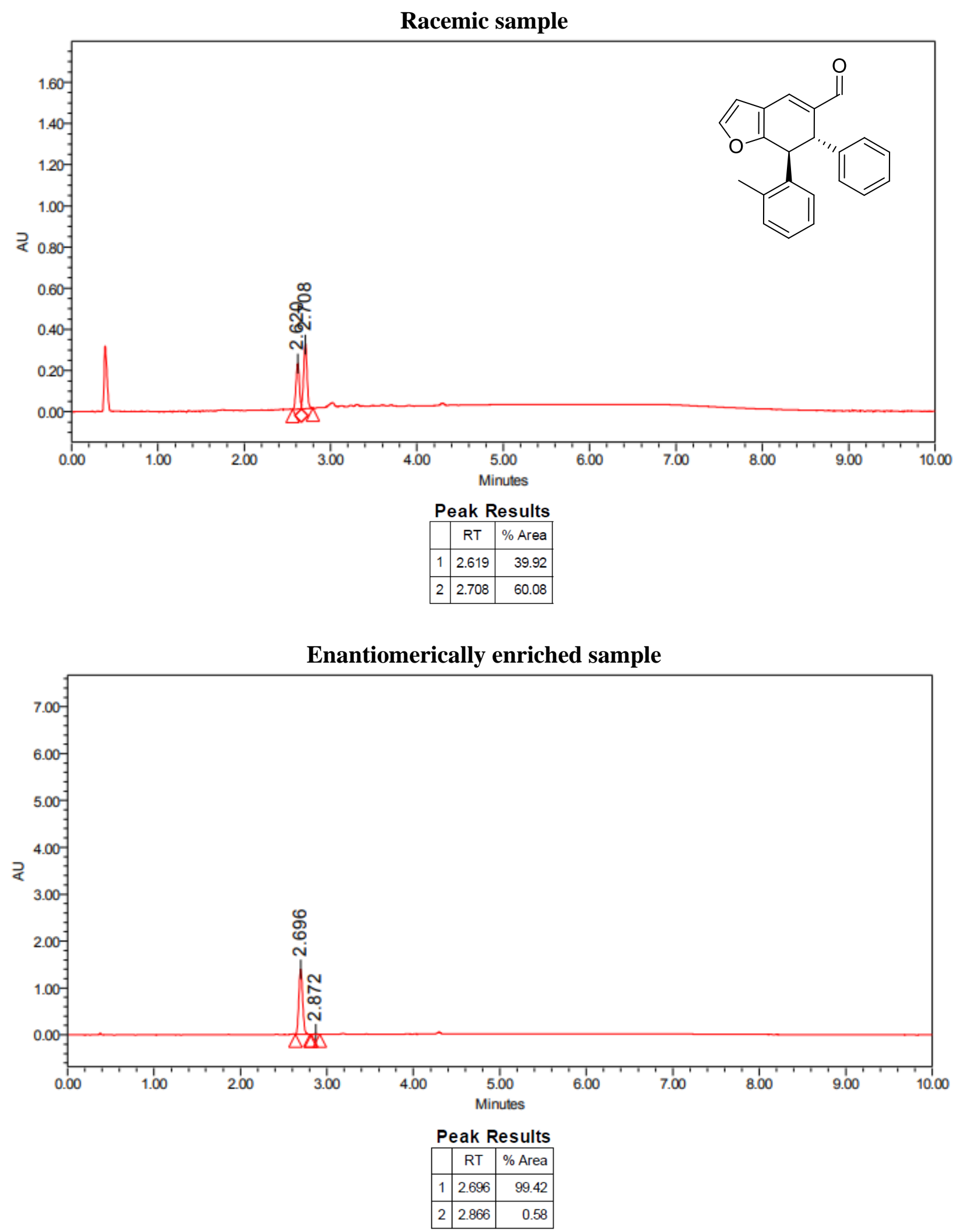
(6S,7R)-6-Phenyl-7-vinyl-6,7-dihydrobenzofuran-5-carbaldehyde 3n

Racemic sample

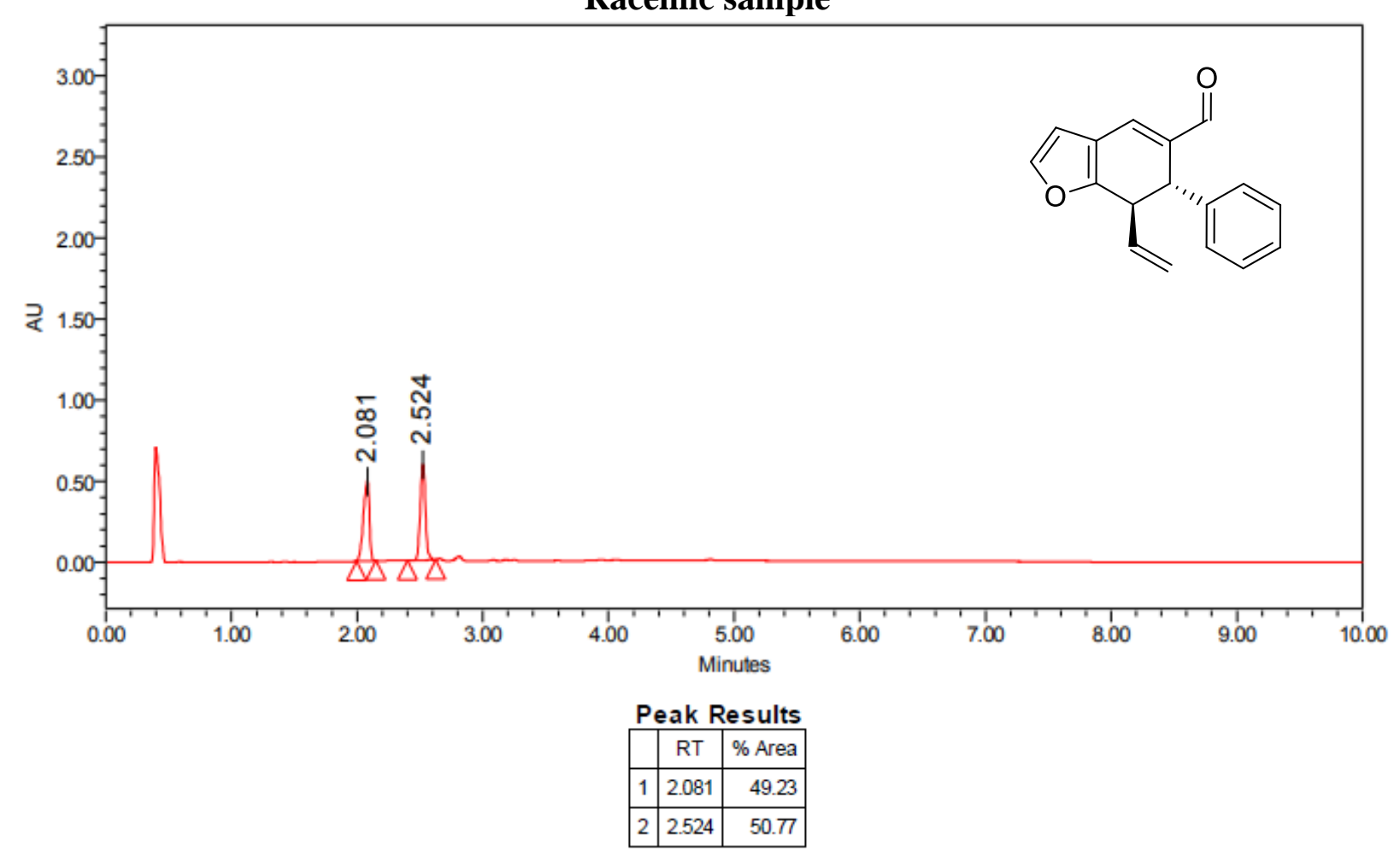

Enantiomerically enriched sample

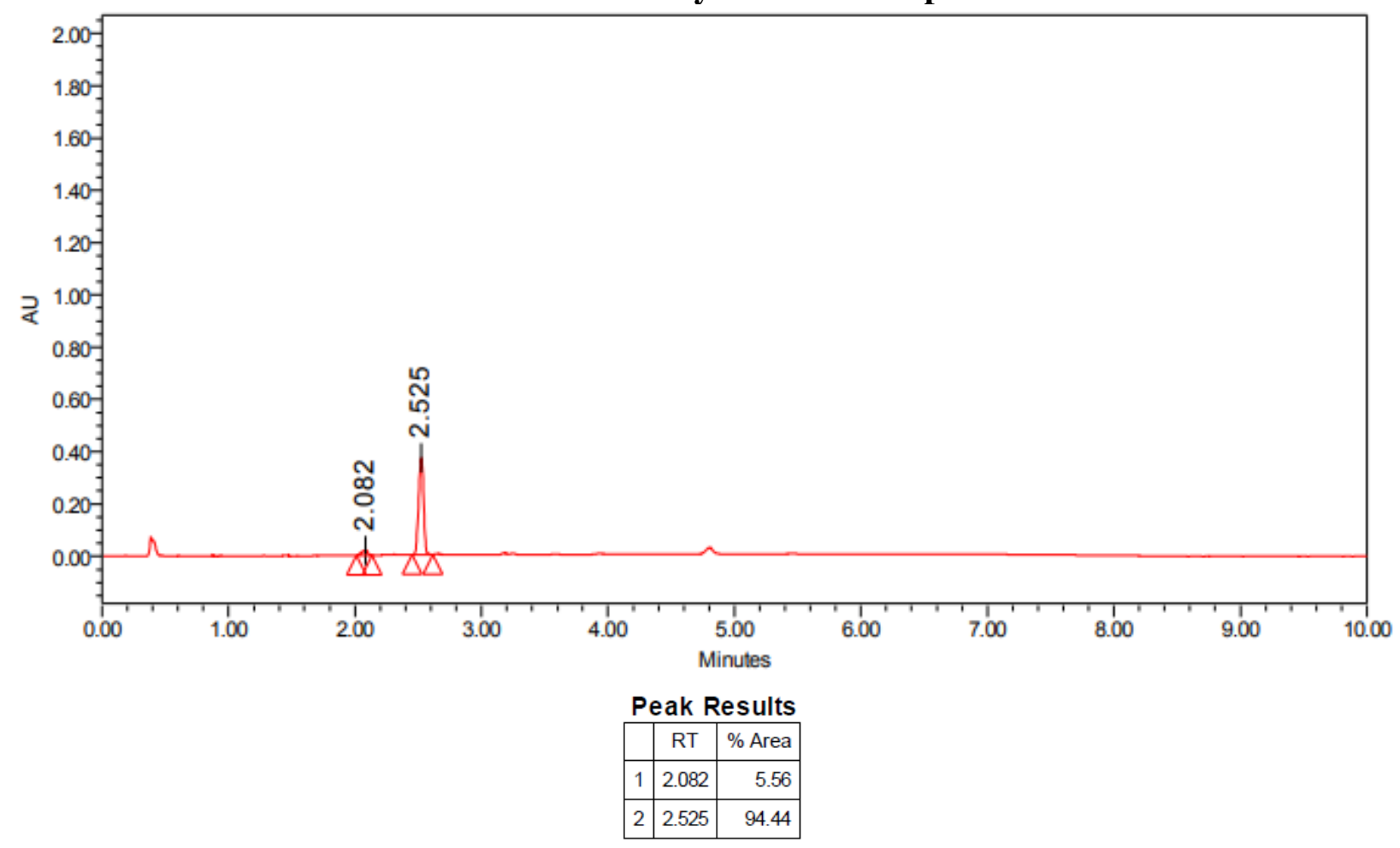




\section{(3S,4S)-3,4-Diphenyl-3,4-dihydrodibenzo[b,d]furan-2-carbaldehyde 30}

Racemic sample

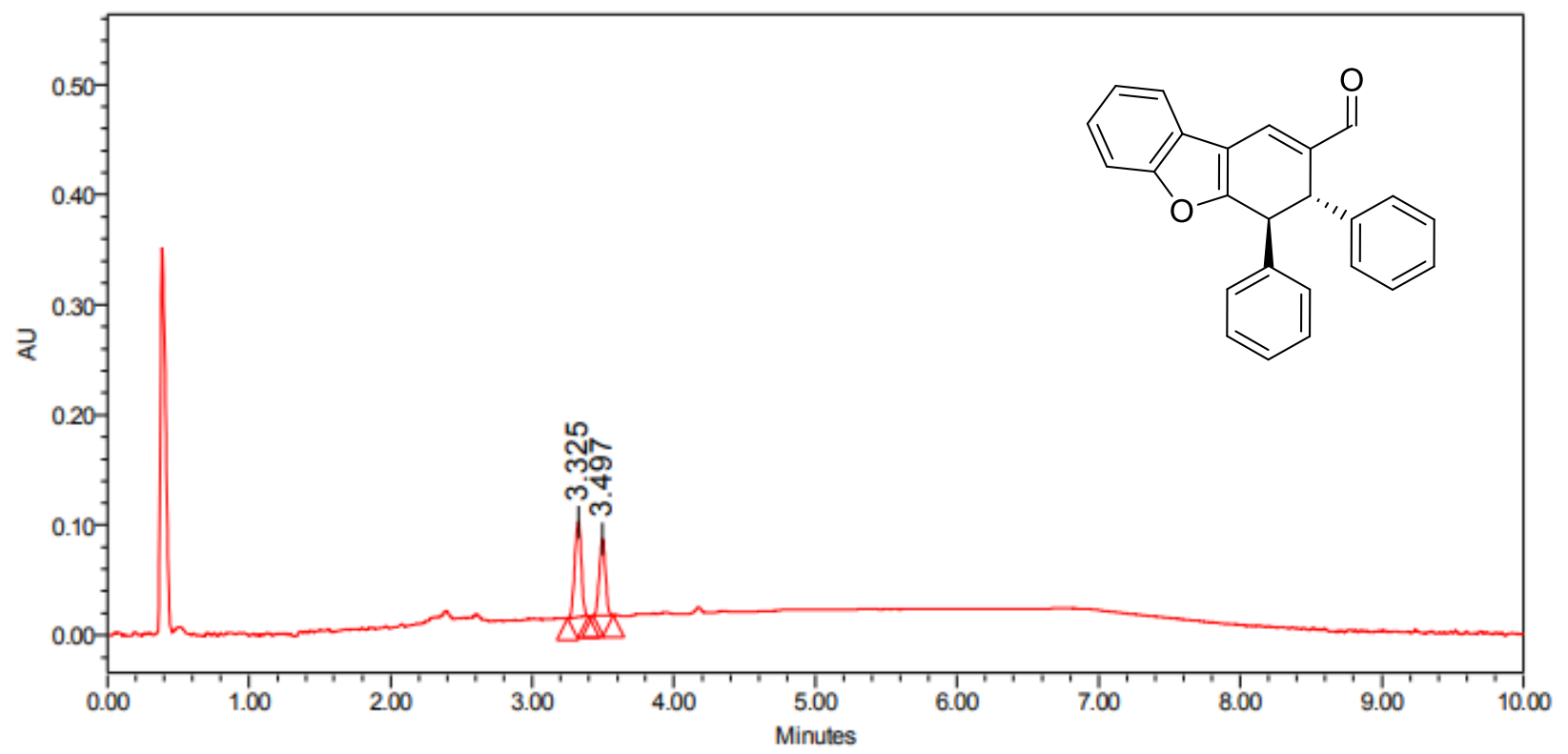

Peak Results

\begin{tabular}{|c|c|r|}
\hline & RT & \% Area \\
\hline 1 & 3.325 & 57.36 \\
\hline 2 & 3.497 & 42.64 \\
\hline
\end{tabular}

Enantiomerically enriched sample

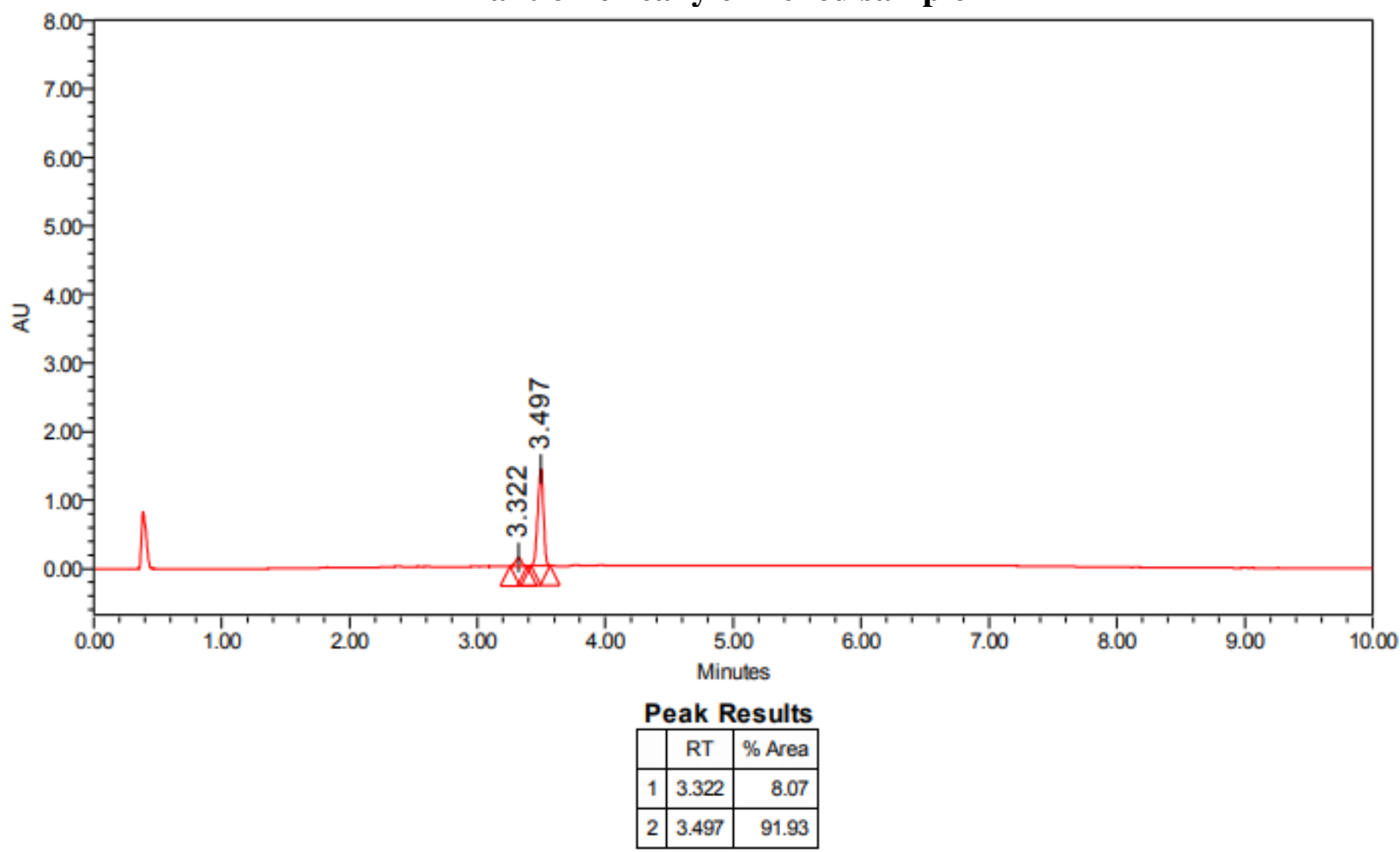

